\title{
The Role of Reculturalization in Adaptation: A Comparison of Humor Styles in the UK and U.S. Versions of "The Office"
}

\author{
Rachael A. McKinney
}

Follow this and additional works at: https://researchrepository.wvu.edu/etd

\section{Recommended Citation}

McKinney, Rachael A., "The Role of Reculturalization in Adaptation: A Comparison of Humor Styles in the UK and U.S. Versions of "The Office"'" (2016). Graduate Theses, Dissertations, and Problem Reports. 6201. https://researchrepository.wvu.edu/etd/6201

This Thesis is protected by copyright and/or related rights. It has been brought to you by the The Research Repository @ WVU with permission from the rights-holder(s). You are free to use this Thesis in any way that is permitted by the copyright and related rights legislation that applies to your use. For other uses you must obtain permission from the rights-holder(s) directly, unless additional rights are indicated by a Creative Commons license in the record and/ or on the work itself. This Thesis has been accepted for inclusion in WVU Graduate Theses, Dissertations, and Problem Reports collection by an authorized administrator of The Research Repository @ WVU. For more information, please contact researchrepository@mail.wvu.edu. 


\title{
Bee Natural History, Diversity, and Management in West Virginia
}

\author{
Matthew McKinney
}

Dissertation submitted

to the Davis College of Agriculture, Natural Resources, and Design at West Virginia University

in partial fulfillment of the requirements for the degree of

Doctor of Philosophy in

Entomology

\author{
Yong-Lak Park, Ph.D., Chair \\ Zachary Loughman, Ph.D. \\ Michael Strager, Ph.D. \\ David McGill, Ph.D. \\ Daniel Frank, Ph.D.
}

Division of Plant and Soil Sciences

Morgantown, West Virginia 2016

Keywords: Hymenoptera, Apoidea, Bees, West Virginia, Sampling, Osmia, Monodontomerus, Bee Bowls, Pan Traps, Temperature Dependent Development

Copyright 2016 Matthew McKinney 


\section{Abstract \\ Bee Natural History, Diversity, and Management in West Virginia}

\section{Matthew McKinney}

The value of bees as providers of pollination, an incredibly important ecosystem service, is well understood. Since the detection of colony collapse disorder, the concern over the health of both managed and naturally occurring bee populations has been in the limelight, sometimes being discussed nearly as much in popular media as it is in scientific research. While an ideal situation may be the complete recovery of Apis mellifera populations, the causes of colony losses are not fully understood. Therefore, we must also consider our alternative options such as managing areas to better support natural bees and utilizing alternative managed species, such as Osmia cornifrons, in the most effective way possible. The goals of this research were (1) to determine the faunal diversity of bees in West Virginia and to enhance monitoring programs for future survey efforts, and (2) to elucidate some problems that may arise when utilizing those species of bees which are commonly managed as alternative pollinators in West Virginia. The results of this study showed that there are 301 currently recognized bee species within the boundaries of West Virginia, and there are likely many more not yet discovered due to lack of collecting effort in some areas of the state. To achieve independent samples when utilizing pan traps for survey work, a distance of $18 \mathrm{~m}$ between traps is necessary. Osmia cornifrons was found to be more sensitive to temperature than many other insects, including other bees, and male bees were found to be at greater risk of parasitism by Monodontomerus spp. then females. This study provides fundamental and useful information for the conservation and management of native and managed bee species in West Virginia. 


\section{Acknowledgements}

I would like to thank West Virginia University for giving me the opportunity to pursue my goals as a researcher and educator. I would like to thank my committee members for their helpful guidance and support throughout the process of completing this work. I would also like to thank Dr. Patrick Tobin for serving on my committee for the short time he was able to do so.

I would like to express my gratitude to Dr. Zachary Loughman, who inspired me as a young student of the biological sciences to continue onward and seek greater things, and who offered me words of wisdom when I needed them most. I would like to express my utmost appreciation to Dr. Yong-Lak Park, my committee chair, for expending such tremendous effort on my behalf, for his infinite patience, and for opening my eyes to ideas I would not have considered.

It has been my privilege to collaborate and discuss scientific ideas with many people, and while there are too many to list here, I appreciate all of their help and support. I thank Dr. Jeong-Joon Ahn for his analytical contributions to this work. Sam Droege from the USGS must be thanked for the tremendous amount of data he sent me for the checklist of the bees of West Virginia. There would be a far smaller list without the effort he has made collecting and identifying bees from the eastern portion of the state. I must also thank Ansel Payne and John Ascher, the two most patient collaborators anyone could ask for, for their numerous contributions to checklist of West Virginia bees.

I must thank my mother and father, Yvonne and Isaac McKinney for their support throughout these many years.

Finally, I express my deepest love and gratitude to my wife Kayla, who has shared these challenges with me, and who has picked me up when I've fallen down. Without her love and friendship I would truly be lost. 


\section{TABLE OF CONTENTS}

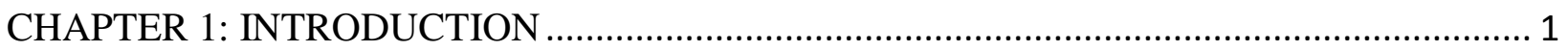

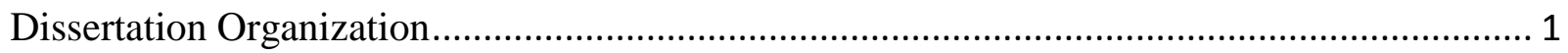

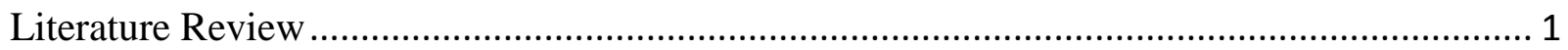

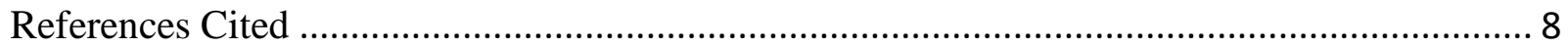

CHAPTER 2: CHECKLIST OF THE BEES OF WEST VIRGINIA .................................... 13

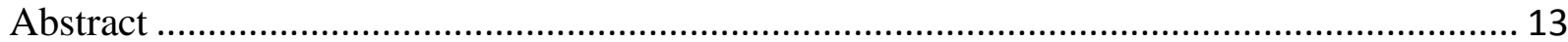

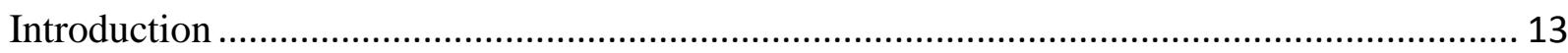

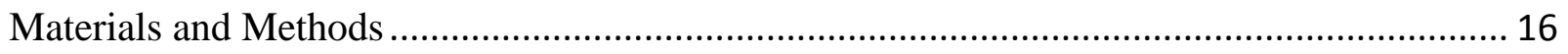

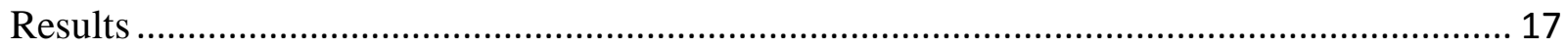

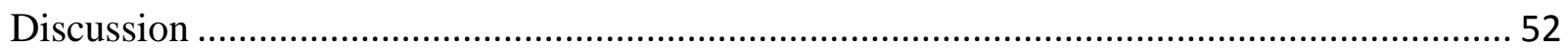

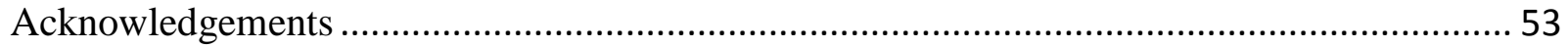

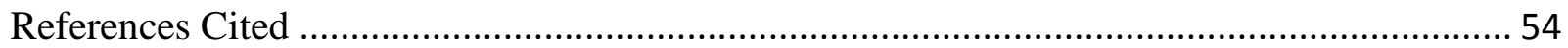

CHAPTER 3: DETERMINATION OF SPATIAL DEPENDENCE AMONGST PAN TRAPS IN

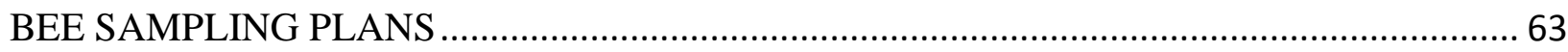

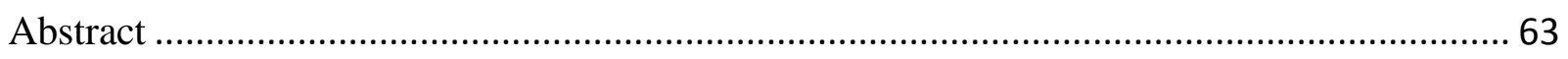

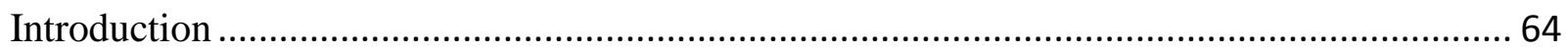

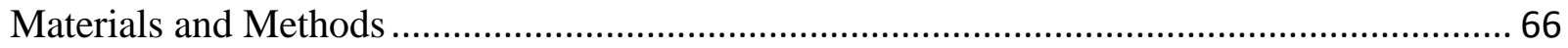

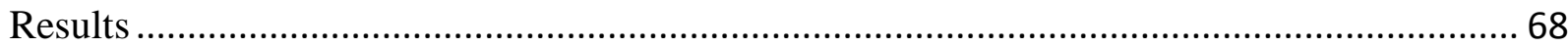

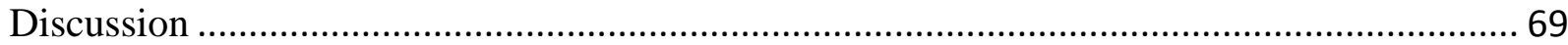

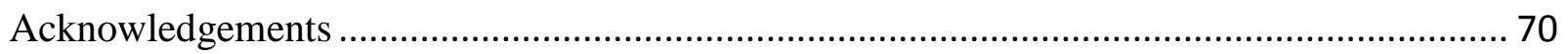

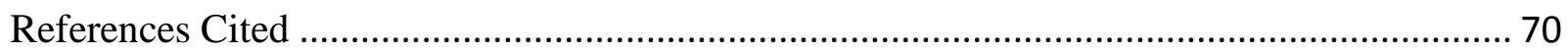

CHAPTER 4: THERMAL BIOLOGY OF Osmia cornifrons (HYMENOPTERA:

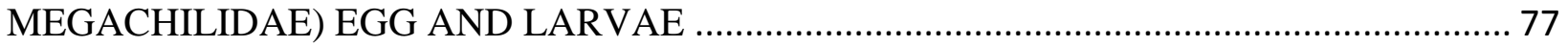

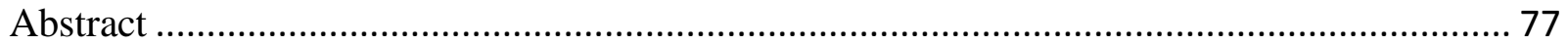

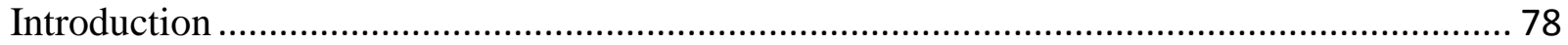

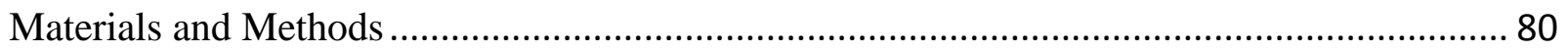

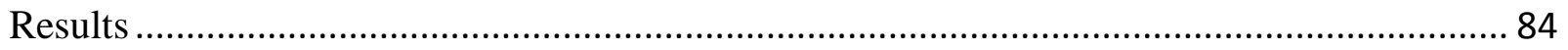

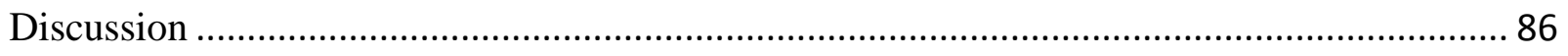

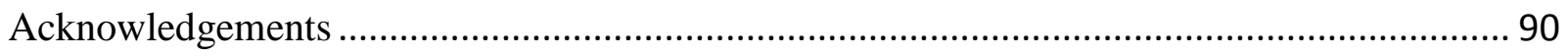

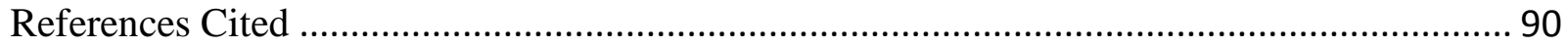


CHAPTER 5: SPATIAL TRENDS OF Monodontomerus WESTWOOD (HYMENOPTERA: TORYMIDAE) IN NESTS OF Osmia cornifrons (HYMENOPTERA: MEGACHILIDAE) ... 102

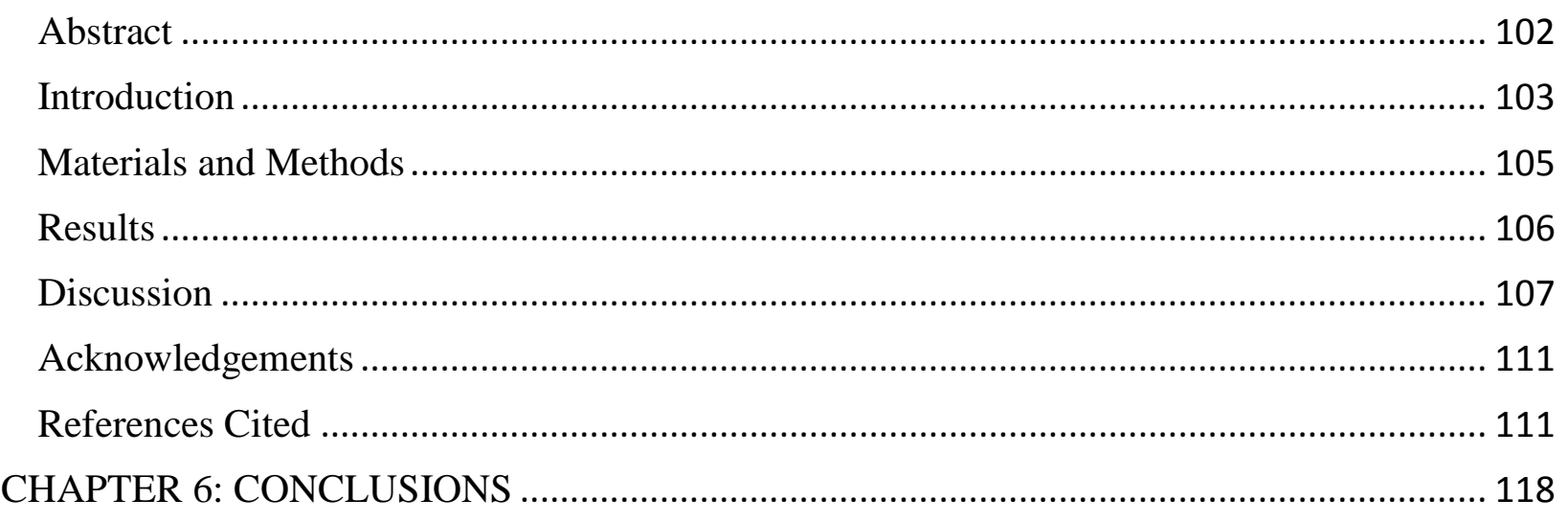




\section{LIST OF TABLES}

\section{Chapter 2}

Table 1. Museum collection codes used in the species list.

Table 2. List of problematic taxa. The species listed below could not be confirmed as part of the West Virginia fauna. These include: 1) published records based on misidentifications, 2) unverifiable identifications, and 3) accidental occurrences.

\section{Chapter 3}

Table 1. Semivariogram model parameters* for four study sites........................................................73

Table 2. Bee genera found at each of the four study sites................................................................ 74

Table 3. Results of chi-square test using the hypothesis that all bee genera would be found in equal

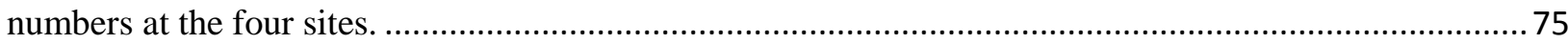

\section{Chapter 4}

Table 1. Mean developmental times (days) (mean \pm SEM) and survivorship (\%) of Osmia cornifrons at

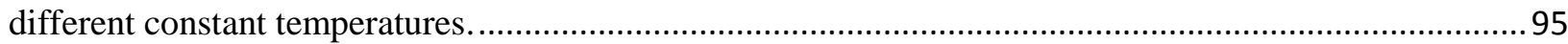

Table 2. Parameter estimates $( \pm$ SEM) for nonlinear developmental rate model (Briére 1 model) for Osmia

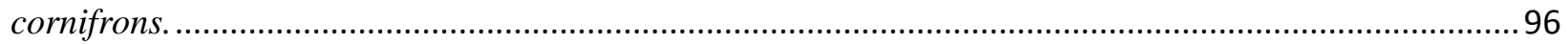

Table 3. Estimated parameters of Weibull function for Osmia cornifrons. ............................................ 97

\section{Chapter 5}

Table 1. Anisotropic semivariogram parameters for the distribution of Monodontomerus spp. emergence holes in Osmia cornifrons nest bundles. 


\section{LIST OF FIGURES}

\section{Chapter 2}

Figure 1. Collecting effort throughout West Virginia depicted as the percentage of collecting trips made in each county.

Figure 2. Pie charts showing percentage of specimens from each family included in the list (A) and percentage of species recorded from each family (B).

\section{Chapter 3}

Figure 1. Example of experimental plot. Large points indicate the location of a pair of pan traps; one painted fluorescent yellow and the other fluorescent blue. Small points represent pairs of pan traps placed at smaller distance intervals to detect small scale spatial autocorrelation.

\section{Chapter 4}

Figure 1. Relationships between temperature and developmental rates $\left(\right.$ day $\left.^{-1}\right)$ of Osmia cornifrons: A. Egg to larva, B. Larva to prepupa, C. Egg to prepupa. Linear regression of the egg stage is $\mathrm{rT}=$ $0.0600 \mathrm{~T}-0.7646$ with the lower developmental threshold of $12.73^{\circ} \mathrm{C}$ and thermal constant of 16.66 degree-days (DD).

Figure 2. Observed cumulative proportions of Osmia cornifrons developed at normalized developmental time (i.e. developmental time / mean developmental time) fit with the two-parameter Weibull function: A. Egg to larva, B. Larva to prepupa, C. Egg to prepupa.

Figure 3. Validation of temperature-dependent development model for egg to prepupal stage of Osmia cornifrons by using field data collected from two locations (Morgantown and Kearneysville) in West Virginia.

Figure 4. Simulated frequency of temperature-dependent development of Osmia cornifrons: A. Egg to larva, B. Larva to prepupa, and C. Egg to prepupa.

\section{Chapter 5}

Figure 1. Distribution of Osmia cornifrons (A) and Monodontomerus (B) shows male O. Cornifrons may be more susceptible than females to wasp parasitism.

Figure 2. Anisotropic semivariograms for Morgantown (A) and Kearneysville (B) showing the linear relationship between semivariance and lag distance of Monodontomerus parasitism in cardboard straw bundles.

Figure 3. Interpolation of Monodontomerus spp. emergence holes in nests of Osmia cornifrons generated by using kriging. 


\section{CHAPTER 1: INTRODUCTION}

\section{Dissertation Organization}

This dissertation is organized into six chapters. Chapter 1 provides a general introduction to the study and a literature review. Chapter 2 describes the bee (Hymenoptera: Apoidea) fauna of West Virginia known from both recent and historical collections. Chapter 3 investigates the collecting of independent samples when using pan traps in bee survey and monitoring programs. Chapter 4 details the thermal biology of Osmia cornifrons (Hymenoptera: Megachilidae) with considerations regarding climate change and management. Chapter 5 elucidates spatial patterns of the parasitic wasp Monodontomerus spp. (Hymenoptera: Torymidae) as they are found in nests of $O$. cornifrons. Chapter 6 provides general conclusions drawn from this study. This dissertation was prepared according to the publication guidelines established by the Entomological Society of America with the exception of chapter 4, which was prepared following guidelines for the Journal of Apicultural Research.

\section{Literature Review}

\section{Importance of Bees}

To understand the importance of bees, one must first understand the importance of pollination. Pollination is a co-evolved process between animals and flowering plants in which pollen grains from a plant are transferred to the gynoecium (carpels) of another plant of the same species (Abrol 2012). The process of pollination is not strictly animal mediated, but that is the most common pollination strategy. Pollination is an essential ecosystem service, being economically valuable to humans, essential to the production of crops (Klein et al. 2007) and the 
reproduction of non-agricultural plants, and of tremendous importance to wildlife as a source of food. Globally, thousands of bee species visit plant crops (Free 1993), pollinating an estimated $73 \%$ of crops which require pollination (Nabhan and Buchmann 1997). Furthermore, Morse and Calderone (2000) estimated the value of pollination from honeybees to be $\$ 14.6$ billion annually in the U.S. Estimates in 2003 were as high as $\$ 27$ billion, and it is estimated that at least $15 \%$ of that number comes from native (non-honeybee) pollinators (Mader et al. 2011).

There are numerous pollinators worldwide, from the miniscule thrips (Insecta: Thysanoptera) to the charismatic butterfly (Insecta: Hymenoptera); even lemurs (Primates: Stepsirrhini) lend a hand in this vital task. Of all of the pollinators, bees (Hymenoptera: Apiformes) are by far the most important (Roubik 1995, Mader et al. 2011). Most pollinators do not collect nectar directly, but rather pollen attaches to their bodies incidentally when they visit a flower for nectar. On the other hand, only bees and masarine wasps (Hymenoptera: Vespidae) (Gess 1996) visit flowers for the purpose of collecting pollen for their offspring, and, in addition, exhibit flower constancy. Flower constancy is a behavior in which the foraging bee will tend to visit flowers of the same species in a given foraging event or over a longer period of time (Michener 2007). In addition to pollination enhancing behavior, bees have some anatomical adaptations which make them such effective pollinators. Probably the most important of these adaptations are the bee's plumose hairs. Plumose hairs carry an electrostatic charge (Michener 2007) that attracts pollen to them, meaning that most bees simply have to come close to the stamen of a plant to collect pollen from it. Additionally, many bees also have structures called scopal hairs located on the underside of the abdomen or the femur or tibia of the hind leg (Michener 1974). The scopal hairs may also form a corbicula, or pollen basket (as in the honey bees). In either case, these modified structures are used by the bees to concentrate pollen to a 
single location on their body for transportation (Michener 1974). Inevitably, in the process of visiting flowers, some amount of the collected pollen will make contact with the gynoecium of other flowers and pollination will occur.

\section{The Diversity of Bees and Their Origin}

According to the limited fossil record known for bees, bees arose sometime during the Cretaceous (146-76 million years ago). During this time, the first true flowering plants (angiosperms) arose. This new food source was an untapped niche that sphecid wasps quickly began to take over. How this happened is not entirely clear, but it is hypothesized that Psenulus, a sphecoid wasp genus which feeds on aphids (which produce a sugary secretion known as honeydew), was able to "hop" from sweet-tasting aphids to the sugary angiosperm nectar. Psenulus also shares a behavior with many ground nesting and cavity nesting bees, in that it lines its nest with a glandular secretion. Over a very long time sphecid wasps would develop new characteristics (scopal hairs, modified mouthparts) until they became entirely reliant on angiosperms as a food source, thus becoming the bees (O’Toole and Raw 1999).

Today, bees are one of the most diverse groups of animals on the planet, with an estimated 20,000 species globally. Following Michener (2007), bees form the superfamily Apoidea with the sphecoid wasps from which they arose, and are placed with that superfamily into the artificial taxon series Apiformes. The bees are frequently separated by the morphology of their mouthparts (i.e., short-tongued $[\mathrm{S}-\mathrm{T}]$ and long-tongued $[\mathrm{L}-\mathrm{T}]$ bees). According to Michener (2007) the S-T bees include the extant families Stenotritidae, Colletidae, Andrenidae, Halictidae, and Melittidae as well as the extinct families Dasypodaidae and Meganomiidae. The L-T bees include the families Megachilidae and Apidae, both of which are extant. In West Virginia there are 301 species (see Chapter 2) representing all extant families with the exception 
of Stenotritidae, which is restricted to Australia. There is only one species known from WV representing the family Melittidae, Melitta eickworti.

\section{Bee Sampling Methodologies}

While numerous methods may be utilized when the objective is to collect bees (transect walks, observation plots, malaise traps, etc.), utilization of pan traps is one of the most popular methods. Pan traps are small bowls or cups filled with a water and detergent. These bowls are painted a variety of colors, with the most common being white, yellow, or blue due to the attractiveness of these colors to bees (Kirk 1984, Leong and Thorp 1999, Toler et al. 2005), though other colors may be used in specific ecological scenarios (Stephen and Rao 2005). Paints often contain fluorescent pigment as they have been found more effective at catching bees than non-fluorescent paints (Bartholomew and Prowell 2005). The passive nature of pan traps and their good catch rate makes them ideal for studies where multiple days of collecting in an area are necessary. Pan traps do, however, have some downfalls. First, they have been shown to collect with a bias toward smaller bodied bees (Westphal et al. 2008, Wilson et al. 2008). Also, as with other passive sampling methods (Vega et al. 1990), non-target insects are frequently killed in pan traps which may be undesirable in some areas.

\section{Overview of Geostatistics}

When studying animal populations there are two components of particular interest to researchers: temporal patterns (how some variable changes over time) and spatial patterns (how some variable changes over space) (Liebhold et al. 2004). Geostatistics is a statistical procedure which can be used to describe the spatial dependence among sample values (Rossi et al. 1992). Geostatistics originated as a tool for predicting the location of minerals and ores based on sampled points. It was initially developed by Matheron (1963) based on ideas by Krige (1951) 
(see Haining et al. 2010). It is based on assumptions which are similar to those of traditional statistics: the assumption of normality and the assumption of stationarity. Where geostatistics differs from traditional statistics is in the assumption of independence. Traditional statistics assumes that all data are independent from each other; geostatistics assumes data are dependent on each other, and that data points which are closer together have a greater effect on each other than data points which are far from each other. In this way, geostatistics is much better suited to spatial analysis of ecological data when compared to traditional statistics. Ecological spatial data is most often found in gradients or patches and phenomena at one point may influence nearby points, or even points father away (Legendre and Fortin 1989).

Geostatistics models spatial dependence (autocorrelation) using variography, or other similar methods. It then uses the semivariogram to predict the values of samples at unknown locations and build a map. This process is called kriging (Rossi et al. 1992). A semivariogram can be used to determine the point at which samples become spatially independent (the range), estimate the sample variance (the sill), and determine microscale variation and experimental error (the nugget). The interpolation method by Krige (1951) utilizes the semivariogram model, and can be used not only to predict values at unsampled locations, but to easily visualize spatial distribution patterns. Analysis with kriging can create maps of the standard deviation of the points in the sampling domain, permitting the determination of confidence intervals (Cressie 1993). Because there is a known mean and standard deviation at each point on the map, variables at each point can be represented as random variables following a normal distribution (Zhang et al. 2009).

\section{General biology of Osmia cornifrons}


Osmia cornifrons (Hymenoptera: Megachilidae) is a Palearctic species occurring naturally in Japan, Korea, China, and eastern Russia. O. cornifrons is also found as an introduced species in the eastern United States, where it is managed for fruit orchard pollination.

Osmia cornifrons is a univoltine species (i.e., one generation per year). Adults become active in the early spring around the time apple (Malus domestica) trees begin to bloom. Male bees emerge first, and in managed populations many hundreds of males may be seen swarming outside of nest tubes as they await the opportunity to mate. Once females emerge copulation takes place for an hour or more (Batra 1978), and the female stores the males sperm in her spermatheca until she is prepared to nest. After mating has taken place males leave the area and spend the last few weeks of their lives in the field (Bosch and Kemp 2002, Matsumoto and Maejima 2010), where they may act as pollinators. Females leave the nesting area for a period of several days so that they may complete their ovarian development before beginning nest construction (Maeta 1978).

Once the female is prepared to nest she will seek out a nesting site, often returning to the area from which she emerged. In managed populations this will often be a cardboard straw or wooden block filled with holes, whereas naturally occurring $O$. cornifrons nest in broken reeds, bamboo, and similar structures. Once a nest is established, construction begins. The nest is a linear structure composed of a series of cells, with each cell consisting of a mass of pollen and nectar with an egg deposited into it. Cells are delineated by partitions constructed from mud gathered by the female bee. $O$. cornifrons nest construction has been described in detail by Maeta (1978) and McKinney and Park (2012).

Once eggs are laid, immature bees in the form of vermiform larvae will hatch within a short period of time and begin to consume the pollen/nectar mixture in their cells. Bees develop 
over five instars before entering a prepupal stage in early summer. $O$. cornifrons pupates shortly thereafter, and stays in the pupal stage throughout the summer before becoming an adult in the fall (Maeta 1978). Adult bees overwinter in their cocoons, as they require a chilling period to break their diapause (White et al. 2009) in following spring.

\section{Biology of Monodontomerus spp.}

Monodontomerus (Hymenoptera: Torymidae) wasps are parasitic wasps known to have hosts from three orders of insects: Diptera, Lepidoptera, and Hymenoptera (Grissel 2000), making these parasitoid wasps economically important both as biological control agents (Howard and Fiske 1911; McGugan and Coppel 1962; Washburn 1984; Digweed et al 2009; Van Driesche et al. 2010) and as pests (Eves 1970; Parker and Torchio 1980). Monodontomerus may be found in nearly all faunal regions of the world (Gahan 1941).

Monodontomerus spp. is a parasite of $O$. cornifrons. During spring emergence of $O$. cornifrons, Monodontomerus spp. detects host nests through detection of frass volatiles, especially acetic acid (Fillela et al. 2011). Once located, Monodontomerus enters the host nest and uses its ovipositor to probe through cocoons to find a suitable sized prepupal host. Monodontomerus can detect if another of its kind has already utilized a host, and will lay fewer eggs on these hosts. The exact mechanism through which this is accomplished is not fully understood. Monodontomerus larvae will hatch and consume the host, with a single host prepupa sustaining 3-51 larval wasps (Eves 1970). Once completed Monodontomerus will develop through multiple instars before pupating into an adult. Adults will emerge, often having 2-4 generations per year (Bosch and Kemp 2001), to locate a new host. The final generation of Monodontomerus each year will overwinter as a fully grown larvae in its host's nest before emerging the following spring (Bosch and Kemp 2001). 


\section{References Cited}

Abrol, D. P. 2012. Pollination biology: biodiversity conservation and agricultural production. Springer, New York, NY.

Bartholomew, C. S., and D. Prowell. 2005. Pan compared to malaise trapping for bees

(Hymenoptera: Apoidea) in a longleaf pine savanna. J. Kans. Entomol. Soc. 78: 390-392.

Batra, S. W. T. 1978. Osmia cornifrons and Pithitis smaragdula, two Asian bees introduced into the united states for crop pollination. Prov. Ivth Int. Symp. on Pollinat. Md. Agric. Exp. Sta. Spec. Misc. Publ. 1: 307-312.

Bosch, J., and W. P. Kemp. 2001. How to manage the blue orchard bee, Osmia lignaria, as an orchard pollinator. Washington, DC, Sustainable Agriculture Networks.

Bosch, J., and W. P. Kemp. 2002. Developing and establishing bee species as crop pollinators: the example of Osmia spp. (Hymenoptera: Megachilidae) and fruit trees. Bull. Entomol. Res. 92: 3-16.

Cressie, N. A. 1993. Statistics for spatial data. Wiley, New York, NY.

Digweed, S. C., C. J. K. MacQuarrie, D. W. Langor, D. J. M. Williams, J. R. Spence, K. L. Nystrom, and L. Morneau. 2009. Current status of invasive alien birch-leafmining sawflies (Hymenoptera: Tenthredinidae) in Canada, with keys to species. Can. Entomol. 141: 201-235.

Eves, J.D. 1970. Biology of Monodontomerus obscurus Westwood, a parasite of the alfalfa leafcutting bee, Megachile rotundata (Fabricius) (Hymenoptera: Torymidae; Megachilidae). Melandaria 4: 1-18. 
Fillela, I., J. Bosch, J. Llusià, R. Seco, and J. Peñuelas. 2011. The role of frass and cocoon volatiles in host location by Monodontomerus aeneus, a parasitoid of megachilid solitary bees. Environ. Entomol. 40(1): 126-131.

Free, J. B. 1993. Insect pollination of crops. Academic Press, San Diego, CA.

Gahan, A.B. 1941. A revision of the chalcid-flies of the genus Monodontomerus in the United States National Museum. Proc. of the U. S. Natl. Mus. 90: 461-482.

Gess, K. 1996. The pollen wasps: ecology and natural history of the Masarinae. Harvard University Press, Cambridge, MA.

Grissel, E. E. 2000. A revision of new world Monodontomerus Westwood (Hymenoptera: Chalcidoidea: Torymidae). Am. Entomol. Inst. 32(1): 1-90.

Haining, R. P., R. Kerry, and M. A. Oliver. 2010. Geography, spatial data analysis, and geostatistics: an overview. Geogr. Anal. 42: 7-31.

Howard, L. O., and W. F. Fiske. 1911. The importation into the United States of the parasites of the gypsy moth and the browntail moth. U. S. Dep. Agric. Bur. Entomol. Bull 91.

Kirk, W. D. J. 1984. Ecologically selective colored traps. Ecol. Entomol. 9: 35-41.

Klein, A. -M., B. E. Vassière, and J. H. Cane. 2007. Importance of pollinators in changing landscapes for world crops. Proc. of the Roy. Soc. B. 274(1608): 303-313.

Krige, R. 1951. A statistical approach to some basic mine valuation problems on the Witwaterstrand. J. Chem., Metallurgical, and Mining Soc. of South Afr. 52: 119-139.

Legendre, P., and M. -J. Fortin. 1989. Spatial pattern and ecological analysis. Vegetatio 80:107138.

Liebhold, A., W. D. Koenig, O. N. Bjørnstad. 2004. Spatial synchrony in population dynamics. Annu. Rev. Ecol. Evol. Syst. 35: 467-490. 
Leong, J. M., and R. W. Thorp. 1999. Colour-coded sampling: the pan trap color preferences of oligolectic and non-oligolectic bees associated with a vernal pool plant. Ecol. Entomol. 24: 329-335.

Mader, E., M. Shepherd, M. Vaughn, S. H. Black, and G. LeBuhn. 2011. Attracting native pollinators: protecting North America's bees and butterflies. Storey Publishing, North Adams, MA.

Maeta, Y. 1978. Comparative studies on the biology of the genus Osmia of Japan, with special reference to their managements for pollinations of crops (Hymenoptera: Megachilidae). Bull. Tohoku Natl. Agric. Exp. Stn. No. 57. 1-242.

Matheron, G. 1963. Principles of geostatistics. Econ. Geol. 58:1246-1266.

Matsumoto, S., and T. Maejima. 2010. Several new aspects of the foraging behavior of Osmia cornifrons in an apple orchard. Psyche 2010: 1-6.

McGugan, B. M., H. C. Coppel.1962. Biological control of forest insects - 1910-1958. In: A Review of the Biological Control Attempts Against Insects and Weeds in Canada. Commonwealth Agricultural Bureaux Franham Royal, UK, pp. 35-127.

McKinney, M. I., and Y. -L. Park. 2012. Nesting activity and behavior of Osmia cornifrons (Hymenoptera: Megachilidae) elucidated using videography. Psyche 2012: 1-8.

Michener, C.D. 1974. The social behavior of the bees: a comparative study. The Belknap Press of Harvard University Press. Cambridge, MA.

Michener, C. D. 2007. The bees of the world, $2^{\text {nd }}$ ed. The Johns Hopkins University Press, Baltimore, MD.

Morse, R. A. and N. W. Calderone. 2000. The value of honey bees as pollinators of U.S. crops in 2000. Bee Culture. 128: 2-15. 
Nabhan, G. P., and S. Buchmann. 1997. Nature's services: Societal dependence on natural ecosystems. Island Press, Washington, DC.

O’Toole, C., and A. Raw. 1999. The bees of the world. Facts on File, Inc., New York, NY.

Parker, F. D., and P. F. Torchio. 1980. Management of wild bees, pp. 144-160. Beekeeping in the United States. United States Department of Agriculture. Handbook, 335: 1-193.

Rossi, R. E., D. J. Mulla, A. G. Journel, and E. H. Franz. 1992. Geostatistical tools for modeling and interpreting ecological spatial dependence. Ecol. Monogr. 62: 277-314.

Roubik, D. W. 1995. Pollination of cultivated plants in the tropics. Food and Agricultural Organization of the United Nations, Rome, Italy.

Stephen, W. P. and S. Rao. 2005. Unscented color traps for non-Apis bees (Hymenoptera: Apiformes). J. Kans. Entomol. Soc. 78: 373-380.

Toler, T. R., E. W. Evans, and V. J. Tepedino. 2005. Pan trapping for bees (Hymenoptera: Apiformes) in Utah's west desert: the importance of color diversity. Pan-Pac. Entomol. 81: 103-113.

Van Driesche, R. G., R. I. Carruthers, T. Center, M. S. Hoddle, J. Hough-Goldstein, L. Morin, L. Smith, D. L. Wagner, B. Blossey, V. Brancatini, R. Casagrande, C. E. Causton, J. A. Coetzee, J. Cuda, J. Ding, S. V. Fowler, J. H. Frank, R. Fuester, J. Goolsby, M. Grodowitz, T. A. Heard, M. P. Hill, J. H. Hoffmann, J. Huber, M. Julien, M. T. K. Kairo, M. Kenis, P. Mason, J. Medal, R. Messing, R. Miller, A. Moore, P. Neuenschwander, R. Newman, H. Norambuena, W. A. Palmer, R. Pemberton, A. Perez Panduro, P. D. Pratt, M. Rayamajhi, S. Salom, D. Sands, S. Schooler, M. Schwarzländer, A. Sheppard, R. Shaw, P. W. Tipping, R. D. van Klinken. 2010. Classical biological control for the protection of agricultural ecosystems. Biol. Contr. 54: S2-S33. 
Vega, F. E., P. Barbosa, and A. P. Panduro. 1990. An adjustable water-pan trap for simultaneous sampling of insects at different heights. Fla. Entomol. 73: 656-660.

Washburn, J.O. 1984. The gypsy moth and its parasites in North America: A community in equilibrium? Am. Nat. 124(2): 288-292.

Westphal, C., R. Bommarco, G. Carré, E. Lamborn, N. Morrison, T. Petanidou, S. G. Potts, S. P. M. Roberts, H. Szentgyörgyi, T. Tscheulin, B. E. Vaissière, M. Woyciechowski, J. C. Niesmeijer, W. E. Kunin, J. Settele, and I. Steffan-Dewenter. 2008. Measuring bee diversity in different European habitats and biogeographical regions. Ecol. Monogr. 78: 653-671.

Wilson, J. S., T. Griswold, and O. J. Messinger. 2008. Sampling bee communities (Hymenoptera: Apiformes) in a desert landscape: are pan traps sufficient. J. Kans. Entomol. Soc. 81: 288-300.

White, J. B., Y. -L. Park, and Y.-S. Son. 2009. Temperature-dependent emergence of Osmia cornifrons (Hymenoptera: Megachilidae) adults. J. Econ. Entomol. 102: 2026-2032.

Zhang, J., J. Zhang, and N. Yao. 2009. Geostatistics for spatial uncertainty characterization. Geospatial Inf. Sci. 12: 7-12. 


\title{
CHAPTER 2: CHECKLIST OF THE BEES OF WEST VIRGINIA
}

\begin{abstract}
To advance the study of West Virginia's native pollinators, we here present the first annotated, distributional checklist of the state's 301 recorded bee species (in six families and 41 genera). For each of these species, we provide distributional data at the county level, while also presenting known host plant associations, notes on rare or interesting records, and brief discussions of biogeographic patterns and status. We also briefly review the history of bee research in the state, discuss gaps in our current knowledge, and explore avenues for future research. Checklist data were compiled from literature records, databased specimens in natural history collections (most of which are identified and presented here for the first time), and recent field collections by the authors.
\end{abstract}

\section{Introduction}

The irregular borders of the state of West Virginia-ranging from approximately $37.20^{\circ}$ to $40.64^{\circ}$ latitude, and $-77.73^{\circ}$ to $-82.64^{\circ}$ longitude - enclose a geographic mosaic of northern, southern, and boreal woodlands shared by elements of both northern and southern regional faunas (Strausbaugh and Core 1978, Wheeler et al. 1983). Located in the heart of the Appalachian Mountains and associated foothills (a.k.a., the "Southern Appalachian Highlands" [Blauch 1975]), the state's dramatic topography presents a wide range of microclimates, including fertile alluvial valleys (e.g., the Mid-Ohio, the Kanawha), high elevation valleys (e.g., Canaan), frost pockets (e.g., Cranesville Swamp), mixed mesophytic forests (sensu Braun 1950; located throughout the central and southern parts of the state); high elevation bogs (e.g., Cranberry Glades), and boreal forest relicts (e.g., Spruce Knob and Dolly Sods). Resource extraction and anthropogenic disturbance in West Virginia — beginning with extensive 
nineteenth- and early-twentieth-century clear-cut logging (Pauley 2008), and culminating in present day mountaintop-removal coal mining practices (Palmer et al. 2010) - have altered these natural features. However, many pristine sites remain in protected areas throughout the state, such as Gaudineer Knob, Cathedral State Park, Carnifex Ferry State Park, and Koontz Bed. Habitats in West Virginia with high native biodiversity (Wheeler et al. 1983) are exemplified by Appalachian cove forests, which are noted for their vascular plant fauna, among the richest of any temperate ecoregion on the planet (Strausbaugh and Core 1977). The state's extensive forests, aided by low population densities and limited economic development, remain a stronghold for many poorly known and/or declining species such as the Cheat Mountain Salamander, Plethodon nettingi. Among ornithologists and birders, for example, the mountains of West Virginia are well known for their diversity of "northern" wood-warblers (subfamily: Parulinae), and the state also has the northernmost breeders of the "southern" rhododendronassociated Swainson's Warbler (Limnothyplis swainsonii) (Brooks and Legg 1942) and significant populations of the declining Cerulean Warbler (Setophaga cerulea; Weakland and Wood 2005).

Relatively few studies have investigated the diversity of West Virginia's insect fauna, and no previous reports on West Virginia's bee fauna have been written. Insect groups which have been reported on include Trichoptera (Glover and Tarter 1990, Tarter 1990), Ephemeroptera (Tarter and Kirchner 1975, Fisher and Tarter 1988), Plecoptera (Tarter et al. 2006, Tarter et al. 2010), Rhopalocera (Drees and Butler 1978), Miridae (Wheeler et al. 1983), Culicidae (Amrine and Butler 1978, Joy et al. 1994), Tabanidae (Drees et al. 1980), Corydalidae (Tarter et al. 2013), and Sesiidae (Albu 1997). While the importance of pollinators for ecosystem health has never been in question (Klein et al. 2007, Hoshiba and Sasaki 2008, Mader et al. 
2011), recent concerns about the health of managed honey bee (Apis mellifera L.) populations (Stokstad 2007), declines in native pollinators (Kearns et al. 1998), and the impact of changing climate on interactions between bees and their host plants have brought renewed attention to this important aspect of environmental health. However, the conclusions from these studies are not yet clear, as the causes of bee declines are complex and often taxon specific (The White House 2014), and most eastern North American species have been shown to persist until recent times (Colla et al. 2012, Bartomeus et al. 2013).

State checklists are a first step toward establishing baseline data on species distributions and abundance, but relatively few detailed distributional checklists have been published for U.S. states, including Colorado (Scott et al. 2011), Pennsylvania (Donovall and van Engelsdorp 2010), and Wisconsin (Wolf and Ascher 2008). Mitchell $(1960,1962)$ summarized state occurrences for all eastern U.S. states and provided quite comprehensive data for his home state of North Carolina, but records for many other states, including West Virginia, were fragmentary, and few were available for citation in subsequent catalogs (Hurd 1979, Moure and Hurd 1987). State records and individual specimen records are now available for all states online (www.discoverlife.org; including many records from the mid-Atlantic region compiled by Sam Droege and colleagues), but need to be further validated and documented and placed in context. These efforts have been facilitated by development of large-scale collaborative specimen databasing projects spearheaded by the American Museum of Natural History and collaborating institutions (Schuh et al. 2010, Arthropod Easy Capture 2013).

Detailed, print checklists for additional states facilitate evaluation of the conservation status of native bees as a foundation for practical conservation measures. In the present study, we 
compile specimen and literature records, informed by our own surveys within this and neighboring states, to compile the first distributional checklist of the bees of West Virginia.

\section{Materials and Methods}

The checklist presented here was compiled from multiple sources including 1) records from the databases of the American Museum of Natural History and collaborating institutions (see Table 1) and the United States Geological Survey, including recent (i.e., since 2012) records by Sam Droege and Jane Whitaker spanning many of the eastern counties, and 2) sweep netting and pan trapping work in Canaan Valley National Wildlife Refuge by Matthew McKinney. Specimens from BugGuide (www.bugguide.net, accessed 2013) were also added if identifications could be confirmed from the images. Records spanned 99 years, with the earliest record from 1914 (a specimen of Augochlorella aurata from Greenbrier Co.). In total, 17,462 records were acquired from the following databases: American Museum of Natural History bee database $(n=1415)$, the USGS bee monitoring program database $(n=15,205)$, the BBSL database $(n=529)$, the Natural Museum of Natural History $(n=203)$, and the Ohio State University Collection $(\mathrm{n}=110)$. Additional species occurrence information was obtained through taxonomic revisions. Questionable records were omitted unless a voucher specimen was obtained (Table 2).

The hierarchical classification of family-group names - i.e., families, subfamilies, and — follows Engel (2005), with minor modifications as detailed in Scott et al. (2011). Generic and subgeneric classification generally follows Michener (2007), with some exceptions as detailed in Scott et al. (2011) and subsequent revisionary studies (e.g., Gibbs et al. 2013, Sedivy et al. 2013). List nomenclature follows Ascher and Pickering (2016), with junior synonyms excluded.

To create a map depicting the various collections made throughout the state, the number of unique collection events within each county was determined from all available data, and 
converted into a percentage of the total number of unique collecting events. These data were appended to the county layer in ArcGIS 10.1 and displayed in categories of three percent increments. Map layers were obtained from the WV State GIS Data Clearinghouse (http://wvgis.wvu.edu/data/data.php). To identify historical localities used in the mapping process, both Google Earth (http://www.google.com/earth/) and the United States Board on Geographic Names (USGS/USDI BGN) (http://geonames.usgs.gov/domestic/index.html) were used.

\section{Results}

Families are listed alphabetically, and are separated at the subfamily and tribe levels.

Next to each family, subfamily, and tribe is a number indicating the number of species present at that level of the taxonomic hierarchy. Species are grouped by genus and subgenus within each tribe and listed alphabetically. Subspecies, when applicable, are listed beneath the species entry. Each species listed may be followed by superscript code indicating important information about that species (see the key preceding the list). For each species all county records are listed, with each county record being followed by a museum collection code (Table 1). Museum collection codes indicate the source(s) of each county record, and may be useful to researchers doing related work in the future. At the end of each species entry there may be information related to the aforementioned superscript codes or other notes. In either case, each of these additional entries begins with a bold heading followed by a semi-colon.

The map depicting collection effort across West Virginia (Figure 1), as reflected in our combined dataset, shows that there has been heavy collecting in the eastern portion of the state, and that many collections have been made in Monongalia and Kanawha counties relative to the other counties in the state. These results are not surprising given that Monongalia Co. is home to largest research institution in the state, West Virginia University, and that Kanawha Co. holds 
the state capitol of Charleston. What is somewhat surprising is the lack of any collecting, historical or recent, in the central counties of Lewis, Braxton, and Webster.

Key:

1-Single specimen

2 - Two specimens

I - Single collection event (> 1 specimen)

E - Exotic

N - Northern species

$\mathbf{S}$ - Southern species

o _ Oligolectic

P - Parasitic

\section{FAMILY ANDRENIDAE [71]}

\section{Subfamily: Andreninae [64]}

Tribe: Andrenini [64]

Andrena (Andrena) carolina Viereck, $1909^{\circ}$ - Hardy [USGS], Tucker [WVU]. Host: Vaccinium (Ericaceae).

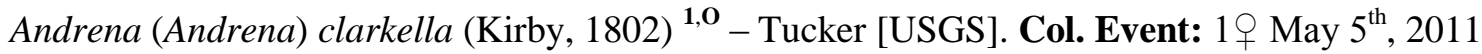
@ 39.1141, -79.4894; Davis at Blackwater Falls State Park; J. Whitaker. Host: Vaccinium (Ericaceae).

Andrena (Andrena) mandibularis Robertson, $1892^{2}$ - Randolph [USGS], Wetzel [RUAC]. Andrena (Andrena) milwaukeensis Graenicher, 1903 - Greenbrier [USGS], Hardy [USGS], Pocahontas [BBSL; USGS], Randolph [USGS], Tucker [USGS].

Andrena (Andrena) rufosignata Cockerell, 1902 - Pendleton [USGS], Randolph [USGS], Tucker [USGS].

Andrena (Andrena) thaspii Graenicher, 1903 - Hardy [USGS], Pendleton [BBSL], Pocahontas [WVU], Tucker [USGS]. Notes: Mitchell's (1960) record of Andrena (Conandrena) durangoensis Viereck and Cockerell 1914, was likely based on a misidentified male of $A$. thaspii. 
Andrena (Andrena) tridens Robertson, 1902 - Hardy [USGS], Jefferson [CUIC], Tucker [USGS].

Note: Also listed by Mitchell (1960) as A. (Gymnandrena) bisignata.

Andrena (Archiandrena) banksi Malloch, $1917^{\mathbf{1}}$ - Hardy [USGS]. Col. Event: $1 \bigcirc^{\lambda}$ June, $2007 @$ 38.9166, -78.8166; 3 mi. NE of Mathias; D. Smith.

Andrena (Callandrena s.1.) aliciae Robertson, $1891^{\circ}$ - Greenbrier [USGS], Hampshire [USGS], Jefferson [USGS]. Host: Asteraceae.

Andrena (Callandrena s.1.) asteris Robertson, $1891^{\circ}$ - Cabell [USGS], Hampshire [USGS],

Kanawha [NMNH; NMNH; WVU], Monongalia [WVU], Pleasants [USGS]. Hosts:

Asteraceae

Andrena (Callandrena s.1.) gardineri Cockerell, $1906^{\circ}$ - Hampshire [USGS]. Col. Event: 5 +

May 27 th $^{\text {}} 2006$ - May 28, 2006 @ 39.334, -78.4559; Buffalo Community Camp. Hosts:

Asteraceae.

Andrena (Callandrena s.1.) krigiana Robertson, $1901^{2}$ - Hampshire [BBSL; USGS], Hardy

[USGS].

Andrena (Callandrena s.1.) placata Mitchell, $1960^{\text {2,I }}$ - Pleasants [USGS]. Col. Event: 2 Sept.

$15^{\text {th }}, 2011 @ 39.4063,-81.204$; Middle Island near St. Marys; J. Whitaker.

Andrena (Callandrena s.1.) simplex Smith, $1853^{\circ}$ - Greenbrier [USGS], Hampshire [USGS],

Randolph [USGS], Tucker [USGS]. Host: Asteraceae.

Andrena (Cnemidandrena) canadensis Dalla Torre, $1896^{\text {2,N }}$ - Pendleton [USGS], Tucker

[USGS].

Andrena (Cnemidandrena) chromotricha Cockerell, $1899^{\mathbf{1}}$ - Pocahontas [USGS]. Col. Event:

$19 @ 38.3909,-79.9217$; Cass, Greenbrier Trail; J. Whitaker.

Andrena (Cnemidandrena) hirticincta Provancher, $1888^{\circ}$ - Barbour [USGS], Doddridge

[USGS], Grant [USGS], Hampshire [USGS], Monongalia [WVU], Pleasants [USGS],

Preston [WVU], Tucker [USGS]. Host: Asteraceae. 
Andrena (Cnemidandrena) nubecula Smith, $1853^{\circ}$ - Pendleton [USGS], Preston [WVU], Upshur [BugGuide]. Host: Asteraceae.

Andrena (Derandrena) ziziaeformis Cockerell, 1908 - Hardy [USGS], McDowell [USGS],

Preston [USGS], Tucker [USGS].

Andrena (Euandrena) geranii Robertson, 1891 - Pendleton [USGS], Pocahontas [USGS],

Randolph [USGS].

Andrena (Euandrena) phaceliae Mitchell, $1960^{1}$ - Hampshire [USGS]. Col. Event: 1 ㅇ May $27^{\text {th }}, 2005$ @ 39.2681, -78.4484; Capon Bridge; S. Droege.

Andrena (Euandrena) polemonii Robertson, $1891^{1}$ - Lincoln [USGS]. Col. Event: 1 ㅇ April $12^{\text {th }}$, 2008 @ 38.2893333, -82.196; West Hamlin; J. Whitaker.

Andrena (Gonandrena) fragilis Smith, 1853 - Barbour [USGS], Monroe [WVU], Preston [USGS], Randolph [USGS].

Andrena (Holandrena) cressonii Robertson, 1891 - See subspecies.

cressonii Robertson, 1891 - Berkeley [USGS], Hampshire [USGS], Hardy [USGS], Jefferson [BBSL; USGS], Morgan [USGS; UNHP], Ohio [USGS], Pendleton [USGS], Pleasants [USGS], Raleigh [CUIC], Ritchie [USGS], Wetzel [RUAC], Wood [USGS]. Andrena (Iomelissa) violae Robertson, $1891^{\circ}$ - Berkeley [USGS], Cabell [USGS], Hampshire [USGS], Jefferson [BBSL; USGS], Kanawha [WVU], Lincoln [USGS], Monongalia [WVU], Morgan [UNHP], Pendleton [USGS], Pleasants [USGS], Randolph [USGS], Ritchie [USGS], Tucker [USGS], Wood [USGS]. Host: Viola (Violaceae). Andrena (Larandrena) miserabilis Cresson, 1872 - Berkeley [USGS], Grant [USGS], Hampshire [USGS], Jefferson [BBSL], Lincoln [USGS], Nicholas [BBSL], Ohio [BBSL; USGS], Pendleton [USGS], Pleasants [USGS], Pocahontas [USGS], Preston [USGS], Randolph [USGS], Tucker [USGS; WVU], Wayne [USGS], Wetzel [RUAC], Wood [USGS]. Andrena (Leucandrena) erythronii Robertson, $1891^{\circ}$ - Randolph [USGS], Tucker [WVU], Wetzel [RUAC], Wood [USGS]. Host: Erythronium (Liliaceae). 
Andrena (Melandrena) barbara Bouseman and LaBerge, 1979 - Berkeley [USGS], Hampshire [USGS], Jefferson [USGS].

Andrena (Melandrena) carlini Cockerell, 1901 - Berkeley [USGS], Greenbrier [USGS],

Hampshire [USGS], Hardy [USGS], Jefferson [BBSL; USGS], Kanawha [BBSL], Monongalia [WVU], Morgan [USGS], Ohio [USGS], Pendleton [BBSL; USGS], Pleasants [USGS], Randolph [USGS], Tucker [USGS; WVU], Wetzel [RUAC]. Andrena (Melandrena) commoda Smith, 1879 - Grant [USGS], Hardy [USGS], Randolph [USGS].

Andrena (Melandrena) confederata Viereck, $1917^{\mathbf{1}}$ - Summers [BBSL]. Col. Event: 1 q May $22^{\text {nd }}, 1976 @ 37.544342,-80.959957$; Pipestem; collector unknown.

Andrena (Melandrena) illini Bouseman and LaBerge, $1979^{\text {S }}$ - Pleasants [USGS], Randolph [USGS].

Andrena (Melandrena) nivalis Smith, $1853^{\mathbf{N}}$ - Greenbrier [USGS], Hardy [BBSL; USGS],

Nicholas [BBSL], Pocahontas [BBSL; USGS], Preston [USGS], Randolph [USGS;

YALE], Tucker [USGS; WVU].

Andrena (Melandrena) pruni Robertson, 1891 - Berkeley [BBSL], Fayette [BBSL], Greenbrier [USGS], Hampshire [USGS], Hardy [BBSL; USGS], Jefferson [BBSL; USGS], McDowell [USGS], Monroe [AMNH], Nicholas [BBSL], Ohio [USGS], Pendleton [BBSL; USGS], Randolph [USGS], Summers [BBSL], Taylor [BBSL]. Andrena (Melandrena) vicina Smith, 1853 - Greenbrier [USGS], Hampshire [USGS], Hardy [USGS], Jefferson [USGS], Morgan [USGS], Pendleton [USGS], Pleasants [USGS], Wetzel [RUAC].

Andrena (Micrandrena) personata Robertson, 1879 - Hampshire [USGS], Morgan [BBSL], Pendleton [BBSL], Randolph [USGS], Tucker [USGS].

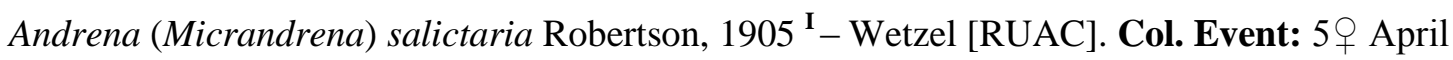
$22^{\text {nd }}, 1979$ @ 39.683,-80.629; 9 mi. W of Hundred; R. B. Roberts. 
Andrena (Micrandrena) ziziae Robertson, 1891 - Hardy [USGS], McDowell [USGS], Randolph [USGS].

Andrena (Plastandrena) crataegi Robertson, 1893 - Berkeley [USGS], Gilmer [AMNH],

Greenbrier [USGS; AMNH], Hardy [USGS], Jefferson [USGS], Monongalia [WVU], Morgan [USGS], Pendleton [USGS], Pocahontas [USGS], Preston [USGS; WVU], Randolph [USGS], Tucker [USGS; WVU].

Andrena (Ptilandrena) erigeniae Robertson, $1891^{\circ}$ - Berkeley [USGS], Grant [USGS],

Hampshire [USGS], Hardy [USGS], Jefferson [BBSL; USGS], Kanawha [BugGuide], McDowell [USGS], Morgan [USGS], Pendleton [BBSL; USGS], Pleasants [USGS], Randolph [USGS], Tucker [USGS], Wetzel [RUAC], Wood [USGS]. Host: Claytonia (Moniaceae).

Andrena (Rhacandrena) brevipalpis Cockerell, 1930 - Hampshire [USGS], Hardy [UCD], Jackson [AMNH], Kanawha [NMNH], Mineral [NMNH], Monongalia [WVU], Pocahontas [BBSL], Preston [USGS].

Andrena (Rhacandrena) robertsonii Dalla Torre, 1896 - Mineral [UCD], Preston [USGS],

Wetzel [RUAC].

Andrena (Scaphandrena) arabis Robertson, $1897^{2}$ - Hampshire [USGS], Wood [USGS]. Andrena (Scrapteropsis) alleghaniensis Vierick, $1907^{\mathbf{1}}$ - Hampshire [USGS]. Col. Event: 1 ㅇ May 26 ${ }^{\text {th }}, 2007$ @ 39.2321, -78.4642; Timber Ridge Camp; S. Droege. Andrena (Scrapteropsis) atlantica Mitchell, $1960{ }^{1}$ - Hampshire [USGS]. Col. Event: 19 May $29^{\text {th }}, 2005 @ 39.3353,-78.4557$; Buffalo Gap; S. Droege.

Andrena (Scrapteropsis) imitatrix Cresson, 1872 - Barbour [BBSL], Hampshire [USGS], Hardy [USGS], Jefferson [USGS], Kanawha [BBSL], Lincoln [USGS], Pendleton [USGS], Pleasants [USGS], Pocahontas [USGS], Randolph [USGS], Tucker [WVU], Wayne [USGS], Wetzel [RUAC], Wood [USGS]. 
Andrena (Scrapteropsis) morrisonella Vierick, 1917 - Hardy [USGS], Jefferson [USGS],

Pendleton [USGS], Tucker [USGS].

Andrena (Simandrena) nasonii Robertson, 1895 - Berkeley [BBSL; USGS], Grant [USGS],

Greenbrier [USGS], Hampshire [USGS], Hardy [USGS], Jefferson [CAES; CUIC;

USGS], Lincoln [USGS], McDowell [USGS], Monongalia [WVU], Pendleton [BBSL;

USGS], Pleasants [USGS], Pocahontas [USGS], Preston [USGS], Randolph [USGS],

Ritchie [USGS], Tucker [USGS; WVU], Wetzel [RUAC], Wood [USGS].

Andrena (Simandrena) wheeleri Graenicher, $1904^{\mathbf{1 , N}}$ - Tucker [WVU]. Col. Event: 1 q May

$17^{\text {th }}, 2012$ @ 39.13286, -79.44238; Canaan Valley National Wildlife Refuge, Camp 70

Rd., 1.2 mi. E of Hwy 32; M. McKinney and S. Baek.

Andrena (Taeniandrena) wilkella (Kirby, 1802) - Gilmer [AMNH], Greenbrier [AMNH; BBSL],

Hampshire [USGS], Hardy [USGS], Jackson [AMNH; BBSL], Jefferson [CAES; WVU],

Kanawha [AMNH], Lincoln [AMNH], McDowell [USGS], Monongalia [WVU], Morgan

[USGS], Pendleton [USGS], Pocahontas [USGS], Preston [USGS], Randolph

[BugGuide; USGS], Roane [AMNH], Tucker [USGS; WVU], Tyler [WVU].

Andrena (Thysandrena) bisalicis Vierick, 1908 - Jefferson [BBSL], Pendleton [USGS], Tucker

[USGS; WVU], Wetzel [RUAC].

Andrena (Thysandrena) w-scripta Vierick, $1904^{\mathbf{N}}$ - Tucker [WVU], Pendleton [USGS]. Note:

Pendleton Co. record unconfirmed.

Andrena (Trachandrena) ceanothi Vierick, $1917^{\mathbf{1}}-$ Hardy [USGS]. Col. Event: 19 June $9^{\text {th }}$, $2006 @ 38.916667,-78.816667 ; 3$ mi. NE of Mathias; D. Smith.

Andrena (Trachandrena) forbesii Robertson, 1891 - Greenbrier [USGS], Hampshire [USGS], Jefferson [USGS], Morgan [USGS], Pendleton [USGS], Randolph [USGS], Tucker [USGS; WVU], Wetzel [RUAC].

Andrena (Trachandrena) heraclei Robertson, 1897 - Hampshire [USGS], Hardy [USGS], Jackson [AMNH], Morgan [BBSL; USGS], Pendleton [BBSL], Preston [BBSL]. 
Andrena (Trachandrena) hippotes Robertson, 1895 - Jefferson [USGS], Ohio [USGS], Randolph [USGS], Tucker [USGS; WVU], Wetzel [RUAC].

Andrena (Trachandrena) mariae Robetson, $1891^{\mathbf{1}}-$ Hardy [BBSL]. Col. Event: 19 May $7^{\text {th }}$, $1966 @ 39.069969,-78.967899 ;$ Moorefield; collector unknown.

Andrena (Trachandrena) nuda Robertson, 1891 - Greenbrier [USGS], Hampshire [USGS],

Hardy [USGS], Jackson [AMNH], Morgan [USGS], Nicholas [WVU], Ohio [USGS], Preston [USGS], Wetzel [RUAC].

Andrena (Trachandrena) rugosa Robertson, 1891 - Berkeley [USGS], Grant [USGS], Greenbrier [USGS], Hardy [USGS], Logan [USGS], Pendleton [USGS], Pocahontas [USGS], Tucker [USGS; WVU], Wetzel [RUAC].

Andrena (Trachandrena) sigmundi Cockerell, $1902{ }^{1}$ - Greenbrier [USGS]. Col. Event: 1 q May 30 ${ }^{\text {th }}, 2010$ @ 38.0875, -80.4518; Greenbrier Rd. on Babar Mt. near Trout; J. Whitaker. Note: Unconfirmed.

Andrena (Trachandrena) spiraeana Robertson, 1895 - Berkeley [USGS], Calhoun [AMNH], Greenbrier [USGS], Hampshire [USGS], Hardy [USGS], Preston [USGS]. Andrena (Trachandrena) virginiana Mitchell, 1960 - Tucker [WVU]. Andrena (Tylandrena) erythrogaster (Ashmead, 1890) ${ }^{\mathbf{1}, \mathbf{o}}$ - Monongalia [WVU]. Col. Event: 1 q date unknown@ 39.62944,-79.95583; Morgantown; collector unkown. Host: Salix (Salicaceae).

Andrena (Tylandrena) perplexa Smith, 1853 - Berkeley [USGS], Grant [USGS], Hampshire [USGS], Jefferson [CAES; USGS], Kanawha [BBSL], Nicholas [BBSL], Ohio [USGS], Pendleton [USGS], Pocahontas [USGS], Randolph [USGS], Wetzel [RUAC].

\section{Subfamily: Panurginae [7]}

Tribe: Calliopsini [1]

Calliopsis (Calliopsis) andreniformis Smith, 1853 - Hampshire [USGS], Hardy [NMNH; USGS], Jefferson [USGS], Mercer [BugGuide], Pleasants [USGS], Preston [USGS], Raleigh [BBSL], 
Randolph [USGS], Ritchie [USGS], Roane [AMNH], Tucker [USGS; WVU], Wayne [BBSL].

Tribe: Panurgini [1]

Subtribe: Perditina [1]

Perdita (Perdita) octomaculata (Say, 1824) $)^{2, \mathrm{I}, \mathrm{O}}-$ See subspecies.

octomaculata (Say, 1824) - Preston [AMNH]. Col. Event: $2 ð$ July 29 $9^{\text {th }}, 1977 @$

39.30984, -79.6453; 18 mi. E of Grafton (Taylor Co.), Cheat River; N. L. Herman. Host:

Asteraceae.

Tribe: Protandrenini [5]

Pseudopanurgus andrenoides (Smith, 1853) ${ }^{\mathbf{1 , 0}}-$ Monongalia [WVU]. Col. Event: 1 \% Sept. 30 ${ }^{\text {th }}$, 1976 @ locality unknown; M. Stewart. Host: Asteraceae.

Pseudopanurgus compositarum (Robertson, 1893) ${ }^{\mathbf{2}, \mathbf{0}}$ - Cabell [BBSL; USGS], Clay [BBSL],

Pleasants [USGS]. Host: Asteraceae.

Pseudopanurgus labrosiformis (Robertson, 1998) - Doddridge [USGS], Hampshire [USGS].

Pseudopanurgus labrosus (Robertson, 1895) ${ }^{1}$ - Hampshire [USGS]. Col. Event: $1 \overbrace{}^{\widehat{A}}$ Aug. $15^{\text {th }}$, $2004 @ 39.322,-78.4302 ;$ Buffalo Gap; S. Droege.

Pseudopanurgus virginicus (Cockerell, 1907) ${ }^{2, \mathrm{I}, \mathrm{S}}-$ Hardy [NMNH; USGS]. Col. Event: $2 q$ June $7^{\text {th }}, 2007$ @ 38.9166, -78.8166; 3 mi. NE of Mathias; D. Smith.

\section{FAMILY APIDAE [71]}

\section{Subfamily: Apinae [36]}

Tribe: Anthophorini [5]

Anthophora (Clisodon) terminalis Cresson, 1869 - Hampshire [USGS], Hardy [USGS], Mason

[WVU], McDowell [USGS], Monongalia [WVU], Pendleton [USGS], Pocahontas

[BBSL], Randolph [USGS].

Anthophora (Lophanthophora) ursina Cresson, $1869^{2}$ - Hampshire [USGS]. 
Anthophora (Melea) abrupta Say, 1838 - Berkeley [BBSL], Fayette [WVU], Hardy [BBSL; NMNH; USGS], Mason [BBSL], McDowell [USGS], Mineral [BBSL], Pocahontas [USGS], Randolph [USGS].

Anthophora (Melea) bomboides Kirby, 1838 - Hampshire [USGS], Hardy [USGS], Monroe [WVU].

Habropoda laboriosa (Fabricius, 1804) ${ }^{\mathbf{1}}-$ Jefferson [CUIC]. Col. Event: $1 \widehat{\sigma}^{A}$ April $2^{\text {nd }}, 1921 @$ 39.29388, -77.78583; Millville; J. C. Bradley.

Tribe: Apini [1]

Apis (Apis) mellifera (Linnaeus, 1758) ${ }^{\mathbf{E}}$ - Barbour [USGS], Berkeley [USGS], Cabell [USGS], Clay [WVU], Doddridge [USGS], Grant [USGS], Greenbrier [USGS], Hampshire [USGS], Hardy [USGS; WVU], Jefferson [USGS], Lincoln [USGS], McDowell [USGS], Monongalia [WVU], Monroe [WVU], Ohio [USGS], Pendleton, [USGS], Pleasants [USGS], Pocahontas [USGS], Preston [WVU], Raleigh [USGS], Randolph [USGS], Ritchie [USGS], Tucker [USGS; WVU], Wayne [USGS], Wood [WVU], Wyoming [USGS]. Note: Expected to occur in all counties.

Tribe: Bombini [15]

Bombus (Bombias) auricomus (Robertson, 1903) - Cabell [BBSL], Hardy [BBSL], Jefferson [USGS], Lincoln [BBSL; USGS], Monongalia [NMNH; WVU], Randolph [WVU], Roane $[\mathrm{AMMH}]$.

Bombus (Bombus) affinis Cresson, 1863 - Fayette [BBSL], Hardy [BBSL; UCD], Jefferson [BBSL], Kanawha [BBSL], Monongalia [WVU], Nicholas [BBSL], Pendleton [WVU], Pocahontas [BBSL; OSUC; WVU], Preston [BBSL], Randolph [BBSL; OSUC; UCD], Roane [BBSL], Wayne [UCD].

Bombus (Bombus) terricola Kirby, $1837^{\mathbf{N}}$ - Pendleton [OSUC; BBSL], Pocahontas [BBSL]. 
Bombus (Cullumanobombus) griseocollis (DeGeer, 1773) - Berkeley [USGS], Calhoun [AMNH; USGS], Doddridge [USGS], Gilmer [AMNH], Greenbrier [USGS], Hampshire [USGS], Hardy [USGS; WVU], Harrison [USGS], Jackson [AMNH], Jefferson [USGS], Kanawha [AMNH], Lincoln [WVU], Monongalia [WVU], Ohio [USGS], Pendleton [USGS;

WVU], Pleasants [USGS], Pocahontas [OSUC], Preston [WVU], Randolph [USGS], Ritchie [USGS], Roane [AMNH], Tucker [USGS], Tyler [USGS].

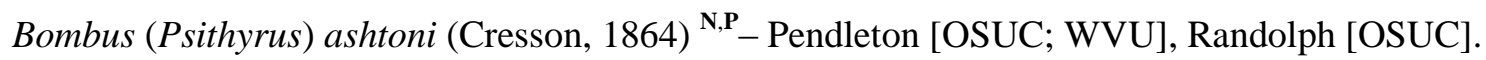
Bombus (Psithyrus) citrinus (Smith, 1853) ${ }^{\mathbf{P}}$ - Hampshire [USGS], Hardy [USGS], Morgan [WVU], Pendleton [USGS; WVU], Tucker [USGS]. Host: Bombus spp. especially $B$. (Pyrobombus).

Bombus (Psithyrus) fernaldae (Franklin, 1911) ${ }^{\mathbf{N}, \mathbf{P}}$ - Pendleton [OSUC],Tucker [OSUC; USGS; WVU]. Host: Bombus spp.

Bombus (Pyrobombus) bimaculatus Cresson, 1863 - Barbour [USGS], Berkeley [USGS], Cabell [BBSL], Calhoun [AMNH; USGS], Gilmer [AMNH], Greenbrier [USGS], Hampshire [USGS], Hardy [BBSL; UCD; USGS], Jackson [AMNH], Jefferson [USGS], Kanawha [AMNH; BBSL], Lincoln [AMNH; USGS], Logan [USGS], McDowell [USGS], Monongalia [WVU], Monroe [AMNH], Morgan [USGS], Pendleton [USGS], Pleasants [BugGuide], Pocahontas [BBSL; OSUC; USGS], Preston [USGS], Randolph [BBSL; USGS], Roane [AMNH], Summers [BBSL], Tucker [USGS; WVU], Wetzel [RUAC], Wyoming [USGS].

Bombus (Pyrobombus) impatiens Cresson, 1863 - Barbour [USGS], Berkeley [USGS], Cabell [USGS], Calhoun [USGS], Doddridge [BugGuide; USGS], Fayette [BBSL], Gilmer [AMNH], Grant [USGS], Greenbrier [USGS], Hampshire [USGS], Hardy [BBSL; USGS; WVU], Harrison [USGS], Jackson [WVU], Jefferson [BBSL; USGS], Kanawha [AMNH], Lincoln [AMNH; USGS], Mason [USGS], McDowell [USGS], Mineral [USGS], Mingo [WVU], Monongalia [WVU], Monroe [BBSL], Morgan [USGS], Ohio 
[USGS], Pendleton [USGS], Pleasants [USGS], Pocahontas [BBSL; OSUC; USGS], Preston [USGS], Raleigh [BBSL; USGS], Randolph [BBSL; OSUC; USGS], Ritchie [USGS], Roane [AMNH], Summers [BBSL], Tucker [BBSL; USGS; WVU], Tyler [USGS], Upshur [BugGuide], Wayne [UCD], Webster [BugGuide], Wetzel [RUAC], Wirt [BBSL; USGS], Wood [USGS], Wyoming [BBSL].

Bombus (Pyrobombus) perplexus Cresson, 1863 - Berkeley [WVU], Doddridge [BugGuide], Hampshire [BugGuide; USGS], Hardy [WVU], Jefferson [USGS], Kanawha [BBSL], Lincoln [AMNH], Monongalia [WVU], Monroe [AMNH], Pendleton [USGS; WVU; YALE], Pocahontas [WVU], Preston [USGS], Randolph [UCD; OSUC; USGS], Roane [AMNH].

Bombus (Pyrobombus) sandersoni Franklin, $1913^{\mathbf{N}}$ - Greenbrier [USGS], Hampshire [USGS], Hardy [USGS], Monroe [AMNH], Pendleton [USGS], Preston [USGS], Tucker [USGS; WVU].

Bombus (Pyrobombus) vagans Smith, 1854 - See subspecies.

vagans Smith, 1854 - Cabell [BBSL], Calhoun [USGS], Fayette [WVU], Grant [USGS], Greenbrier [USGS], Hampshire [USGS], Hardy [USGS], Jefferson [USGS], McDowell [USGS], Mingo [WVU], Monongalia [NMNH; WVU], Monroe [BBSL], Nicholas [WVU], Pendleton [OSUC; UCD; USGS; WVU], Pocahontas [AMNH; OSUC; USGS; WVU], Preston [USGS; WVU], Raleigh [USGS], Randolph [OSUC; USGS], Ritchie [USGS], Tucker [USGS; WVU], Wayne [UCD], Wetzel [RUAC].

Bombus (Thoracobombus) fervidus (Fabricius, 1798) - Berkeley [USGS], Hampshire [USGS], Jackson [BBSL], Jefferson [USGS], Pendleton [OSUC; USGS], Preston [USGS; WVU], Randolph [OSUC], Upshur [BugGuide].

Bombus (Thoracobombus) pensylvanicus (DeGeer, 1773) - Cabell [BBSL], Hardy [BBSL; UCD], Jefferson [BBSL], Mercer [BBSL], Monongalia [WVU], Pocahontas [BBSL], Preston [WVU], Wayne [UCD]. 
Tribe: Emphorini [2]

Melitoma taurea $\left(\right.$ Say, 1837) ${ }^{\mathbf{o}, \mathbf{s}}$ - Grant [AMNH], Hampshire [USGS], Monongalia [AMNH], Monroe [AMNH], Pleasants [USGS], Ritchie [USGS]. Host: Ipomoea (Convulvulaceae). Ptilothrix bombiformis (Cresson, 1878) ${ }^{\mathbf{o}}$ - Hampshire [USGS]. Host: Hibiscus (Malvaceae).

\section{Tribe: Eucerini [12]}

Cemolobus ipomoeae (Robertson, 1891) ${ }^{\mathbf{2 , O}, \mathrm{S}}$ - Hampshire [USGS], Mineral [WVU]. Host: Ipomoea (Convulvulaceae).

Eucera (Synhalonia) atriventris (Smith, 1854) - Monongalia [WVU], Pleasants [USGS], Ritchie [USGS].

Eucera (Synhalonia) dubitata (Cresson, 1878) ${ }^{\mathbf{S}}$ - Pleasants [USGS].

Eucera (Synhalonia) hamata (Bradley, 1942) - Barbour [WVU], Berkeley [BBSL; USGS], Hampshire [USGS], Jefferson [USGS], Pendleton [USGS], Randolph [USGS], Roane [AMNH], Tucker [USGS].

Melissodes (Eumelissodes) boltoniae Robertson, 1905 - Randolph [USGS].

Melissodes (Eumelissodes) denticulatus Smith, $1854^{\circ}$ - Doddridge [USGS], Hampshire [BBSL; USGS], Hardy [WVU], Harrison [USGS], Kanawha [USGS], Monongalia [WVU], Ohio [BBSL], Wood [USGS]. Host: Asteraceae.

Melissodes (Eumelissodes) druriellus (Kirby, 1802) ${ }^{\mathbf{o}}$ - Cabell [USGS], Doddridge [USGS], Hampshire [USGS], Pocahontas [USGS], Tucker [USGS], Wood [USGS]. Host: Asteraceae.

Melissodes (Eumelissodes) illatus Lovell and Cockerell, $1906^{\mathbf{1 , 0}}$ - Tucker [WVU]. Col. Event: $1 \overbrace{}^{\text {July }} 17^{\text {th }}, 2012 @ 39.09656,-79.36343$; Canaan Valley National Wildlife Refuge on unnamed rd. 7.5 mi. southeast of A Frame rd.; M. McKinney. Host: Asteraceae. Melissodes (Eumelissodes) subillatus LaBerge, $1961^{\circ}$ - Hampshire [USGS], Pendleton [USGS], Tucker [USGS]. Host: Asteraceae. 
Melissodes (Heliomelissodes) desponsus Smith, $1854^{\circ}$ - Hampshire [USGS], Jefferson [USGS], Kanawha [USGS], Mineral [WVU], Monongalia [WVU], Pendleton [USGS], Pleasants [USGS], Randolph [USGS], Tucker [WVU]. Host: Asteraceae.

Melissodes (Melissodes) bimaculatus (Lepeletier, 1825) - See subspecies.

bimaculata (Lepeletier, 1825) - Hardy [WVU], Lincoln [WVU], Mingo [WVU],

Monongalia [WVU], Pendleton [USGS], Pleasants [USGS], Randolph [OSUC], Wood [USGS].

Peponapis (Peponapis) pruinosa (Say, 1837) ${ }^{\mathbf{o}}$ - Hampshire [USGS], Pleasants [USGS], Ritchie [USGS], Wood [USGS]. Host: Cucurbita (Cucurbitaceae).

\section{Tribe: Melectini [1]}

Melecta (Melecta) pacifica Cresson $1878^{\text {S,P }}-$ See subspecies.

atlantica Linsley, 1953 - Grant [USGS], Hardy [USGS]. Host: Anthophora.

\section{Subfamily: Nomadinae [30]}

Tribe: Ammobatoidini [1]

Holcopasites calliopsidis (Linsley, 1943) ${ }^{\mathbf{P}}-$ See subspecies.

calliopsidis (Linsley, 1943) - Kanawha [BBSL], Lewis [WVU], Randolph [USGS], Tucker [USGS]. Host: Calliopsis andreniformis.

Tribe: Epeolini [8]

Epeolus autumnalis Robertson, $1902^{1, \mathbf{P}}-$ Marion [WVU]. Col. Event: $1 \overbrace{}^{\text {Sept. }} 9^{\text {th }}$, $1938 @$ 39.484, -80. 1425; Fairmont; L.H. Taylor. Host: Colletes.

Epeolus bifasciatus Cresson, $1864^{\mathbf{2 , P}}-$ See subspecies.

bifasciatus Cresson, 1864 - Hampshire [BBSL], Mingo [WVU]. Host: Colletes.

Epeolus pusillus Cresson, $1864{ }^{1, \mathbf{P}}$ - Monongalia [WVU]. Col. Event: $1 \overbrace{}^{\Uparrow}$ unknown date @ 39.685, -79.83389; Cheat Neck; J.F. Hall. Host: Colletes.

Epeolus scutellaris Say, $1824^{\mathbf{P}}$ - Preston [WVU], Tucker [WVU]. Host: Colletes.

Triepeolus cressonii (Robertson, 1897) ${ }^{\mathbf{P}}$ - Boone [BBSL], Hardy [USGS]. Host: Colletes. 
Triepeolus donatus (Smith, 1854) ${ }^{2}$ - Berkeley [BBSL]. Host: Colletes.

Triepeolus helianthi (Robertson, 1897) ${ }^{\mathbf{1 , P}}-$ Hampshire [USGS]. Col. Event: $1 \overbrace{}^{\Uparrow}$ Sept. $8^{\text {th }}, 2006$ @ 39.2304, -78.4654; Timber Ridge Camp; S. Droege. Host: Colletes.

Triepeolus lunatus (Say, 1824) ${ }^{\mathbf{2 , P}}$ - Berkeley [BBSL], Monongalia [WVU]. Host: Colletes. Tribe: Nomadini [21]

Nomada articulata Smith, $1854{ }^{\mathbf{P}}$ - Brooke [BBSL], Hampshire [USGS], Nicholas [WVU], Randolph [USGS], Tucker [USGS]. Host: Andrena.

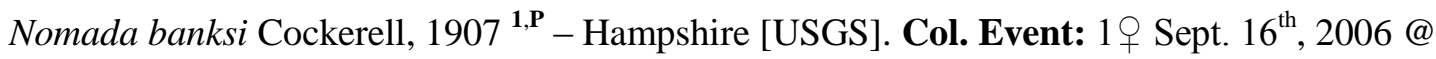
39.3316, -78.4598; Buffalo Gap; S. Droege. Host: Andrena.

Nomada bethunei Cockerell, $1903^{\mathbf{1 , P}}-$ Randolph [USGS]. Col. Event: 1 June 21 $1^{\text {st }}, 2009$ @ 38.852833, -79.529333; Job, White's Run; J. Whitaker. Host: Andrena.

Nomada cressonii Robertson, $1893^{\mathbf{P}}$ - Berkeley [USGS], Brooke [WVU], Hampshire [USGS], Hardy [USGS], Monongalia [WVU], Pendleton [USGS], Pocahontas [USGS], Randolph [USGS], Wayne [USGS]. Host: Andrena.

Nomada crudelis Cresson, $1878^{\text {1,P }}-$ Hampshire [USGS]. Col. Event: 19 July $7^{\text {th }}, 2002 @$ unkown locality; S. Droege. Host: Andrena.

Nomada cuneata (Robertson, 1903) ${ }^{\mathbf{P}}$ - Hampshire [USGS], Pendleton [BBSL]. Host: Andrena. Nomada denticulata Robertson, $1902^{\mathbf{P}}$ - Hampshire [USGS], Hardy [USGS], Kanawha [BBSL]. Host: Andrena.

Nomada depressa Cresson, $1863^{\text {2,P }}$ - Tucker [WVU]. Host: Andrena. Nomada gracilis Cresson, $1863^{\text {2,P }}$ - Tucker [USGS]. Host: Andrena. Nomada illinoensis Robertson, $1900{ }^{1, \mathbf{P}}-$ Berkeley [USGS]. Col. Event: 19 May $7^{\text {th }}, 2005 @$ 39.5187, -77.8412; Whiting's Neck; S. Droege. Host: Andrena.

Nomada imbricata Smith, $1854^{\mathbf{P}}$ - Hampshire [USGS], Jefferson [USGS], Kanawha [BBSL], Monongalia [BBSL], Pendleton [USGS], Randolph [USGS], Ritchie [USGS]. Host: Andrena. 
Nomada lehighensis Cockerell, $1903^{\text {2,P }}$ - Greenbrier [NMNH], Preston [WVU], Tucker [NMNH; WVU]. Host: Andrena.

Nomada lepida Cresson, 1863 - Barbour [BBSL], Wirt [BBSL]. Host: Andrena.

Nomada luteoloides Robertson, 1895 - Hampshire [USGS], Hardy [NMNH; OSUC; USGS], Monongalia [WVU], Morgan [USGS; WVU], Pendleton [BBSL; USGS], Randolph [USGS], Tucker [USGS]. Host: Andrena.

Nomada maculata Cresson, $1863^{\mathbf{P}}$ - Berkeley [USGS], Fayette [BBSL], Grant [USGS],

Hampshire [USGS], Hardy [BBSL; USGS], Jefferson [USGS], Morgan [USGS], Randolph [USGS], Ritchie [USGS], Tucker [USGS]. Host: Andrena.

Nomada ovata (Robertson, 1903) ${ }^{\mathbf{P}}$ - Cabell [BBSL], Kanawha [BBSL], Monongalia [WVU], Wirt [BBSL]. Host: Andrena.

Nomada perplexa Cresson, $1863^{\mathbf{P}}$ - Hampshire [USGS], Mason [BBSL]. Host: Andrena. Nomada pygmaea Cresson, $1863^{\mathbf{P}}$ - Berkeley [USGS], Hampshire [USGS], Hardy [USGS], Jefferson [USGS], Kanawha [USGS], Lincoln [BBSL], Pendleton [BBSL; USGS], Pleasants [USGS], Randolph [BBSL], Tucker [USGS], Wood [USGS]. Host: Andrena. Nomada sulphurata Smith, $1854^{\text {S,P }}$ - Hampshire [USGS], Pleasants [USGS], Randolph [USGS].

Host: Andrena.

Nomada superba Cresson, $1863^{\mathbf{1 , S}, \mathbf{P}}-$ Pleasants [USGS]. Col. Event: 1 April 2 $2^{\text {nd }}, 2012 @$ 39.4119, -81.1975; St. Marys, Middle Island; J. Whitaker. Host: Andrena.

Nomada tiftonensis Cockerell, $1903^{\text {I,P }}-$ Mingo [WVU]. Col. Event: $4 \overbrace{}^{\Uparrow} 1$ July $10^{\text {th }}, 1930 @$ 37.58861, -81.83528; Justice; L. H. Taylor. Host: Andrena.

\section{Subfamily: Xylocopinae [5]}

Tribe: Ceratinini [4]

Ceratina (Zadontomerus) calcarata Robertson, 1900 -Berkeley [USGS], Cabell [USGS], Doddridge [USGS], Grant [USGS], Greenbrier [USGS], Hampshire [USGS], Hardy [USGS], Jackson [AMNH], Jefferson [USGS], Kanawha [AMNH; BBSL], Lincoln 
[USGS], McDowell [USGS], Monongalia [WVU], Morgan [USGS], Ohio [USGS], Pendleton [USGS], Pleasants [USGS], Pocahontas [USGS], Preston [USGS], Randolph [USGS], Ritchie [USGS], Roane [AMNH], Tucker [USGS], Wirt [USGS], Wood [USGS], Wyoming [NMNH].

Ceratina (Zadontomerus) dupla Say, 1837 - Hampshire [USGS], Pleasants [USGS], Wirt [BBSL].

Ceratina (Zadontomerus) mikmaqi Rehan and Sheffield, 2011 - Hampshire [USGS], Jackson [AMNH], Pleasants [USGS], Roane [AMNH].

Ceratina (Zadontomerus) strenua Smith, 1879 - Berkeley [USGS], Boone [BBSL], Cabell [USGS], Grant [USGS], Hampshire [USGS], Hardy [AMNH; USGS], Jefferson [USGS], Kanawha [AMNH], Lincoln [USGS], McDowell [USGS], Monongalia [WVU], Ohio [USGS], Pendleton [USGS], Pleasants [BBSL; USGS], Raleigh [USGS], Randolph [USGS], Ritchie [USGS], Roane [AMNH], Tucker [USGS], Wood [USGS], Wyoming [USGS].

Tribe: Xylocopini [1] Xylocopa (Xylocopoides) virginica (Linnaeus, 1771) - See subspecies. virginica (Linnaeus, 1771) - Berkeley [USGS], Cabell [USGS], Calhoun [AMNH; USGS], Doddridge [USGS], Gilmer [AMNH], Grant [USGS], Greenbrier [USGS], Hampshire [USGS], Hardy [AMNH; USGS], Jefferson [USGS], Kanawha [BBSL], Lincoln [USGS], Logan [USGS], McDowell [USGS], Mingo [WVU], Monongalia [WVU], Morgan [USGS], Ohio [USGS], Pendleton [WVU; USGS], Pleasants [USGS], Preston [USGS], Ritchie [USGS], Tucker [WVU], Wayne [USGS].

\section{FAMILY COLLETIDAE [15]}

\section{Subfamily: Colletinae [9]}

Tribe: N/A [9] 
Colletes americanus Cresson, 1868 - Hampshire [USGS], Monongalia [WVU], Randolph [WVU].

Colletes compactus Cresson, $1868^{\mathbf{o}}$ - See subspecies. Host: Asteraceae.

compactus Cresson, 1868 - Hampshire [USGS], Monongalia [WVU], Pleasants [USGS], Randolph [USGS], Ritchie [USGS].

Colletes eulophi Robertson, $1891^{\mathrm{S}}$ - Recorded by Mitchell (1960); no specific locality. Notes: Its northern replacement $C$. kincaidii Cockerell, 1898, may occur at higher elevations.

Colletes inaequalis Say, 1837 - Jefferson [BBSL; USGS], Lincoln [USGS], Logan [USGS], Pleasants [USGS], Randolph [USGS], Tucker [USGS; WVU], Wayne [USGS], Wood [USGS].

Colletes latitarsis Robertson, $1891^{\text {o }}$ - Hardy [WVU], Jefferson [CAES], Mingo [WVU], Pendleton [WVU]. Host: Physalis (Solanaceae)

Colletes nudus Robertson, $1898^{2}$ - Monongalia [WVU], Randolph [WVU].

Colletes productus Robertson, $1891^{\mathbf{1 , 0}}-$ Randolph [WVU]. Col. Event: 19 July $16^{\text {th }}, 1936 @$ 38.71278, -79.97889; Huttonsville; L. H. Taylor. Host: Lyonia (Ericaceae)

Colletes simulans Cresson, $1868^{\circ}-$ See subspecies. Host: Asteraceae. armatus Patton, 1879 - Hampshire [USGS], Pendleton [WVU], Randolph [USGS], Ritchie [USGS], Tucker [USGS].

Colletes thoracicus Smith, 1853 - Hampshire [USGS], Kanawha [BBSL], Logan [BBSL], Marshall [BugGuide], Nicholas [WVU], Pendleton [USGS], Wayne [USGS].

\section{Subfamily: Hylaeinae [6]}

Tribe: Hylaeini [6]

Hylaeus (Hylaeus) annulatus (Linnaeus, 1758) ${ }^{\mathbf{N}}-$ Grant [USGS], Greenbrier [USGS], Pocahontas [BBSL], Randolph [USGS], Tucker [AMNH]. 
Hylaeus (Hylaeus) leptocephalus (Morowitz, 1871) ${ }^{\mathbf{E}}$ - Harrison [USGS], Randolph [USGS], Ritchie [USGS].

Hylaeus (Hylaeus) mesillae (Cockerell, 1896) - See subspecies.

cressonii (Cockerell, 1907) - Grant [USGS], Hampshire [USGS], Pleasants [USGS],

Preston [AMNH; USGS], Raleigh [AMNH; USGS], Randolph [USGS], Ritchie [USGS], Tucker [USGS].

Hylaeus (Prosopis) affinis (Smith, 1853) - Barbour [BBSL], Boone [BBSL], Grant [USGS],

Hampshire [BBSL; USGS], Kanawha [BBSL], Mineral [BBSL], Monongalia [WVU], Morgan [BBSL], Pleasants [USGS], Randolph [USGS], Ritchie [USGS], Tucker [USGS], Wetzel [BBSL].

Hylaeus (Prosopis) illinoisensis (Robertson, 1896) ${ }^{2}$ - Grant [USGS], Mineral [USGS]. Hylaeus (Prosopis) modestus Say, 1837 - See subspecies.

modestus Say, 1837 - Barbour [BugGuide], Berkeley [USGS], Doddridge [USGS], Greenbrier [BBSL; USGS], Hampshire [USGS], Hancock [BBSL], Hardy [NMNH; USGS], Jackson [BBSL], Mason [BBSL], McDowell [USGS], Mineral [NMNH], Mingo [BBSL], Monongalia [BBSL], Monroe [AMNH], Pendleton [USGS], Pleasants [USGS], Pocahontas [BBSL], Preston [AMNH; USGS], Raleigh [WVU], Randolph [USGS; BBSL], Ritchie [USGS], Taylor [BBSL], Tucker [WVU], Upshur [BBSL], Wetzel [BBSL], Wirt [USGS].

\section{FAMILY HALICTIDAE [82]}

\section{Subfamily: Halictinae [82]}

Tribe: Augochlorini [5]

Augochlora (Augochlora) pura (Say, 1837) - Barbour [USGS], Berkeley [BBSL; USGS], Cabell [BBSL], Calhoun [AMNH], Doddridge [USGS], Gilmer [AMNH], Grant [USGS], 
Greenbrier [BBSL; USGS], Hampshire [BBSL; USGS], Hancock [BBSL], Hardy [BBSL; NMNH; USGS], Harrison [USGS], Jefferson [USGS], Kanawha [AMNH; BBSL], Lewis [AMNH], Lincoln [AMNH], Logan [BBSL; USGS], Mason [BBSL], McDowell [USGS], Mineral [USGS], Monroe [AMNH], Morgan [USGS], Ohio [BBSL; USGS], Pendleton [AMNH; BBSL; USGS], Pleasants [USGS], Pocahontas [AMNH; CUIC], Preston [BBSL; USGS], Raleigh [BBSL], Randolph [USGS], Ritchie [USGS], Roane [AMNH], Summers [AMNH], Tucker [USGS; WVU], Tyler [USGS], Wirt [BBSL; USGS], Wood [USGS], Wyoming [USGS].

Augochlorella aurata (Smith, 1853) - Berkeley [USGS], Calhoun [AMNH], Doddridge [USGS], Fayette [BBSL], Grant [USGS], Greenbrier [AMNH; BBSL; USGS], Hampshire [BBSL; USGS], Hardy [NMNH; USGS], Harrison [USGS], Jefferson [USGS], Kanawha [BBSL], McDowell [USGS], Mineral [USGS], Monongalia [BBSL], Nicholas [BBSL], Pendleton [AMNH; BBSL; USGS], Pocahontas [BBSL], Preston [USGS], Raleigh [USGS], Randolph [USGS], Ritchie [USGS], Roane [AMNH; BBSL], Tucker [AMNH; BBSL; USGS], Tyler [USGS], Wood [USGS], Wyoming [BBSL].

Augochlorella persimilis (Viereck, 1910) ${ }^{\mathbf{S}}$ - Berkeley [USGS], Gran [BBSL], Hampshire [BBSL; USGS], Mineral [USGS]. Augochloropsis (Paraugochloropsis) metallica (Fabricius, 1793) - Berkeley [USGS], Calhoun [AMNH], Doddridge [USGS], Gilmer [AMNH], Grant [USGS], Hampshire [USGS], Hardy [BBSL; USGS; WVU], Jackson [AMNH], Jefferson [USGS], Kanawha [BBSL], Monongalia [WVU], Morgan [WVU], Pendleton [BBSL; USGS; WVU], Pleasants [USGS], Pocahontas [BBSL], Preston [USGS], Randolph [USGS], Ritchie [BBSL; USGS], Roane [AMNH], Tucker [USGS; WVU], Tyler [USGS].

Augochloropsis (Paraugochloropsis) sumptuosa (Smith, 1853) ${ }^{\mathbf{1 , S}}$ - Hampshire [USGS]. Col. Event: July 30 $0^{\text {th }}, 2005 @ 39.2489,-78.5256$; Burgundy Center for Wildlife Studies; collector unknown. 
Tribe: Halictini [77]

Agapostemon (Agapostemon) sericeus (Forster, 1771) - Barbour [WVU], Doddridge [BBSL, USGS], Greenbrier [WVU], Hampshire [BBSL; USGS], Hardy [USGS], Kanawha [BBSL], Mingo [WVU], Pendleton [USGS], Ritchie [USGS].

Agapostemon (Agapostemon) splendens (Lepeletier, 1841) ${ }^{2}$ - Hampshire [USGS], Mingo [WVU].

Agapostemon (Agapostemon) texanus (Cresson, 1872) - Berkeley [USGS], Grant [USGS], Hampshire [USGS], Jefferson [USGS].

Agapostemon (Agapostemon) virescens (Fabricius, 1775) - Barbour [USGS], Berkeley [USGS], Cabell [BBSL], Grant [USGS; WVU], Hampshire [USGS], Hardy [USGS; WVU], Jefferson [USGS; WVU], Ohio [USGS], Pendleton [USGS], Pleasants [USGS], Pocahontas [BBSL], Randolph [USGS], Tucker [USGS], Wood [USGS]. Halictus (Nealictus) parallelus Say, 1837 - Greenbrier [BBSL], Hampshire [USGS], Jefferson [USGS], Ritchie [USGS].

Halictus (Odontalictus) ligatus Say, 1837 - Berkeley [USGS], Boone [BBSL], Doddridge [USGS], Fayette [BBSL], Grant [USGS], Greenbrier [BBSL; USGS], Hampshire [BBSL; USGS], Hardy [USGS; WVU], Harrison [USGS], Jackson [AMNH; BBSL], Jefferson [USGS], Monongalia [WVU], Monroe [BBSL], Morgan [BBSL], Ohio [USGS], Pendleton [AMNH; USGS], Pleasants [USGS], Preston [USGS], Raleigh [USGS], Randolph [USGS], Ritchie [USGS], Tucker [AMNH; USGS; WVU], Tyler [USGS], Wood [USGS], Wyoming [USGS].

Halictus (Protohalictus) rubicundus (Christ, 1791) - Berkeley [USGS], Brooke [WVU], Calhoun [AMNH], Gilmer [AMNH], Greenbrier [AMNH], Hampshire [USGS], Hardy [USGS], Jackson [AMNH], Jefferson [USGS], Kanawha [AMNH; BBSL], Lincoln [USGS], Monongalia [WVU], Ohio [USGS], Pocahontas [AMNH], Preston [USGS; WVU], Randolph [USGS], Roane [AMNH], Tucker [WVU], Wetzel [RUAC], Wood [USGS]. 
Halictus (Seladonia) confusus Smith, 1853 - See subspecies.

confusus Smith, 1853 - Berkeley [USGS], Grant [USGS], Greenbrier [AMNH],

Hampshire [USGS], Hardy [NMNH; USGS], Jackson [AMNH], Jefferson [USGS], Ohio [USGS], Pendleton [AMNH; USGS], Pocahontas [BBSL], Preston [BBSL; USGS],

Randolph [USGS], Ritchie v, Roane [USGS], Tucker [USGS; WVU], Wetzel [RUAC],

Wood [USGS]

Lasioglossum (Dialictus) abanci (Crawford, 1932) ${ }^{2}$ - Hampshire [USGS].

Lasioglossum (Dialictus) admirandum (Sandhouse, 1924) ${ }^{2}$ - Hampshire [USGS], Hardy

[NMNH], Morgan [NMNH], Pleasants [USGS].

Lasioglossum (Dialictus) albipenne (Robertson, 1890) - Hampshire [USGS], Hardy [USGS].

Lasioglossum (Dialictus) anomalum (Robertson, 1892) ${ }^{\mathbf{1}}$ - Randolph [USGS]. Col. Event: 1 ㅇ

May $5^{\text {th }}, 2010 @ 38.8528,-79.5293$; Jobe, White’s Run Rd.; J. Whitaker.

Lasioglossum (Dialictus) apocyni (Mitchell, 1960) ${ }^{\text {S }}$ - Berkeley [USGS], Calhoun [AMNH],

Gilmer [AMNH], Hampshire [USGS], Hardy [USGS], McDowell [USGS], Pendleton

[USGS], Randolph [USGS], Ritchie [USGS], Roane [AMNH], Tucker [USGS], Wood [USGS].

Lasioglossum (Dialictus) asteris (Mitchell, 1960) ${ }^{2}$ - Hardy [NMNH], Randolph [USGS].

Lasioglossum (Dialictus) bruneri (Crawford, 1902) - Grant [USGS], Hampshire [BBSL; USGS],

Hardy [NMNH; USGS], Jefferson [USGS], Monongalia [WVU], Pleasants [USGS],

Ritchie [USGS].

Lasioglossum (Dialictus) callidum (Sandhouse, 1924) - Hampshire [USGS], Hardy [NMNH;

USGS], Jefferson [USGS], McDowell [USGS], Pendleton [USGS], Randolph [USGS],

Ritchie [USGS], Tucker [USGS], Tyler [USGS].

Lasioglossum (Dialictus) cattellae (Ellis, 1913) - Hardy [USGS], Pendleton [USGS], Randolph [USGS]. 
Lasioglossum (Dialictus) coeruleum (Robertson, 1893) - Grant [USGS], Hardy [USGS], Kanawha [BBSL], Pendleton [AMNH; USGS], Pocahontas [BBSL], Ritchie [USGS], Wyoming [AMNH].

Lasioglossum (Dialictus) cressonii (Robertson, 1890) - Berkeley [USGS], Grant [USGS], Hampshire [USGS], Hardy [USGS], Jefferson [USGS], McDowell [USGS], Pendleton [USGS], Pocahontas [USGS], Preston [USGS], Randolph [USGS], Ritchie [USGS], Tucker [USGS; WVU].

Lasioglossum (Dialictus) curculum Gibbs, $2011^{\mathbf{1 , S}}-$ Hardy [USGS]. Col. Event: 19 June $7^{\text {th }}$, 2007 @ 38.9166, -78.8166; 3 mi. northeast of Mathias; D. Smith.

Lasioglossum (Dialictus) ephialtum Gibbs, 2010 - Hardy [USGS], McDowell [USGS], Pendleton [USGS], Pleasants [USGS], Preston [USGS], Randolph [USGS], Ritchie [USGS], Wood [USGS].

Lasioglossum (Dialictus) fattigi (Mitchell, 1960) - Doddridge [USGS], Hampshire [USGS], Hardy [NMNH], Pendleton [USGS], Preston [USGS], Randolph [USGS], Tucker [USGS].

Lasioglossum (Dialictus) gotham Gibbs, 2011 - Morgan [USGS], Pendleton [USGS], Wood [USGS].

Lasioglossum (Dialictus) heterognathum (Mitchell, 1960) - Hampshire [USGS], Preston [USGS], Tucker [WVU].

Lasioglossum (Dialictus) hitchensi Gibbs, 2012 - Doddridge [USGS], Grant [USGS], Hampshire [USGS], Hardy [NMNH; USGS], Jackson [AMNH], Jefferson [USGS], Lincoln [AMNH; USGS], Morgan [USGS], Ohio [USGS], Pendleton [USGS], Pleasants [USGS], Preston [USGS], Randolph [USGS], Ritchie [USGS], Roane [USGS], Tyler [USGS], Wood [USGS]. 
Lasioglossum (Dialictus) illinoense (Robertson, 1892) - Berkeley [USGS], Hampshire [BBSL; USGS], Hardy [USGS], Jefferson [USGS], Randolph [USGS], Tucker [USGS], Wood [USGS], Wyoming [BBSL].

Lasioglossum (Dialictus) imitatum (Smith, 1853) - Berkeley [BBSL; USGS], Calhoun [AMNH], Doddridge [USGS], Gilmer [AMNH], Grant [USGS; WVU], Hampshire [USGS], Hardy [NMNH; USGS], Jackson [AMNH; BBSL], Jefferson [USGS], Kanawha [BBSL; AMNH], Lincoln [USGS], Logan [BBSL], McDowell [USGS], Minderal [USGS], Monongalia [BBSL; WVU], Ohio [BBSL; USGS], Pendleton AMNH; BBSL; USGS], Pleasants [USGS], Preston [AMNH; USGS], Randolph [USGS], Ritchie [USGS], Roane [AMNH], Tucker [USGS; WVU], Wood [USGS].

Lasioglossum (Dialictus) laevissimum (Smith, 1853) - Berkeley [USGS], Hampshire [USGS], Hardy [NMNH; USGS], Jefferson [USGS], Pendleton [USGS].

Lasioglossum (Dialictus) leucocomum (Lovell, 1908) - Randolph [USGS], Roane [AMNH]. Lasioglossum (Dialictus) lineatulum (Crawford, 1906) - Berkeley [BBSL], Grant [USGS], Hampshire [USGS], Hardy [USGS], Mason [BBSL], Pendelton [AMNH; USGS], Pocahontas [USGS], Preston [USGS], Randolph [USGS], Ritchie [BBSL], Tucker [USGS].

Lasioglossum (Dialictus) nigroviride (Graenicher, 1911) ${ }^{\mathbf{N}}$ - Doddridge [USGS], Hardy [USGS], Kanawha [BBSL], Morgan [USGS], Pocahontas [AMNH; BBSL], Randolph [USGS], Ritchie [USGS], Tucker [WVU].

Lasioglossum (Dialictus) oblongum (Lovell, 1905) - Berkeley [USGS], Hampshire [USGS], Hardy [NMNH], Tucker [USGS].

Lasioglossum (Dialictus) obscurum (Robertson, 1892) - Cabell [USGS], Grant [USGS], Hampshire [USGS], Hardy [NMNH; USGS], Lincoln [USGS], Pendleton [USGS], Pleasants [USGS], Pocahontas [USGS], Randolph [USGS]. 
Lasioglossum (Dialictus) oceanicum (Cockerell, 1916) - Grant [USGS], Hampshire [USGS], Jefferson [USGS], Pendleton [USGS], Pocahontas [BBSL; USGS], Randolph [BBSL; USGS], Tucker [AMNH; USGS], Wayne [BBSL].

Lasioglossum (Dialictus) paradmirandum (Knerer and Atwood, 1966) ${ }^{\mathbf{N}}-$ Jackson $_{\text {[AMNH], }}$ Lincoln [AMNH].

Lasioglossum (Dialictus) perpunctatum (Ellis, 1913) - Hampshire [USGS], Hardy [NMNH], Tucker [USGS].

Lasioglossum (Dialictus) pilosum (Smith, 1853) - Berkeley [USGS], Grant [USGS], Hampshire [USGS], Hardy [USGS], Jefferson [USGS], Morgan [BBSL], Randolph [USGS], Tucker [USGS; WVU].

Lasioglossum (Dialictus) planatum (Lovell, 1905) - Doddridge [USGS], Hardy [USGS], Pocahontas [USGS], Randolph [USGS], Ritchie [USGS].

Lasioglossum (Dialictus) platyparium (Robertson, 1895) - Hampshire [USGS], Jefferson [USGS], Pendleton [USGS], Randolph [USGS].

Lasioglossum (Dialictus) pruinosum (Robertson, 1892) ${ }^{\mathbf{1}}$ - Hampshire [USGS]. Col. Event: 1 ㅇ July $11^{\text {th }}, 2002 @ 39.2425,-78.6137$; County rd. 7/1 3.8 mi. south of Rt. 50; S. Droege. Lasioglossum (Dialictus) rozeni Gibbs, 2011 - Hampshire [USGS], Hardy [USGS], Randolph [USGS].

Lasioglossum (Dialictus) simplex (Robertson, 1901) ${ }^{\mathbf{1}}$ - Hampshire [USGS]. Col. Event: Sept. $20^{\text {th }}, 2002 @ 39.3348,-78.458$; Buffalo Gap Community Camp; S. Droege.

Lasioglossum (Dialictus) smilacinae (Robertson, 1899) - Hardy [USGS], Pleasants [USGS].

Lasioglossum (Dialictus) subviridatum (Cockerell, 1938) - Grant [USGS], Greenbrier [USGS], Hampshire [USGS], Hardy [USGS], Pleasants [USGS].

Lasioglossum (Dialictus) tegulare s.l. (Robertson, 1890) - Berkeley [USGS], Hampshire [USGS], Hardy [NMNH; USGS], Jefferson [USGS], Kanawha [AMH], Pendleton [USGS], Tucker [WVU]. 
Lasioglossum (Dialictus) tenax (Sandhouse, 1924) ${ }^{\mathbf{1}}-$ Hardy [USGS]. Col. Event: 1 \% , Aug. $11^{\text {th }}$, 2006 @ 38.9166, -78.8166; 3 mi. northeast of Mathias; D. Smith.

Lasioglossum (Dialictus) timothyi Gibbs, 2010 - Hardy [USGS], Wood [USGS].

Lasioglossum (Dialictus) trigeminum Gibbs, 2011 - Hampshire [USGS], Hardy [USGS], Jackson [AMNH], Jefferson [USGS], Randolph [USGS].

Lasioglossum (Dialictus) versans (Lovell, 1905) ${ }^{\mathbf{N}}$ - Grant [USGS], Hampshire [USGS], Hardy

[USGS], Monroe [AMNH], Morgan [USGS], Pendleton [USGS], Preston [USGS],

Randolph [USGS], Ritchie [USGS], Tucker [AMNH; USGS].

Lasioglossum (Dialictus) versatum (Robertson, 1902) - Berkeley [USGS], Calhoun [AMNH],

Doddridge [USGS], Greenbrier [USGS], Hampshire [USGS], Hardy [NMNH; USGS],

Jackson [AMNH], Jefferson [USGS], Kanawha [AMNH; BBSL], Lewis [AMNH],

Lincoln [USGS], McDowell [USGS], Mineral [USGS], Monongalia [BBSL], Pendleton

[USGS], Pleasants [USGS], Pocahontas [BBSL], Preston [USGS], Randolph [USGS],

Ritchie [USGS], Roane [AMNH], Tucker [USGS], Tyler [USGS], Wood [USGS].

Lasioglossum (Dialictus) weemsi (Mitchell, 1960) - Hamphire [USGS], Hardy [USGS], Jackson

[AMNH], Pleasants [USGS], Tucker [USGS].

Lasioglossum (Dialictus) zephyrum (Smith, 1853) - Berkeley [USGS], Hampshire [USGS],

Hardy [NMNH; USGS], Jefferson [USGS], Lincoln [BBSL], Randolph [USGS], Roane [AMNH], Wayne [USGS].

Lasioglossum (Evylaeus) cinctipes (Provancher, 1888) - Fayette [WVU], Morgan [WVU],

Pocahontas [BBSL], Roane [AMNH].

Lasioglossum (Hemihalictus) birkmanni (Crawford, 1906) - Barbour [USGS], Berkeley [USGS],

Hampshire [USGS], Hardy [NMNH; USGS], Kanawha [BBSL], McDowell [USGS],

Pendleton [USGS], Randolph [USGS].

Lasioglossum (Hemihalictus) foxii (Robertson, 1895) - Berkeley [USGS], Calhoun [AMNH],

Hampshire [USGS], Hardy [USGS], Jefferson [CUIC], McDowell [USGS], Mingo 
[USGS], Pendleton [USGS], Pleasants [USGS], Preston [USGS], Raleigh [CUIC; AMNH], Randolph [USGS], Ritchie [USGS], Roane [AMNH].

Lasioglossum (Hemihalictus) inconditum (Cockerell, 1916) ${ }^{\mathbf{1}}$ - Kanawha [BBSL]. Col. Event: 1 古 June $10^{\text {th }}, 1993 @ 38.405903,-81.659927$; Guthrie; collector unknown.

Lasioglossum (Hemihalictus) pectinatum (Robertson, 1890) ${ }^{\mathbf{1 , 0}}$ - Hampshire [USGS]. Col.

Event: $1 \delta^{\lambda}$ August $8^{\text {th }}, 2006 @ 39.2304,-78.4654$; Timber Ridge Camp; S. Droege.

Host: Solanaceae.

Lasioglossum (Hemihalictus) pectorale (Smith, 1853) - Berkeley [USGS], Hampshire [BBSL; USGS], Hardy [NMNH; USGS], Tucker [WVU].

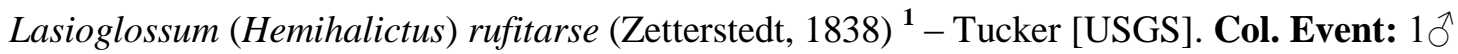

Sept. $2^{\text {nd }}, 2008$ @ 39.0815,-79.4625; Davis, Canaan Loop Rd.; J. Whitaker.

Lasioglossum (Lasioglossum) acuminatum McGinley, 1986 - Berkeley [BBSL], Hampshire

[USGS], Hardy [BBSL; USGS].

Lasioglossum (Lasioglossum) athabascense (Sandhouse, 1933) ${ }^{\mathbf{N}}$ - Pocahontas [USGS], Randolph [USGS].

Lasioglossum (Lasioglossum) coriaceum (Smith, 1853) - Berkeley [USGS], Grant [USGS;

WVU], Greenbrier [USGS], Hampshire [BBSL; USGS], Hardy [NMNH; USGS], Jefferson [BBSL; USGS], McDowell [USGS], Monongalia [WVU], Monroe [AMNH], Nicholas [WVU], Pendleton [BBSL; USGS], Pocahontas [USGS], Preston [USGS; WVU], Randolph [USGS], Ritchie [USGS], Tucker [USGS; WVU], Wood [USGS]. Lasioglossum (Lasioglossum) forbesii (Robertson, 1890) - Grant [USGS], Hampshire [USGS], Hardy [USGS].

Lasioglossum (Lasioglossum) fuscipenne (Smith, 1853) - Boone [BBSL], Hampshire [BBSL; USGS], Hardy [USGS], Jackson [AMNH], Jefferson [USGS], Kanawha [BBSL], Logan [USGS], Mason [BBSL]. 
Lasioglossum (Leuchalictus) leucozonium (Schrank, 1781) ${ }^{\mathbf{E}}$ - Berkeley [USGS], Grant [USGS], Hampshire [USGS], Hardy [USGS], Jefferson [USGS], McDowell [USGS], Pendleton [AMNH, USGS], Preston [USGS], Randolph [USGS], Tucker [USGS].

Lasioglossum (Leuchalictus) zonulum (Smith, 1848) ${ }^{\mathbf{2 , N}}$ - Hampshire [USGS].

Lasioglossum (Sphecodogastra) oenotherae (Stevens, 1920) ${ }^{\text {I,o }}$ - Jackson [WVU]. Col. Event:

$2 q$ June $29^{\text {th }}, 1930$ @ locality unknown; L. H. Taylor. Host: Onagraceae.

Lasioglossum (Sphecodogastra) quebecense (Crawford, 1907) - Berkeley [USGS], Grant

[USGS], Greenbrier [USGS], Hampshire [USGS], Hardy [USGS], Jefferson [USGS],

Ohio [USGS], Pendleton [BBSL; USGS], Raleigh [BBSL], Randolph [BBSL; USGS],

Tucker [USGS], Wyoming [USGS].

Lasioglossum (Sphecodogastra) truncatum (Robertson, 1901) - Berkeley [USGS], Hampshire

[USGS], Hardy [USGS], Jefferson [USGS], Morgan [USGS], Randolph [USGS], Tucker [USGS].

Sphecodes aroniae Mitchell, $1960^{\mathbf{1 , P}}-$ Tucker [WVU]. Col. Event: 19 May $17^{\text {th }}, 2012 @$

39.13286, -79.44238; Canaan Valley National Wildlife Refuge, Camp 70 rd. 1.2 mi. east of highway 32; M. McKinney. Host: Halictinae.

Sphecodes confertus Say, $1837^{\text {2,P }}$ - Barbour [WVU], Lincoln [WVU].

Sphecodes coronus Mitchell, $1956^{\text {2,P }}$ - Hardy [NMNH; USGS], Tucker [AMNH].

Sphecodes cressonii (Robertson, 1903) ${ }^{\mathbf{1 , P}}-$ Berkeley [BBSL]. Col. Event: 1? June 24 ${ }^{\text {th }}, 1986$ @ 39.509342, -78.167307; Sleepy Creek Hunting Area; collector unknown.

Sphecodes davisii Robertson, $1897^{\text {1,P }}-$ Hampshire [BBSL]. Col. Event: 1 ? Oct. $7^{\text {th }}, 1986 @$ 39.430600, -78.713221; Grace; collector unknown. Host: Halictinae.

Sphecodes mandibularis Cresson, $1872^{\mathbf{P}}$ - Calhoun [AMNH], Hardy [BBSL], Kanawha [BBSL].

Host: Halictinae.

Sphecodes minor Robertson, $1898^{\mathbf{1 , P}}-$ Roane [AMNH]. Col. Event: $1 \overbrace{}^{\Uparrow}$ June $11^{\text {th }}, 2011 @$ 38.59488, -81.40226; 2.9 mi. south of Walton; A. Payne. Host: Halictinae. 
Sphecodes townesi Mitchell, $1956^{\mathbf{1}}$ - Hardy [USGS]. Col. Event: $1 q$ June $9^{\text {th }}, 2006$ locality unknown; D. Smith.

\section{FAMILY MEGACHILIDAE [61]}

\section{Subfamily: Megachilinae [61]}

Tribe: Anthidiini [9]

Anthidiellum (Loyolanthidium) notatum (Latreille, 1809) - See subspecies.

notatum (Latreille, 1809) - Hampshire [USGS], Hardy [WVU], Jefferson [WVU], Morgan [WVU].

Anthidium (Anthidium) maculifrons ${ }^{\mathbf{1 , S}}-$ Barbour [BBSL]. Col. Event: 19 Aug. $10^{\text {th }}, 1950 @$ 39.091086, -79.985717; Calhoun; collector unknown.

Anthidium (Anthidium) manicatum (Linnaeus, 1758) ${ }^{\mathbf{E}}-$ See subspecies.

manicatum (Linnaeus, 1758) - Hampshire [BBSL; USGS], Jefferson [USGS], Monongalia [WVU].

Anthidium (Proanthidium) oblongatum (Illiger, 1806) ${ }^{\mathbf{E}}$ - See subspecies.

oblongatum (Illiger, 1806) - Berkeley [USGS], Calhoun [AMNH; USGS], Greenbrier [AMNH; BBSL], Hampshire [USGS], Jackson [AMNH; BBSL], Jefferson [USGS], Roane [AMNH; BBSL], Tucker [WVU].

Paranthidium (Paranthidium) jugatorium (Say, 1824) ${ }^{2}$ - See subspecies. jugatorium (Say, 1824) - Hampshire [USGS], Monongalia [WVU].

Stelis (Dolichostelis) louisae (Cockerell, 1911) ${ }^{\mathbf{1 , P}}-$ Mingo [WVU]. Col. Event: 19 July $10^{\text {th }}$, 1930 @ 37.58861, -81.83528; Justice; L. H. Taylor. Host: Osmiini and Anthidiini. Stelis (Stelis) labiata (Provancher, 1888) 1,P - Randolph [BBSL]. Col. Event: 1ô June ??, ???? @ 38.609499, -79.939861; Cheat Mountain; collector unknown. Host: Osmiini and Anthidiini. 
Stelis (Stelis) lateralis Cresson, $1864^{\mathbf{P}}$ - Jefferson [USGS], Randolph [USGS], Wyoming

[WVU]. Host: Osmiini and Anthidiini.

Stelis (Stelis) nitida Cresson, $1878^{\mathbf{1 , P}}-$ Hampshire [USGS]. Col. Event: 19 May 29 $9^{\text {th }}, 2004 @$ 39.34575, -78.40269; County road 45/1 2 mi. southwest of Good; S. Droege. Host:

Osmiini and Anthidiini.

Tribe: Megachilini [24]

Coelioxys (Boreocoelioxys) octodentata Say, $1824{ }^{\mathbf{P}}$ - Hampshire [USGS], Jeffrson [USGS],

Mingo [WVU], Monongalia [WVU], Morgan [WVU], Tucker [USGS]. Host: Megachile.

Coelioxys (Boreocoelioxys) porterae Cockerell, $1900{ }^{\mathbf{P}}$ - Randolph [USGS], Roane [AMNH],

Tucker [USGS, WVU]. Host: Megachile.

Coelioxys (Boreocoelioxys) rufitarsis Smith, $1854^{\mathbf{1 , P}}-$ Greenbrier [USGS]. Col. Event: $1 \overbrace{}^{\Uparrow}$ Sept.

$5^{\text {th }}, 2010$ @ 37.9752, -80.3617; Spring Creek, Rt. 13; J. Whitaker. Host: Megachile.

Coelioxys (Boreocoelioxys) sayi Robertson, $1897^{\mathbf{P}}$ - Boone [WVU], Hampshire [USGS],

Jefferson [USGS], Mingo [WVU], Monongalia [WVU], Pendleton [USGS], Pleasants

[USGS], Pocahontas [USGS; WVU], Ritchie [USGS], Tucker [USGS; WVU]. Host:

Megachile.

Coelioxys (Cyrtocoelioxys) modesta Smith, $1854^{\text {1,P }}-$ Lincoln [WVU]. Col. Event: 19 July $4^{\text {th }}$,

1930 @38.27861, -82.10278; Hamlin; L. H. Taylor. Host: Megachile.

Coelioxys (Synocoelioxys) alternata Say, $1837^{\mathbf{1 , P}}-$ Tucker [USGS]. Col. Event: $1 \overbrace{}^{\Uparrow}$ Sept $3^{\text {rd }}$, 2008 @ 39.00445, -79.4855; Hendricks, Back Hollow Rd.; J. Whitaker. Host:

Megachile.

Coelioxys (Synocoelioxys) hunteri Crawford, $1914^{\mathbf{1}}$ - Monongalia [WVU]. Col. Event: 19 Aug. $13^{\text {th }}, ? ? ? ? @ 39.630723,-79.955482$; Morgantown; collector unknown. Host: Megachile. Megachile (Callomegachile) sculpturalis Smith, $1853^{\mathbf{E}}$ - Hampshire [USGS], Monongalia [WVU], Roane [AMNH]. 
Megachile (Chelostomoides) campanulae (Robertson, 1903) - Hampshire [USGS], Hardy [USGS], Jefferson [USGS], Mason [WVU], Monongalia [WVU], Pendleton [USGS], Ritchie [USGS].

Megachile (Chelostomoides) exilis Cresson, $1872^{\mathrm{S}}$ - See subspecies. parexilis (Mitchell, 1937) - Calhoun [AMNH], Gilmer [AMNH], Hampshire [USGS], Hardy [USGS], Jackson [AMNH], Jefferson [USGS], Kanawha [AMNH], Roane $[\mathrm{AMNH}]$.

Megachile (Eutricharaea) rotundata (Fabricius, 1787) ${ }^{\mathbf{E}}$ - Gilmer [AMNH], Greenbrier [AMNH], Hampshire [BBSL; USGS], Hardy [WVU], Jackson [AMNH], Jefferson [USGS], Lincoln [AMNH], Roane [AMNH], Tucker [USGS].

Megachile (Leptorachis) petulans Cresson, $1878^{2, S}$ - Doddridge [USGS], Monongalia [WVU]. Megachile (Litomegachile) brevis Say, 1837 - Hampshire [BBSL; USGS], Hardy [USGS; WVU], Jefferson [USGS], Monongalia [WVU], Pendleton [USGS], Randolph [USGS], Roane $[\mathrm{AMNH}]$.

Megachile (Litomegachile) mendica Cresson, 1878 - Berkeley [USGS], Doddridge [USGS], Grant [WVU], Greenbrier [AMNH; USGS], Hampshire [USGS], Hardy [NMNH; USGS; WVU], Harrison [USGS], Jackson [AMNH], Jefferson [USGS; WVU], Kanawha [AMNH], Logan [WVU], Marshal [WVU], Mason [WVU], Mingo [WVU], Monongalia [WVU], Monroe [AMNH], Nicholas [WVU], Pendleton [USGS; WVU], Pleasants [USGS], Pocahontas [USGS], Randolph [USGS], Ritchie [USGS], Roane [AMNH], Tucker [USGS; WVU], Tyler [USGS], Wood [USGS].

Megachile (Litomegachile) texana Cresson, 1878 - Hampshire [USGS], Hardy [WVU], Jackson [AMNH].

Megachile (Megachile) inermis Provancher, $1888^{\mathbf{N}}$ - Pendleton [USGS], Pocahontas [BBSL; AMNH], Randolph [USGS], Tucker [USGS; WVU]. 
Megachile (Megachile) montivaga Cresson, $1878^{1}$ - Mason [WVU]. Col. Event: $1 \bigcirc^{\Uparrow}$ Aug. $8^{\text {th }}$, 1971 @ locality unknown; L. Butler.

Megachile (Megachile) relativa Cresson, 1878 - Grant [USGS], Greenbrier [USGS], Hampshire [USGS], Monongalia [WVU], Morgan [WVU], Pendleton [USGS], Preston [USGS], Raleigh [WVU], Randolph [USGS], Tucker [WVU].

Megachile (Sayapis) frugalis Cresson, 1872 - See subspecies.

frugalis Cresson, 1872 - Hampshire [USGS], Monongalia [WVU].

Megachile (Sayapis) inimica Cresson, 1872 - See subspecies.

sayi Cresson, 1878 - Grant [USGS; WVU], Hampshire [USGS], Hardy [USGS], Mason [BBSL; WVU], Monongalia [WVU], Preston [WVU], Tucker [WVU].

Megachile (Sayapis) pugnata Say, $1837^{\mathbf{0}}$ - See subspecies.

pugnata Say, 1837 - Berkeley [USGS], Grant [USGS], Hampshire [BBSL; USGS], Hardy [USGS], Jefferson [USGS], Mineral [USGS], Tucker [USGS]. Host: Asteraceae. Megachile (Xanthosarus) gemula Cresson, 1878 - See subspecies.

gemula Cresson, 1878 - Calhoun [AMNH], Grant [USGS], Hampshire [USGS], Hardy [USGS], Mason [WVU], Mingo [WVU], Monongalia [WVU], Pendleton [WVU], Pocahontas [USGS], Preston [USGS], Randolph [USGS], Roane [AMNH], Tucker [USGS; WVU].

Megachile (Xanthosarus) latimanus Say, 1823 - Berkeley [BBSL], Hampshire [USGS], Hardy [WVU], Mason [WV], Mercer [WVU], Monongalia [WVU], Randolph [USGS; WVU], Summers [WVU], Tucker [USGS; WVU].

Megachile (Xanthosarus) mucida Cresson, $1878^{\mathbf{I , 2}}-$ Preston [USGS]. Col. Event: $2 q$ June $11^{\text {th }}$, 2010 @ 39.4584, -79.5236; Terra Alta, Camp Galilee; J. Whitaker.

\section{Tribe: Osmiini [28]}

Chelostoma (Prochelostoma) philadelphi (Robertson, 1891) - Hampshire [USGS], Monongalia [WVU], Roane [AMNH]. 
Heriades (Neotrypetes) carinata Cresson, 1864 - Calhoun [AMNH], Gilmer [AMNH],

Greenbrier [BBSL], Hampshire [USGS], Jackson [AMNH], Randolph [USGS]. Note:

There is an additional record from an unknown locality labelled only "Cheat River."

Heriades (Neotrypetes) variolosa (Cresson, 1872) - Berkeley [USGS], Greenbrier [BBSL],

Hampshire [USGS], Hardy [USGS], Jefferson [USGS].

Hoplitis (Alcidamea) pilosifrons (Cresson, 1864) - Hampshire [USGS], Hardy [USGS], Jefferson

[USGS], McDowell [USGS], Pendleton [USGS], Pleasants [USGS], Randolph [USGS],

Tucker [USGS].

Hoplitis (Alcidamea) producta (Cresson, 1864) - See subspecies.

producta (Cresson, 1864) - Hampshire [USGS], Hardy [USGS], Jefferson [USGS],

McDowell [USGS], Randolph [USGS], Roane [AMNH], Tucker [USGS].

Hoplitis (Alcidamea) spoliata (Provancher, 1888) - Hampshire [USGS], Jefferson [USGS],

McDowell [USGS], Nicholas [WVU], Pendleton [USGS], Pocahontas [USGS], Randolph

[USGS], Tucker [USGS].

Hoplitis (Alcidamea) truncata (Cresson, 1878) - See subspecies.

truncata (Cresson, 1878) - Gilmer [AMNH], Hampshire [USGS], Hardy [USGS].

Hoplitis (Hoplitis) anthocopoides (Schenck, 1853) ${ }^{\mathbf{E}}$ - Hampshire [USGS], Randolph [USGS].

Hoplitis (Robertsonella) simplex (Cresson, 1864) ${ }^{\mathbf{2}, 0}$ - Cabell [USGS], Hampshire [USGS]. Host:

Hydrophylloideae (Boraginaceae).

Osmia (Diceratosmia) conjuncta Cresson, 1864 - Berkeley [USGS], Hampshire [USGS], Hardy

[USGS], Jefferson [USGS], Lincoln [USGS], Monongalia [WVU], Pendleton [USGS],

Pleasants [USGS].

Osmia (Diceratosmia) subfasciata Cresson, $1872^{2}$ - Hardy [USGS], Tucker [USGS].

Osmia (Helicosmia) caerulescens (Linnaeus, 1758) - See subspecies.

caerulescens (Linnaeus, 1758) - Hardy [USGS], Monongalia [WVU], Randolph [BBSL;

USGS]. 
Osmia (Helicosmia) chalybae Smith, 1853 - Greenbrier [USGS], Hampshire [BBSL; USS]. Osmia (Helicosmia) georgica Cresson, 1878 - Berkeley [USGS], Calhoun [AMNH], Grant [USGS], Hampshire [USGS], Hardy [USGS], Jefferson [USGS], Kanawha [AMNH], Lincoln [USGS], McDowell [USGS], Mineral [BBSL], Morgan [USGS], Pendleton [BBSL; USGS], Pleasants [USGS], Preston [BBSL], Putnam [BBSL], Raleigh [WVU], Randolph [USGS], Ritchie [USGS], Tucker [USGS], Wood [USGS], Wyoming [BBSL; USGS].

Osmia (Helicosmia) texana Cresson, 1872 - Grant [WVU], Greenbrier [USGS], Hardy [WVU]. Osmia (Melanosmia) albiventris Cresson, $1864^{2}$ - Greenbrier [USGS], Hampshire [USGS]. Osmia (Melanosmia) atriventris Cresson, 1864 - Berkeley [USGS], Hampshire [USGS], Hardy [USGS], Jefferson [USGS], Kanawha [BBSL], Logan [USGS], Mason [BBSL], McDowell [USGS], Mineral [BBSL], Morgan [USGS], Nicholas [BBSL], Pendleton [USGS], Pleasants [USGS], Randolph [USGS], Ritchie [USGS], Roane [AMNH], Tucker [USS; WVU], Wetzel [RUAC], Wood [USGS].

Osmia (Melanosmia) bucephala Cresson, 1864 - Berkeley [USGS], Calhoun [USGS], Gilmer [AMNH], Greenbrier [USGS], Hampshire [USGS], Hardy [USGS], Jefferson [USGS], McDowell [USGS], Ohio [USGS], Pendleton [BBSL; USGS], Pleasants [USGS], Ritchie [USGS], Roane [AMNH], Tucker [USGS; WVU], Wood [USGS]. Osmia (Melanosmia) collinsiae Robertson, 1905 - Berkeley [USGS], Hampshire [USGS], Hardy [USGS], Jefferson [USGS], Lincoln [USGS], McDowell [USGS], Pendleton [USGS], Pleasants [USGS], Randolph [USGS], Wood [USGS]. Osmia (Melanosmia) distincta Cresson, 1864 - Hampshire [USGS], Hardy [USGS], Mineral [WVU], Pendleton [USGS], Pocahontas [USGS]. Osmia (Melanosmia) felti Cockerell, $1911^{\mathbf{N}}$ - Hampshire [USGS], Hardy [USGS]. Osmia (Melanosmia) pumila Cresson, 1864 - Berkeley [BBSL; USGS], Cabell [USGS], Grant [USGS], Greenbrier [USGS], Hampshire [USGS], Hardy [BBSL; USGS], Jefferson 
[USGS], Kanawha [BBSL], Lincoln [USGS], McDowell [USGS], Morgan [USGS], Pendleton [BBSL; USGS], Pleasants [USGS], Pocahontas [USGS; WVU], Randolph [USGS], Ritchie [USGS], Tucker [USGS], Wayne [USGS], Wetzel [RUAC], Wood [USGS].

Osmia (Melanosmia) sandhouseae Mitchell, $1927^{\mathbf{1}}$ - Hampshire [USGS]. Col. Event: 1 ㅇ May $28^{\text {th }}, 2001 @ 39.3348,-78.4576$; Capon Bridge; S. Droege.

Osmia (Melanosmia) simillima Smith, $1853^{\mathbf{1}}-$ Randolph [USGS]. Col. Event: 19 July $17^{\text {th }}$, 2009 @38.852833,-79.529333; Job, White’s Run Rd.; J. Whitaker.

Osmia (Melanosmia) virga Sandhouse, $1939^{\circ}$ - Hardy [USGS], Pendleton [BBSL]. Host:

Vaccinium (Ericaceae).

Osmia (Osmia) cornifrons (Radoszkowski, 1887) ${ }^{\mathbf{E}}$ - Berkeley [USGS], Hampshire [USGS], Hardy [USGS], Jefferson [USGS], Monongalia [WVU], Ohio [USGS], Pendleton [USGS], Tucker [USGS].

Osmia (Osmia) lignaria Say, 1837 - See subspecies.

lignaria Say, 1837 - Berkeley [BBSL], Grant [WVU], Greenbrier [BBSL], Hampshire [AMNH; USGS], Hardy [CUIC; USGS], Jefferson [BBSL], Kanawha [BBSL], Lincoln [USGS], Logan [USGS], Mercer [AMNH], Monongalia [WVU], Pendleton [BBSL; USGS], Pocahontas [AMNH; BBSL], Preston [WVU], Randolph [USGS], Tucker [USGS], Wayne [BBSL], Wetzel [RUAC], Wood [USGS].

Osmia (Osmia) taurus Smith, $1873^{\mathbf{E}}$ - Berkeley [USGS], Calhoun [USGS], Grant [USGS], Greenbrier [USGS], Hampshire [USGS], Hardy [USGS], Jefferson [USGS], Lincoln [USGS], Morgan [USGS], Pendleton [USGS], Pleasants [USGS], Randolph [USGS], Ritchie [USGS], Tucker [USGS], Wood [USGS].

FAMILY MELITTIDAE [3]

\section{Subfamily: Melittinae [3]}


Tribe: Melittini [1]

Melitta (Cilissa) eickworti Snelling and Stage, $1995^{\circ}$ - Hampshire [USGS], Hardy [USGS].

Hosts: Vaccinium stamineum (Ericaceae).

\section{Tribe: Macropodini [2]}

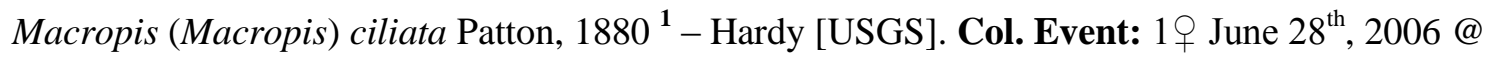
locality unknown; D. Smith.

Macropis (Macropis) nuda (Provancher, 1888) ${ }^{\mathbf{1 , N}}$ - Monongalia [WVU]. Col. Event: $1 q$ July $16^{\text {th }}, 1937$ @ 39.173836, -79.107506; Greenland Gap; L. H. Taylor.

\section{Discussion}

Prior to this research little or no information was available regarding the bee fauna of West Virginia. Here we have listed 301 species, and given relevant information regarding their natural history. The diversity found in the Apidae, Megachilidae, Andrenidae, and Halictidae was quite similar, though halictid specimens made up the more than $50 \%$ of the collection records (Figure 2). We note that there are likely many more records to be found, both from undetermined specimens currently deposited in West Virginia University's Insect and Arthropod Museum, and from future statewide surveys. Donovall and van Engelsdorp (2010) reported 371 species from the neighboring state of Pennsylvania. Given the large number of species reported from a nearby region, we believe many species remain unrecorded from the state. Additionally, collecting in the state prior to the last seven years has been minimal, with recent collections made primarily by Ansel Payne, Matthew McKinney, and Sam Droege. Historical information mostly originates from some early-mid 1900s collections done at West Virginia University, and some historical specimens found at the National Museum of Natural History.

With concerns over the collapse of Apis mellifera populations, conservation of native bee communities has become extensively studied in recent years (Stockstad 2007). Before a 
conservation plan can be developed for a given region, it is important that baseline information regarding the diversity of the region's bees be understood. It is our hope that researchers interested in the conservation of native bees, and those who are concerned with exotic species will find this list particularly useful in pursuing their goals.

Several important and noteworthy records are present in the list, including the southernmost range locality for Bombus fernaldae. Several species are known only from historical collections, and have not been collected in the past twenty years within West Virginia's borders. These species include Andrena confederata from 1976, Andrena mariae from 1966, Andrena erythrogaster (no recorded date), Perdita octomaculata from 1977, Psuedopanurgus andrenoides from 1976, Habropoda laboriosa from 1921, Bombus ashtoni from 1931, Epeolus autumnalis from 1938, Epeolus pusillus (no recorded date), Nomada tiftonensis from 1930, Colletes productus from 1936, Lasioglossum inconditum from 1993, Lasioglossum oenotherae from 1930, Sphecodes confertus from 1977, Sphecodes coronus from 1977, Sphecodes cressonii from 1986, Sphecodes davisii from 1986, Anthidium maculifrons from 1950, Stelis louisae from 1930, Stelis labiata (no recorded date), Coelioxys modesta from 1930, Coelioxys hunteri (no recorded date), Megachile montivaga from 1971, Macropis nuda from 1937. Given the lack of collecting done in much of West Virginia, it is difficult to ascertain if these species are extirpated, in decline, or simply have not been detected by entomologists in the state. Further investigations as to the status of these species would be particularly beneficial to our knowledge of West Virginia's bee fauna and to future conservation efforts.

\section{Acknowledgements}

We wish to thank the many collection managers who assisted us in this research including Bob Davidson (CMNH), Haley Cartmell (VMNH), Terry Griswold (BBSL), Sean Brady (NMNH), Sean Brady (NMNH), Larry Gall (YALE) and numerous others. Jason Gibbs of 
Cornell University graciously provided much help and guidance on the challenging genus

Lasioglossum. Sam Droege shared data in the USGS bee database from his fieldwork in the state; he identified a large number of new state records based on specimens collected by Jane Whitaker, and they generously made these available for this study. I must recognize my collaborators, John Ascher and Ansel Payne, who contributed both writing and editing to this chapter. Without the contributions of these and many other individuals, this work would not have been possible.

\section{References Cited}

Albu, V. 1997. Notes of the sesiid fauna of southwestern West Virginia. J. Lep. Soc. 51(3): 249256.

Amrine, J.W., and L. Butler. 1978. Annotated list of the mosquitoes of West Virginia. Mosq. News 38: 101-104.

Arthropod Easy Capture. http://sourceforge.net/p/arthropodeasy. Version: 1.34. Date: February $18,2013$.

Ascher, J.S., and J. Pickering. 2016. Discover Life bee species guide and world checklist (Hymenoptera: Apoidea: Anthophila). Draft 45. 18 May 2016. http://www.discoverlife.org/mp/20q?guide=Apoidea_species\&flags=HAS:

Bartomeus, I., J. S. Ascher, J. Gibbs, B. N. Danforth, D. L. Wagner, S. M. Hedtke, and R. Winfree. 2013. Historical changes in northeastern United States bee pollinators related to shared ecological traits. Proc. Natl. Acad. Sci. 110(12): 4656-4660.

Blauch, D.S. 1975. Toward a natural delineation of the area known as the Southern Appalachian Highlands. Castanea 40 (3): 197-201. 
Braun, L.E. 1950. Deciduous forests of eastern North America. Philadelphia: The Blakiston Co., Philadelphia, PA.

Brooks, M. and W.C. Legg. 1942. Swainson's Warbler in Nicholas County, West Virginia. The Auk. 59(1): 76-86.

Colla, S. R., J. S. Ascher, M. Arduser, J. Cane, M. Deyrup, S. Droege, J. Gibbs, T. Griswold, H. G. Hall, J. Neff, R. P. Jean, M. G. Rightmyer, C. Sheffield, M. Veit, and A. Wolf. 2012. Documenting persistence of most eastern North American bee species (Hymenoptera: Apoidea: Anthophila) to 1990-2009. J. Kans. Entomol. Soc. 85(1): 14-22.

Donovall, L.R., and D. van Engelsdorp. 2010. A checklist of the bees (Hymenoptera: Apoidea) of Pennsylvania. J. Kans. Entomol. Soc. 83(1): 7-24.

Drees, B. M., and L. Butler. 1978. Rhopalocera of West Virginia. J. Lep. Soc. 32(3): 198-206.

Drees, B. M., L. Butler, and L. L. Pechuman. 1980. Horse flies and deer flies of West Virginia: an illustrated key (Diptera, Tabanidae). Agr. For. Exp. Stn. Bull. 674. WV University. 67 p.

Engel, M.S. 2005. Family-Group names for bees (Hymenoptera; Apoidea). Am. Mus. Novit. 3476: 1-33.

Fisher, J. E., and D. C. Tarter. 1988. Records of nymphal Stenonema (Ephemeroptera: Heptageniidae) from West Virginia. Entomol. News 99: 30-32.

Gibbs, J. S., L. Packer, S. Dumesh, and B. N. Danforth. 2013. Revision and reclassification of Lasioglossum (Evylaeus), L. (Hemihalictus) and L. (Sphecodogastra) in eastern North America (Hymenoptera: Apoidea: Halictidae). Zootaxa 3672:1-117.

Glover, J.B., and D.C. Tarter. 1990. The Leptoceridae (Trichoptera) of West Virginia. Entomol. News 101(1): 35-38. 
Hoshiba, H., and M. Sasaki. 2008. Perspectives of multi-modal contribution of honeybee resources to our life. Entomol. Res. 38(2008): S15-S21.

Hurd, P.D., Jr. 1979. Superfamily Apoidea in Catalog of Hymenoptera in America North of Mexico vol. 2. eds. K.V. Krombein, P.D. Hurd Jr., D.R. Smith, and B.D. Burks. Smithsonian Institution Press: Washington, D.C. pp. 1741-2209.

Joy, J. E., C. A. Allman, and B. T. Dowell. 1994. Mosquitoes of West Virginia: an update. J. Am. Mosq. Contr. Assoc. 10(1): 115-118.

Kearns, C. A., D. W. Inouye, and N. M. Waser. 2008. Endangered mutualisms: The conservation of plant-pollinator interactions. Annu. Rev. Ecol. Sys. 29(1998): 83-112.

Klein A. -M., B. E. Vassiere, J. H. Cane, I. Steffan-Dewenter, S. A. Cunningham, C. Kremen, and T. Tscharntke. 2007. Importance of pollinators in changing landscapes for world crops. Proc. R. Soc. B. 274(1608): 303-313.

Mader, E., M. Shepherd, M.Vaughan, S. H. Black, and G. LeBuhn. 2011. Attracting native pollinators: protecting North America's bees and butterflies. Storey Publishing, North Adams, MA.

Michener, C.D. 2007. The Bees of the World, 2nd edition. Johns Hopkins University Press: Baltimore, Maryland. 953 pp.

Mitchell, T.B. 1960. Bees of the eastern United States, volume 1. N. C. Agric. Exp. Stn. Tech. Bull. 141: 1-538.

Mitchell, T.B. 1962. Bees of the eastern United States, volume 2. N. C. Agric. Exp. Stn. Tech. Bull. 152: 1-557.

Moure, J.S., and P.D. Hurd Jr. 1987. An Annotated catalog of the halictid bees of the Western Hemisphere. Smithson. Inst. Press: Washington, D.C. 405 pp. 
Pauley, T.K. 2008. The Appalachian inferno: Historical causes for the disjunct distribution of Plethodon nettingi (Cheat Mountain Salamander). Northeast. Nat. 15(4): 595-606.

Palmer, M.D., E.S. Bernhardt, W.H. Schlesinger, K.N. Eshelman, E. Foufoula-Georgiou, M. S. Hendryx, A. D. Lemly, G. E. Likens, O. L. Louks, M. E. Power, P. S. White, and P. R. Wilcock. 2010. Mountaintop mining consequences. Science 327: 148-149.

Sedivy, C., S. Dorn, and A. Müller. 2013. Molecular phylogeny of the bee genus Hoplitis (Megachilidae: Osmiini) - how does nesting biology affect biogeography? Zool. J. Linn. Soc. 167: $28-42$.

Schuh, R.T., S. Hewson-Smith, and J.S. Ascher. 2010. Specimen databases: A case study in entomology using web-based software. Am. Entomol. 56 (4): 206-216.

Scott, V. L., J. S. Ascher, T. L. Griswold, and C. R. Nufio. 2011. The Bees of Colorado (Hymenoptera: Apoidea: Anthophila). Natural History Inventory of Colorado 23, 100 pp.

Stokstad, E. 2007. The case of the empty hives. Science 316: 970-972.

Strausbaugh, P. D., and E. L. Core. 1978. The flora of West Virginia. Seneca Books, Morgantown, WV.

Tarter, D. C., and R. F. Kirchner. 1975. List of the stoneflies (Plecoptera) of West Virginia. Entomol. News 91: 49-53.

Tarter, D. C., and C. H. Nelson. 2006. A revised checklist of the stoneflies (Plecoptera) of West Virginia (USA). Proc. Entomol. Soc. of Washington. 108(2): 429-442.

Tarter, D. C., and C. H. Nelson. 2010. New state, county, and drainage basin records of West Virginia (USA) stoneflies (Plecoptera). Entomol. News 121(2): 159-162.

Tarter, D. C., J. E. Bailey, M. J. Whitman. 2013. New distribution records of fishflies (Megaloptera: Corydalidae) for West Virginia, USA. Entomol. News. 123(4): 278-284. 
Tarter, D. C. 1990. A checklist of the caddisflies of caddisflies (Trichoptera) of West Virginia. Entomol. News 10(4): 236-245.

The White House. 2015. National strategy to promote the health of honey bees and other pollinators. Pollinator Health Task Force.

Wheeler, A.G., T.J. Henry, and T.L. 1983. An annotated list of the Miridae of West Virginia (Hemiptera-Heteroptera). Trans. Am. Entomol. Soc. 109(1): 127-158.

Wolf, A.T. and J.S. Ascher. 2008. Bees of Wisconsin (Hymenoptera: Apoidea: Anthophila). Gr. Lak. Entomol. 41 (3-4): 129-168. 
Table 1. Museum collection codes used in the species list.

\begin{tabular}{lll}
\hline Code & Institution Name & Location \\
\hline AMNH & American Museum of Natural History & New York, NY, USA \\
BBSL & Bee Biology and Systematics Laboratory & Logan, Utah, USA \\
BMEC & Bohart Museum of Entomology Collection & Davis, CA, USA \\
CAES & Connecticut Agricultural Experiment Station & New Haven, CT, USA \\
CUIC & Cornell University Insect Collection & Ithaca, NY, USA \\
NMNH & National Museum of Natural History & Washington, DC, USA \\
OSUC & Ohio State University & Columbus, OH, USA \\
RUAC & Rutgers University Arthropod Collection & New Brunswick, NJ, USA \\
UNHP & University of New Hampshire & Durham, NH, USA \\
USGS & United States Geologic Survey & Beltsville, MD, USA \\
WVU & West Virginia University & Morgantown, WV, USA \\
\hline
\end{tabular}


Table 2. List of problematic taxa. The species listed below could not be confirmed as part of the West Virginia fauna. These include: 1) published records based on misidentifications, 2) unverifiable identifications, and 3) accidental occurrences.

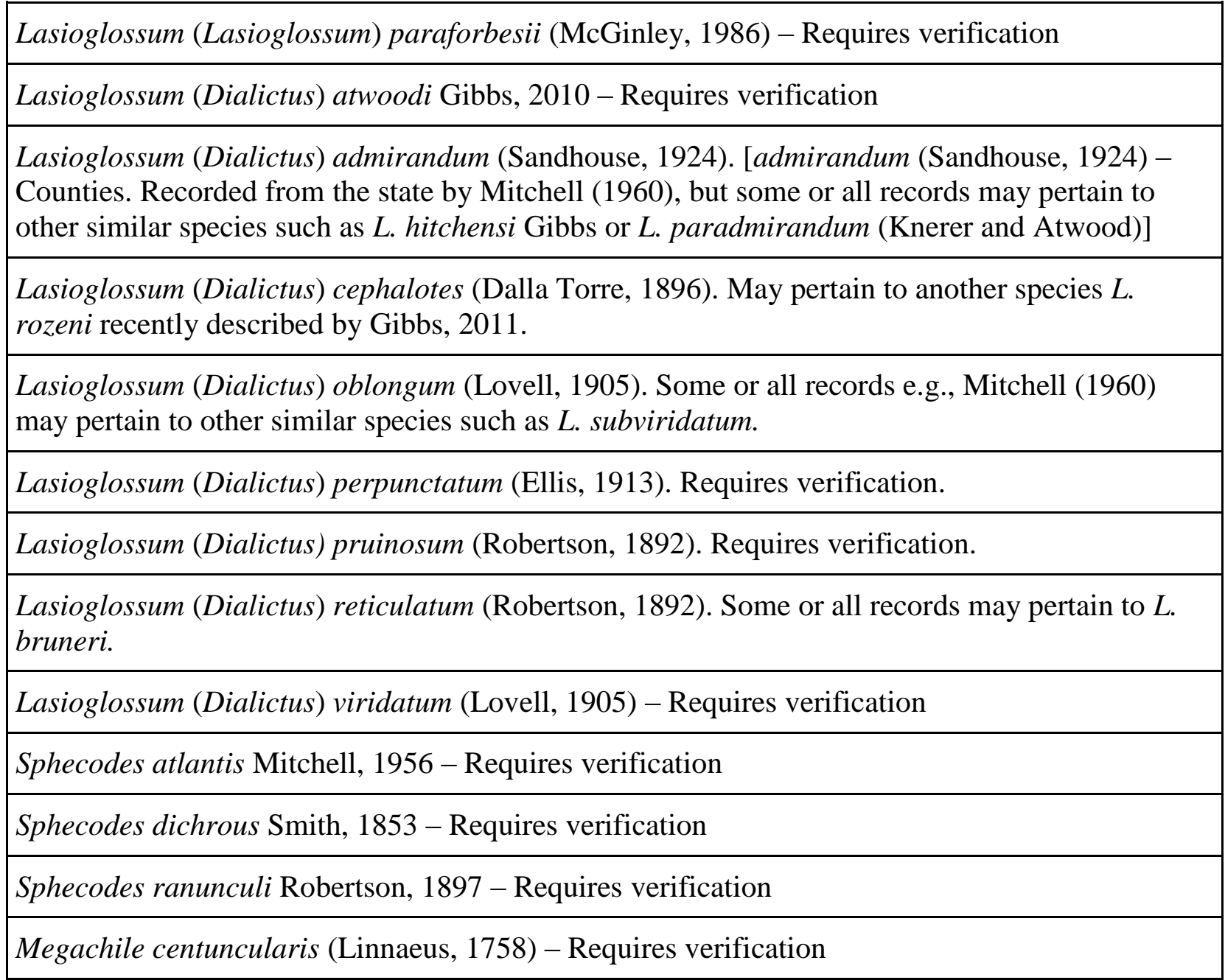




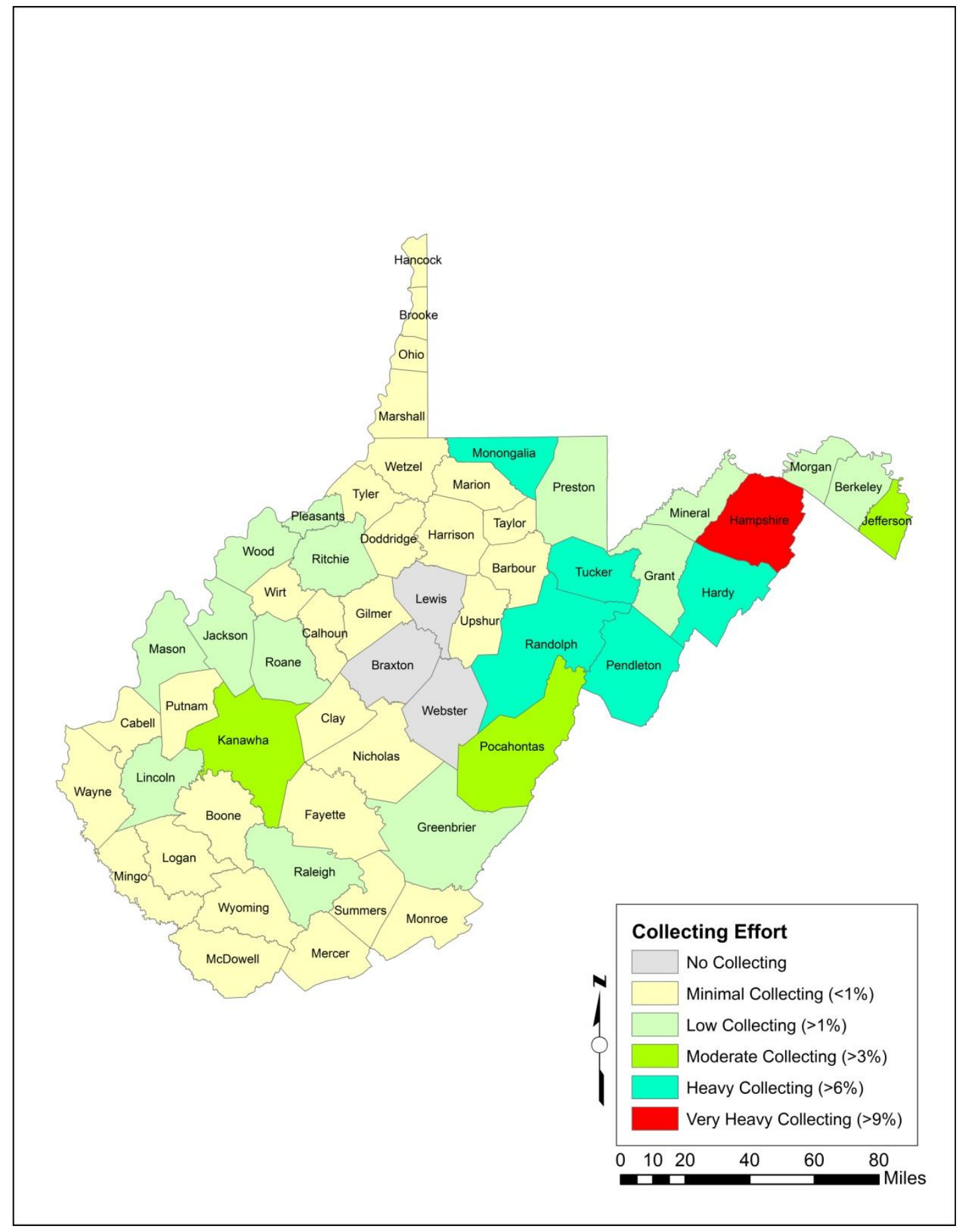

Figure 1. Collecting effort throughout West Virginia depicted as the percentage of collecting trips made in each county. 

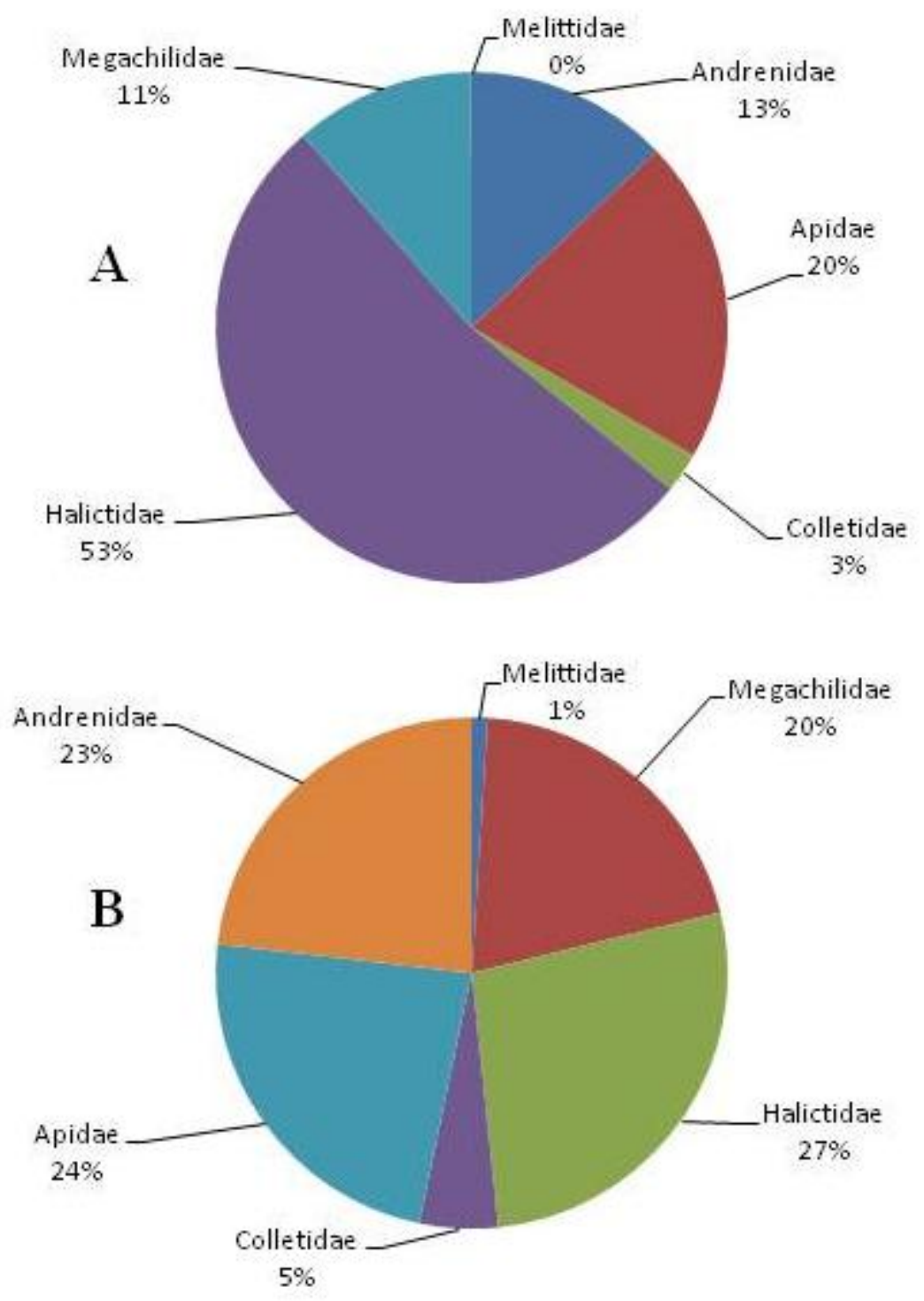

Figure 2. Pie charts showing percentage of specimens from each family included in the list (A) and percentage of species recorded from each family (B). 


\title{
CHAPTER 3: DETERMINATION OF SPATIAL DEPENDENCE AMONGST PAN TRAPS IN BEE SAMPLING PLANS
}

\author{
Abstract \\ Several methods of active and passive sampling exist for collecting bees (Hymenoptera: \\ Apoidea), but pan traps (a.k.a., bee bowls) are possibly the most utilized. Pan trap popularity for \\ bee collecting is largely due to two factors. First, pan traps are time efficient as they may collect \\ for multiple days with little effort required from the researcher. Second, pan traps remove \\ collector bias from experimental design, though there is likely some bias created by the traps \\ themselves. When comparing pan trap samples using traditional statistical methods, it is \\ important that the assumption of independence among samples is met. We investigated spatial \\ dependence in pan traps at four sites in West Virginia. At each site a regular grid of pan traps \\ was laid out, with each point in the grid having two colors of fluorescent trap (i.e., blue and \\ yellow). These trap pairs were separated by $10 \mathrm{~m}$, with a minimum of 100 points in the grid. Five \\ points within the grid were chosen at random, and additional traps were placed out from those \\ five points in each cardinal direction, at a distance of $2 \mathrm{~m}$ and $4 \mathrm{~m}$ from the center point. Traps \\ collected bees for five consecutive days and bee counts were recorded for each point in the grid, \\ and for each trap color. Trap count data was analyzed using semivariograms to determine the \\ range of spatial dependency in trap catches for each of yellow and blue traps, and for yellow and \\ blue traps combined. The largest range value found across the four sites and pan trap colors was \\ $17.3 \mathrm{~m}$ for blue traps. From this, we recommend a conservative distance of $18 \mathrm{~m}$ between pan \\ traps to obtain spatially independent samples. This research is useful for optimizing sampling \\ plans for bee inventory and monitoring programs, and for enhancing ecological study design \\ when independent samples are necessary.
}

Keywords: sampling, pan traps, bee bowls, Hymenoptera, geostatistics, semivariogram. 


\section{Introduction}

Currently, there are several methods utilized for documenting bee community structure in ecological studies. These methods may be active (i.e., transect walks, observation plots) or passive (i.e., malaise trapping, pan trapping). Transect walks are typically done by sweeping all vegetation along a specified transect, and are normalized across sites by restricting the duration of collection time or area in which bees are collected (Moeller 2006). Because transect walks require netting skills, experienced researchers will have greater collecting success than inexperienced technicians (Westphal et al. 2008), indicating sampling bias. Observation plots are standardized by time, where a researcher sits in a given plot and notes all species visiting the plot. This method is useful when killing bees is undesirable, but identifying some problematic taxa, such as Lasioglossum (Dialictus), to species without a pinned specimen may be difficult or impossible. Malaise trapping works by capturing bees in flight. Malaise traps take advantage of a bee's behavior to fly upwards when confronted with an obstacle (i.e., negative geotaxis). As the bee flies upwards it is guided into a collecting head with a killing agent. Kalhorn et al. (2003) used malaise traps to describe bee communities in Appalachian shale barrens. Bartholomew and Prowell (2005) found malaise trap catch to be similar in the number of species caught when compared to pan traps, but fewer total individuals were collected in the malaise traps. Pan traps are bowls or small cups filled with a water/detergent mixture; detergent is used to break the surface tension of the water. It is common for pan traps to be painted white, blue, or yellow due to the attractiveness of these colors to bees (Kirk 1984, Leong and Thorp 1999, Toler et al. 2005). Fluorescent paints have been shown to be more effective in catching bees than nonfluorescent paints (Bartholomew and Prowell 2005). Other colors have been used, such as green (Stephen and Rao 2005), and these colors may be more effective in some unique ecological 
situations such as when that color represents a particularly enticing foraging opportunity to a species of bee. One disadvantage of pan traps is non-target insect kill, a problem shared with malaise traps (Vega et al. 1990). Additionally, pan traps may be biased toward smaller bodied bees, such as halictids (Hymenoptera: Halictidae) (Westphal et al. 2008, Wilson et al. 2008).

While all of these methods may be utilized, current research leans towards the use of pan traps and transect sweeping. Pan traps in particular are very popular in bee sampling protocols due the passive manner in which they sample and their ease of deployment. Many previous studies have used pan traps as a primary means of collecting (Moeller 2006, Brosi et al. 2007, Westphal et al. 2008), and several recent studies have been concerned with the effectiveness of pan trapping when compared to other collecting methods (Cane et al. 2000, Bartholomew and Prowell 2005, Roulston et al. 2007, Grundel et al. 2011). Currently, only Droege et al. (2010) have investigated the spatial relationship between pan traps to maximize trap capture. They did this using variance-mean ratio, Green's index, and the runs test. These methods are limited as they do not take into consideration distance and direction of among sample points and function on the assumption that samples are independent of one-another. A better method which may be used to fully determine spatial dependency among samples is geostatistics. Geostatistical analysis has the advantage of starting from an assumption that points (pan traps in this case) are spatially dependent upon one-another, rather than an assumption of independence found in traditional statistics. Additionally, geostatistics considers both the distance and direction of sample points relative to each other.

Spatial dependence occurs when sample points which are closer together are more likely to have similar values than sample points which are farther apart (Isaaks and Srivastava 1989). In sampling programs, it is important to know the minimum distance at which each sampling point 
is independent of each other sampling point. Gathering independent samples from pan traps is useful for two reasons. First, to perform traditional statistics on trap capture data the assumption of independence must be met. That is, a trap at one location should not impact the capture rate of a nearby trap. This is important when answering common ecological inquiries such as comparing abundance data at two different sites. Second, by making traps independent of one another, capture rate will increase. The distance in which trap captures are independent of one another can be determined using semivariogram in geostatistics. One feature of the semivariogram is the range, which is the distance at which the semivariance reaches its peak. In other words, the range is the distance at which the spatial relationship of a measured variable begins to break down (Krajewski and Gibbs 2001). The range of the semivariogram may then be used to optimize sampling plans for obtaining independent samples. Park and Tollefson (2006) used this technique to get independent samples of northern and western corn rootworm eggs in Iowa cornfields.

This study will be the first to apply geostatistics to solve a common problem in bee sampling protocols. The goal of this study is to standardize bee sampling methodology when using pan traps so that bee diversity and abundance data collected by different researchers may be comparable. This work will be valuable to melittologists utilizing pan traps the primary sampling method in their research.

\section{Materials and Methods}

Experimental Sites

Four sites were chosen to conduct trapping experiments; two sites on an agricultural research farm (Ag. Farm W and Ag. Farm E), one site on an organic research farm (Org. Farm), and one site in a mowed field in a rural area (Philippi). The Ag. Farm W (39.660669 N, - 
79.901111 E) and Ag. Farm E (39.660974 N, -79.905635 E) sites were located on an agricultural research farm in Morgantown, WV. Both of these sites were in mowed plots surrounded by unmowed fields and adjacent to small woodlots. The Org. Farm (39.640873 N, -79.939141 E) site was located at an organic research farm in Morgantown, WV. This site was an unmowed hillside located near a market garden and which had been used at various times in an ongoing sheep grazing experiment. The Org. Farm site was also adjacent to a small woodlot. The Philippi (39.147658 N, -80.104626 E) site was located in a mowed field located in Elk City, an unincorporated town near Philippi, WV. This field was located near a small residential area comprising two homes and other small buildings, and adjacent to a small stream and woodlot. Study Design

A regular grid of at least 100 collection points was used at each site. Each point consisted of a pair of pan traps, one fluorescent blue and one fluorescent yellow, placed $10 \mathrm{~m}$ from the next nearest trap. Five points were selected at random from within the grid, and at these points additional pairs of traps were placed at $2 \mathrm{~m}$ and $4 \mathrm{~m}$ in each cardinal direction (i.e., geostatistical cross) (Fig. 1). These additional traps were used to detect spatial autocorrelation at smaller distances. This grid spacing was chosen based on preliminary results which failed to create useful models of spatial autocorrelation at smaller scales. Therefore, the total number of sample points per site ranged from 140-149.

Each pan trap was filled half way with soapy water and left to collect over a 5-day period. Traps were checked every other day for fluid levels, and refilled as needed. At the end of the 5day period, the number of bees in each pan trap was recorded, and all bees were collected into $70 \% \mathrm{EtOH}$. Bees were then taken back to the lab where they were identified to genus. Analysis 
Data at each site was found to be non-normal, but transformations did not yield normal data as determined by the Shapiro-Wilk normality test. All data was detrended in R version 3.3.1 before being analyzed. Data was analyzed separately for blue pan traps, yellow pan traps, and blue and yellow pan traps combined. Geostatistical analysis was done using GS+ version 9, and model selection was based on the residual sum of square (RSS), $\mathrm{R}^{2}$, and visual fitting. The range value for the model was used to determine the distance in which spatial autocorrelation occurred between points.

To determine if bee capture was biased based on bee genera, we performed chi-square analysis using $\mathrm{R}$ version 3.3.1 on count data for each site, assuming an even distribution of bee genera would be caught in pan traps. Additionally, a chi-square analysis was performed on data combined across all sites.

\section{Results}

In total, 848 bees were collected at the four sites, representing 16 genera (Table 1). The most common genus was Lasioglossum $(\mathrm{n}=632)$ followed by Augochlorella $(\mathrm{n}=73)$, and only a single individual was collected from Calliopsis, Colletes, and Triepeolus (Table 1). Chi-square results of the number of individuals of each genera caught were significant for Ag. Farm W, Ag. Farm E, Philippi, and combined data, but were not significant at Org. Farm (Table 2).

The largest distance at which spatial dependency was found between points was $17.3 \mathrm{~m}$ in blue traps at Ag. Farm W. The largest distance of spatial dependency for yellow traps was $11.3 \mathrm{~m}$, and for combined (yellow + blue) traps was $14.0 \mathrm{~m}$ (Table 3). Data were considered sparse if less than $25 \%$ of traps contained bees at the end of the 5-day collecting period. The data which fell into this category were Ag. Farm E yellow traps, Org. Farm yellow traps and blue 
traps, and Philippi yellow traps. Model parameters for this data is given, but should be interpreted cautiously.

\section{Discussion}

We found the maximum distance of spatial dependency between paired blue + yellow pan traps to be $14.0 \mathrm{~m}$, though the maximum distance of spatial dependency was longer in blue traps (17.3 m). In contrast, Droege et al. (2010) found a distance of $5 \mathrm{~m}$ was a suitable separation distance to obtain maximum capture rate in pan traps. Because geostatistical methods used in this study account for both distance and direction, and begin from the assumption that samples are spatially dependent; our results more accurately represent spatial dependence in pan trap samples when compared to results from Droege et al. (2010). For this reason we suggest using a conservative inter-bowl distance of $18 \mathrm{~m}$ when independent samples are desirable when sampling over 5 day periods in the fall. This may be the case when traditional statistical methods are to be used in analyzing pan trap data. This large distance does pose some practical problems for melittological research. First, it may make locating pan traps along a transect in the field more difficult for researchers. Second, fewer pan traps may be used within a sampling domain as the distance between the traps increases, which may be undesirable at some spatial scales.

Previous studies have indicated bias in pan trap samples (Westphal et al. 2008, Wilson et al. 2008). The results of this study indicated similar biases with smaller bees being caught in greater abundance than larger bees. Chi-square results in our study indicated that bee genera distributions were significantly different from the expected hypothesis that all bee genera would be encountered evenly at Ag. Farm W, Ag. Farm E, and Philippi, but we failed to reject the null hypothesis at Org. Farm. This is important for two reasons. First, because Org. Farm was the only site where pesticide use was not permitted, there is some indication that pesticide use may 
affect bee community structure. Second, Org. Farm was also the only site where the field was not mowed, leading to the possibility that mowed lands favor some bee genera more than others. The Org. Farm was also the only site where Lasioglossum was not the most abundant genus collected in pan traps. Instead, Agapostemon was the most abundant genus at Org. Farm, followed by Apis and Lasioglossum.

The results of this study suggest that pan traps should be placed at least $18 \mathrm{~m}$ apart when it is desirable for collected samples to be truly independent. A separation distance of $5 \mathrm{~m}$ suggested by Droege et al. (2010) may still be used when the scale of the study area is not suited to such a large separation distance, or when maximizing bee capture is more important than getting spatially independent results. Additionally, pan traps bias may be in part a result of the environments in which they are placed, as our Org. Farm results indicated that undisturbed, pesticide-free habitats may support a more even distribution of bee genera than disturbed habitats. Further information is needed in this area, as this study was not explicitly designed to answer that question. Future work on pan trap usage should be directed toward understanding how well pan trap capture reflects the reality of bee diversity.

\section{Acknowledgements}

The authors would like to thank Zane Whitesell for assisting with preliminary stages of this project. We would also like to thank Jacob Goldner, John Moredock, and Kayla McKinney for their assistance in the field.

\section{References Cited}

Bartholomew, C. S. and D. Prowell. 2005. Pan compared to malaise trapping for bees (Hymenoptera: Apoidea) in a longleaf pine savanna. J. Kans. Entomol. Soc. 78: 390-392. 
Brosi, B. J., G. C. Daily, P. R. Ehrlich. 2007. Bee community shifts with landscape context in a tropical countryside. Ecol. Appl. 17: 418-430.

Cane, J. H., R. L. Minckley, and L. J. Kervin. 2000. Sampling bees (Hymenoptera: Apiformes) for pollinator community studies: pitfalls of pan-trapping. J. Kans. Entomol. Soc. 73: 225-231.

Droege, S., V. J. Tepedino, G. LeBuhn, W. Link, R. L. Minckley, Q. Chen, and C. Conrad. 2010. Spatial patterns of bee captures in North American bowl trapping surveys. Ins. Cons. Divers. 3: 15-23.

Grundel, R., K. J. Frohnapple, R. P. Jean, and N. B. Pavlovic. 2011. Effectiveness of bowl trapping and netting for inventory of a bee community. Environ. Entomol. 40: 374-380.

Isaaks, E. H., and R. M. Srivastava. 1989. An introduction to applied geostatistics. Oxford University Press. New York.

Kalhorn, K., E. M. Barrows, and W. E. LaBerge. 2003. Bee (Hymenoptera: Apoidea: Apiformes) diversity in an Appalachian shale barrens. J. Kans. Entomol. Soc. 76: 455-468.

Kirk, W. D. J. 1984. Ecologically selective colored traps. Ecol. Entomol. 9: 35-41.

Krajewski, S. A., and B. L. Gibbs. 2001. Understanding contouring: a practical guide to spatial estimation using a computer and semivariogram interpretation. Gibbs Associates, Boulder, CO.

Leong, J. M., and R. W. Thorp. 1999. Colour-coded sampling: the pan trap color preferences of oligolectic and non-oligolectic bees associated with a vernal pool plant. Ecol. Entomol. 24: 329-335.

Moeller, D. A. 2006. Geographic structure of pollinator communities, reproductive assurance, and the evolution of self-pollination. Ecology 87: 1510-1522. 
Park, Y.-L., and J. J. Tollefson. 2006. Spatial distributions of corn rootworm (Coleoptera: Chrysomelidae) eggs and larvae: implications for sampling. J. Kans. Entomol. Soc. 79(2): 129-135.

Roulston, T. H., S. A. Smith, and A. L. Brewster. 2007. A comparison of pan trap and intensive net sampling techniques for documenting a bee (Hymenoptera: Apiformes) fauna. J. Kans. Entomol. Soc. 80: 179-181.

Stephen, W. P. and S. Rao. 2005. Unscented color traps for non-Apis bees (Hymenoptera: Apiformes). J. Kans. Entomol. Soc. 78: 373-380.

Toler, T. R. E. W. Evans, and V. J. Tepedino. 2005. Pan trapping for bees (Hymenoptera: Apiformes) in Utah's west desert: the importance of color diversity. Pan-Pac. Entomol. 81: 103-113.

Vega, F. E., P. Barbosa, and A. P. Panduro. 1990. An adjustable water-pan trap for simultaneous sampling of insects at different heights. Fla. Entomol. 73: 656-660.

Westphal, C., R. Bommarco, G. Carré, E. Lamborn, N. Morrison, T. Petanidou, S. G. Potts, S. P. M. Roberts, H. Szentgyörgyi, T. Tscheulin, B. E. Vaissière, M. Woyciechowski, J. C. Niesmeijer, W. E. Kunin, J. Settele, and I. Steffan-Dewenter. 2008. Measuring bee diversity in different European habitats and biogeographical regions. Ecol. Monogr. 78: 653-671.

Wilson, J. S. T. Griswold, and O. J. Messinger. 2008. Sampling bee communities (Hymenoptera: Apiformes) in a desert landscape: are pan traps sufficient. J. Kans. Entomol. Soc. 81: 288-300. 
Table 1. Semivariogram model parameters* for four study sites.

\begin{tabular}{lllllllll}
\hline Site & $\begin{array}{l}\text { Trap } \\
\text { color }\end{array}$ & Model & Nugget & Sill & Range & RSS & R2 & Prop \\
\hline Ag. Farm W & Combined & Gaussian & 5.10 & 11.97 & 14.03 & 10.80 & 0.81 & 0.57 \\
Ag. Farm W & Yellow & Spherical & 0.26 & 3.18 & 11.30 & 0.91 & 0.79 & 0.92 \\
Ag. Farm W & Blue & Gaussian & 2.82 & 5.79 & 17.32 & 2.93 & 0.78 & 0.51 \\
Ag. Farm E & Combined & Linear & 0.54 & - & - & 0.03 & 0.31 & 0.16 \\
Ag. Farm E & Yellow & Linear & 0.21 & - & - & 0.01 & 0.28 & 0.17 \\
Ag. Farm E & Blue & Linear & 0.30 & - & - & 0.01 & 0.09 & 0.10 \\
Org. Farm & Combined & Spherical & 0.00 & 0.36 & 12.20 & 0.04 & 0.60 & 1.00 \\
Org. Farm & Yellow & Gaussian & 0.01 & 0.06 & 5.02 & 0.00 & 0.14 & 0.91 \\
Org. Farm & Blue & Spherical & 0.00 & 0.30 & 12.60 & 0.02 & 0.65 & 1.00 \\
Philippi & Combined & Spherical & 0.18 & 0.64 & 11.10 & 0.02 & 0.72 & 0.71 \\
Philippi & Yellow & Linear & 0.14 & - & - & 0.01 & 0.66 & 0.39 \\
Philippi & Blue & Gaussian & 0.06 & 0.51 & 6.06 & 0.01 & 0.70 & 0.88 \\
\hline
\end{tabular}

*The nugget is the y-intercept of the model, the sill is the model asymptote, the range is the separation distance at which the model reaches the asymptote, and the prop. is a statistic providing a measure of the proportion of sample variance explained by spatially structured variance. 
Table 2. Bee genera found at each of the four study sites.

\begin{tabular}{lccccc}
\hline Genera [16] & Ag. Farm W & Ag. Farm E & Org. Farm & Philippi & Total \\
\hline Agapostemon & 26 & 9 & 9 & 3 & 47 \\
Andrena & 0 & 3 & 2 & 2 & 7 \\
Apis & 4 & 3 & 7 & 10 & 24 \\
Augochlora & 0 & 1 & 0 & 10 & 11 \\
Augochlorella & 57 & 7 & 5 & 4 & 73 \\
Bombus & 6 & 1 & 1 & 1 & 9 \\
Calliopsis & 0 & 1 & 0 & 0 & 1 \\
Ceratina & 0 & 0 & 6 & 1 & 7 \\
Colletes & 1 & 0 & 0 & 0 & 1 \\
Halictus & 3 & 10 & 1 & 1 & 15 \\
Hylaeus & 0 & 8 & 0 & 0 & 8 \\
Lasioglossum & 525 & 39 & 7 & 61 & 632 \\
Megachile & 0 & 0 & 4 & 0 & 4 \\
Mellisodes & 0 & 2 & 0 & 3 & 5 \\
Sphecodes & 3 & 0 & 0 & 0 & 3 \\
Triepeolus & 0 & 0 & 0 & 1 & 1 \\
\hline Total & 625 & 84 & 42 & 97 & $\mathbf{8 4 8}$
\end{tabular}


Table 3. Results of chi-square test using the hypothesis that all bee genera would be found in equal numbers at the four sites.

\begin{tabular}{llll}
\hline & $\chi^{\mathbf{2}}$ & D.F. & P \\
\hline Ag. Farm W & 2954.149 & 7 & $<0.0001$ \\
Ag. Farm E & 156.951 & 10 & $<0.0001$ \\
Org. Farm & 14.143 & 8 & 0.0781 \\
Philippi & 352.409 & 10 & $<0.0001$ \\
Combined & 6853.509 & 15 & $<0.0001$
\end{tabular}




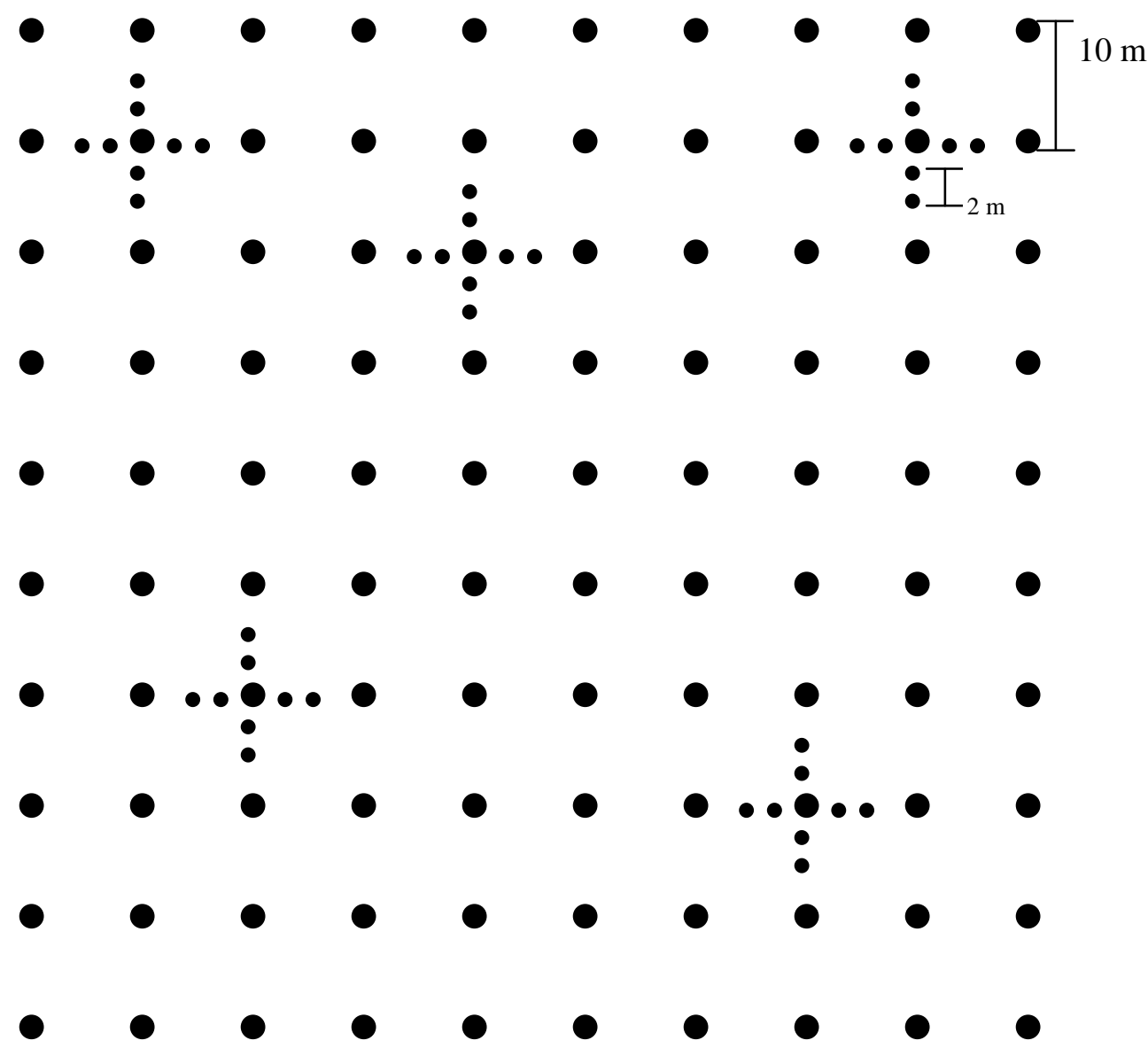

Figure 1. Example of experimental plot. Large points indicate the location of a pair of pan traps; one painted fluorescent yellow and the other fluorescent blue. Small points represent pairs of pan traps placed at smaller distance intervals to detect small scale spatial autocorrelation. 


\title{
CHAPTER 4: THERMAL BIOLOGY OF Osmia cornifrons (HYMENOPTERA: MEGACHILIDAE) EGG AND LARVAE
}

\begin{abstract}
Temperature plays an important role in the growth and development of insect, and the temperature at which an insect develops can affect both behavior and reproduction in adult insects. This study was conducted to determine the effects of temperature on development and survival in egg and larval stages of Osmia cornifrons Radozskowski (Hymenoptera: Megachilidae) over a broad range of temperatures. Osmia cornifrons individuals were placed in environmental chambers at $5,13,21,29,37$, and $45^{\circ} \mathrm{C}$ and their development was tracked daily. Developmental data was modelled using the Briére function and simulated by incorporating the developed model and Weibull function. Models were validated with developmental data from randomly selected bees at sites in Morgantown $(n=28)$ and Kearneysville $(n=78)$, West Virginia. Osmia cornifrons eggs survived at 13,21 , and $29^{\circ} \mathrm{C}$, but were unable to survive at other temperatures. Larvae survived at 21 and $29^{\circ} \mathrm{C}$, with 88.5 and $45.5 \%$ survival, respectively. The thermal window for egg development was $17.99^{\circ} \mathrm{C}$, and for larva was $12.58^{\circ} \mathrm{C}$. Nearly all validation data fell within the $95 \%$ prediction interval, indicating the models accurately represented the thermal biology of Osmia cornifrons. The narrow thermal window found in this study for $O$. cornifrons larval development indicates that $O$. cornifrons is sensitive to temperature change during this life history stage. Implications for propagation and maintenance of $O$. cornifrons populations for pollination by considering its thermal biology are discussed in this article.
\end{abstract}

Keywords: ontogeny; mason bee; Japanese hornfaced bee; thermal window, temperaturedependent development 


\section{Introduction}

Temperature plays a critical role in insect growth and development. It is well documented that temperature affects the rate at which an insect develops from immature life stages to adult (Campbell, Frazer, Gilbert, Gutierrez, \& Mackauer, 1974; Logan, Wollkind, Hoyt, \& Tanigoshi, 1976; Sharpe \& DeMichele 1977; Schoolfield, Sharpe, \& Magnuson, 1981; Wagner, Wu, Sharpe, Schoolfield, \& Coulson, 1984; Lactin, Holliday, Johnson, \& Craigen, 1995; Briére, Pracros, LeRoux, \& Pierre, 1999; Ikemoto, 2005-2008; Shi, Ge, Sun, \& Chen, 2011). Additionally, the temperature at which an insect develops may affect a number of other attributes of adult insects. For example, Apis mellifera (Hymenoptera: Apidae) larvae reared at various temperatures differed in their neurological development (Groh, Tautz, \& Rossler, 2004) and their behavior as adults (Tautz, Maier, Groh, Rossler, \& Brockmann, 2003). Immature Megachile rotundata (Hymenoptera: Megachilidae) developed at different temperatures had differences in their immunity to pathogens (Xu \& James, 2012). Eldana saccharina (Lepidoptera: Pyralidae) adults were found to have differences in water balance physiology depending on the temperature at which larvae developed (Kleynhans, Conlong, \& Terblanche, 2014). Yocum, Kemp, Bosch, and Knoblett (2006) showed that $M$. rotundata reared at different temperatures showed differences in gene expression in diapausing adults. Adult males of the agricultural pest Callosobruchus maculatus (Coleoptera: Chrysomelidae) were shown to have different sized testes and sperm when reared at different temperatures (Vasudeva, Deeming, \& Eady, 2014). These examples highlight the importance of temperature not only on the rate of insect development, but also on the survival and fitness of adult insects.

The nature of the relationship between physiological time and temperature in insects is well understood. Development from immaturity to maturity is bounded by an upper 
developmental threshold (UDT) and lower developmental threshold (LDT), and the temperature between the temperature at maximum growth $\left(T_{\text {Max }}\right)$ and LDT is known as the thermal window (van der Have, 2002; Dixon et al., 2009). The thermal window of most insects in the temperate region has a range of $20^{\circ} \mathrm{C}$ (e.g. if the LDT is $14^{\circ} \mathrm{C}$, the $T_{\text {Max }}$ may be expected to be $34^{\circ} \mathrm{C}$ ), though the range of the thermal window is narrower in tropical insects (Dixon et al., 2009). Development between UDT and LDT is generally a nonlinear function where the apex of the curve is the temperature at which the insect develops at the fastest rate. This temperature is called the optimum or maximum temperature (Logan et al., 1976; Lactin et al., 1995; Briére et al., 1999; Roy, Brodeur, \& Cloutier, 2002; van der Have, 2002; Ikemoto, 2005; Dixon et al., 2009; Shi et al., 2011), though insects may develop at higher temperatures with a reduced survivorship and developmental rate. These parameters of the developmental window are important life history features which have been adapted to suit the thermal requirements necessary for the geographic region and seasonal temperature regimes to which different insect species are exposed (Pitts-Singer, Cane, \& Trostle, 2014; Tauber, Tauber, \& Nechols, 1987; Stacey \& Fellowes 2002; Shi, Wang, Ayres, Ge, Zhong, \& Li, 2012; Wang, Shi, Chen, \& Xue, 2013).

Osmia cornifrons (Hymenoptera: Megachilidae) is a univoltine mason bee native to Japan, Korea, China, and eastern Russia. O. cornifrons was introduced into the northeastern United States in 1977 to be used as an easily managed pollinator for apple production (Batra, 1978). O. cornifrons pollination activity occurs roughly in tandem with apple bloom in the spring, when temperature is $>14^{\circ} \mathrm{C}$ (McKinney \& Park, 2012a). Larvae develop throughout the warmest parts of the summer and become adults by early September, staying in their cocoons for an overwintering period before eclosing the following spring. While Maeta, Nakanishi, Fuji, and 
Kitamura (2006) investigated temperature-dependent development of $O$. cornifrons in Japan, their study utilized a narrow range of temperatures (i.e. four temperatures between $18-30^{\circ} \mathrm{C}$ ) and thus could not determine UDT, LDT, and thermal window to fully understand its thermal biology. Here, we report results from our laboratory and field studies to investigate the effect of temperature on $O$. cornifrons development over a wide range of temperatures by using modelingvalidation-simulation approaches (Ahn et al. 2014).

The objectives of this study were to determine for $O$. cornifrons (1) the thermal window for development from egg to prepupa, (2) the maximum temperature $\left(t_{d \max }\right)$ for development, and (3) survivorship of egg and larvae at six temperatures: $5,13,21,29,37$, and $45^{\circ} \mathrm{C}$. This information will be useful for propagation and maintenance of $O$. cornifrons populations for pollination and for determining the potential for spatial and temporal pollinator/host asynchrony under various climate change scenarios.

\section{Materials and Methods}

\section{Study Insects}

Osmia cornifrons used in the experimental portion of this study were gathered from a population managed at the West Virginia University Organic Farm in Morgantown, West Virginia (39.644996 N, $79.937348 \mathrm{~W})$. Bees were collected at the organic farm to ensure that no pesticides possibly influencing mortality were introduced into the nest. Bees were managed in wooden observation nest blocks (McKinney \& Park, 2012a, 2012b), and each morning cells containing eggs which had not been there the previous morning were marked and brought into the lab. A total of 24-27 eggs were placed in an environmental chamber (Percival Scientific, 
Perry, IA; Fisher Scientific, Dubuque, IA) maintained at one of six temperatures: 5, 13, 21, 29, 37 , and $45^{\circ} \mathrm{C}$.

\section{Effects of temperature on O. cornifrons development}

Use of observation nest blocks permitted the observation of $O$. cornifrons brood development inside the nest without disturbance. Every day, each bee was checked and data on development and mortality were taken. Eggs were noted as being hatched when noticeable movement began to occur within a nest cell. Larval development was recorded through the initiation of the cocoon. Data was not collected beyond the formation of the cocoon, as to determine development beyond that point cocoons would need to be cut open, which may have adverse effects on bee development. Developmental periods of different life stages of O. cornifrons were compared by using PROC GLM in SAS (SAS Institute 2002) and Tukey's studentized range test (HSD) for mean separation at $5 \%$ error rate.

\section{Modeling temperature-dependent development of $O$. cornifrons}

We used the function proposed by Briére et al. (1999) which uses three parameters in modeling because the model better explained the temperature-dependent development of $O$. cornifrons than other models developed by Lactin et al. (1995), Logan et al. (1976), and Wagner et al. (1984). The model describes the relationship between the developmental rate of an insect and temperature, expressed as:

$$
r(T)=a\left(T-T_{L}\right) \sqrt{\left(T_{M}-T\right)}
$$

where $r(T)$ is developmental rate at each temperature, $T_{L}$ is the lower developmental threshold and $T_{M}$ is the upper developmental threshold. 
The thermal window was defined as the range in temperature between the minimum and maximum rate of development for $O$. cornifrons (Dixon et al., 2009). The temperature at the maximum developmental rate for $O$. cornifrons was calculated as follows:

$$
t_{d \max }=\frac{2 m T_{M}+(m+1) T_{L} \sqrt{4 m^{2} T_{L}^{2}+(m+1)^{2} T_{L}^{2}-4 m^{2} T_{M} T_{L}}}{4 m+2}
$$

where the definition of the parameters is derived from Briére's equation and $m$ is a constant.

The relationships between the cumulative distribution of each life stage and the normalized developmental times were fit with the two-parameter Weibull distribution function (Weibull, 1951; Wagner et al., 1984), described as:

$$
F(x)=1-\exp \left(-(x / \alpha)^{\beta}\right)
$$

where $F(x)$ is the cumulative frequency at normalized time $x$ (developmental time/mean developmental time), $\alpha$ is the scale parameter, and $\beta$ is the parameter of curve shape.

\section{Validation of models for temperature-dependent development of $O$. cornifrons}

To validate the models developed above, a field study was conducted in two fields located in Morgantown (39.644996 N, 79.937348 W) and Kearneysville (39.395519 N, 77.894104 W), West Virginia, where $O$. cornifrons colonies have been maintained the past five years in observation nests blocks. A total of 28 and 78 bee cells from the Morgantown and Kearneysville fields, respectively, were randomly selected, and the development of $O$. cornifrons from egg to prepupa was observed and recorded. Temperature in the nest of $O$. cornifrons from Morgantown was recorded with a HOBO data logger $\left(\mathrm{HOBO}{ }^{\circledR}\right.$ onset ${ }^{\circledR} \mathrm{UX} 100$ Temp/RH, Cape Cod, MA) and that from Kearneysville was obtained from the data of a weather station (Vantage Pro, Davis Instruments, Hayward, CA) located directly at the sight of the $O$. cornifrons nests. Degree days needed for development from egg to prepupal stage of $O$. cornifrons was calculated based on the 
lower developmental threshold of $13^{\circ} \mathrm{C}$ because $O$. cornifrons did not develop to the prepupal stage at $13^{\circ} \mathrm{C}$. The cumulative estimated value was calculated using sigmoid function, described as:

$$
C(x)=\frac{a}{\left[1+\exp \left(-\frac{(x-b)}{c}\right)\right]}
$$

where $\mathrm{C}(x)$ is cumulative proportion of prepupa emergence, $x$ is observed degree day from constant temperature, $a, b$, and $c$ are parameters of the equation. The upper and lower prediction intervals for an individual $\mathrm{C}(x)$ were $\hat{\mathrm{C}}(x)-\mathrm{E}$ and $\hat{\mathrm{C}}(x)+\mathrm{E}$, respectively, where $\hat{\mathrm{C}}(x)$ is predicted value of $\mathrm{C}(x)$ and $\mathrm{E}$ is error of prediction. $\mathrm{E}$ is expressed as

$$
\mathrm{E}=t \alpha / 2 s_{e} \sqrt{1+\frac{1}{n}+\frac{n\left(C_{0}-C_{\text {average }}\right)^{2}}{n\left(\sum C^{2}\right)-\left(\sum C\right)^{2}}}
$$

where $n$ is sample size, $s_{e}$ is standard error of sample and $C_{0}$ is observed data. To validate the model, $95 \%$ prediction intervals were used to compare field-observed data with predicted values from the model.

\section{Simulation of temperature-dependent development of O. cornifrons}

The developmental frequency of $O$. cornifrons egg, larva, and egg to larva stage was simulated in relation to time (day) and temperature by combining the non-linear model and the Weibull function, described as:

$$
F(x, T)=1-\exp \left[-\left(\frac{x r(T)}{\alpha}\right)^{\beta}\right]
$$

where $F(x, T)$ is the frequency of termination of $O$. cornifrons egg, larva, and egg to larva stages at time $x$ and constant temperature $T, x$ is time (day), $r(T)$ is the non-linear function for developmental rate, and $\alpha$ and $\beta$ are parameters from the Weibull equation. The resultant values 
from the simulation were presented as frequency of termination of different life stage emerging at $x_{i}$, which was estimated by subtracting the frequency of the simulation result at $x_{i}$ from that of $x_{i+1}$

\section{Results}

\section{Effects of temperature on O. cornifrons development}

The development periods of $O$. cornifrons egg and larva were significantly influenced by temperature $\left(\mathrm{F}_{2,69}=1690.77, \mathrm{p}<0.0001\right.$ for egg; $\mathrm{F}_{1,30}=19.44, \mathrm{p}<0.0001$ for larva; $\mathrm{F}_{1,30}=$ 11.26, $\mathrm{p}<0.0022$ for egg to larva). $O$. cornifrons eggs developed successfully to the prepupal stage at 21 and $29^{\circ} \mathrm{C}$ (Table 1), but no eggs hatched at 5, 37 and $45^{\circ} \mathrm{C}$. Eggs survived to the larval stage but failed to develop into prepupa at $13^{\circ} \mathrm{C}$. The larval stage took significantly more time than the egg stage to complete development at $21^{\circ} \mathrm{C}\left(\mathrm{F}_{1,47}=1902.36, \mathrm{p}<0.0001\right)$ and at $29^{\circ} \mathrm{C}\left(\mathrm{F}_{1,29}=986.76, \mathrm{p}<0.0001\right)$.

\section{Modeling temperature-dependent development of $O$. cornifrons}

The relationship between the developmental rate of immature $O$. cornifrons and temperature was described with linear and Briére 1 functions for the egg stage and with only the Briére 1 function for the larval stage (Figure 1 and Table 2). The lower developmental threshold and thermal constant of eggs using linear regression were $12.73^{\circ} \mathrm{C}$ and 16.66 degree days (DD), respectively. The development data of $O$. cornifrons fit well with the Briére 1 model because the parameters of the model explained $97 \%$ and $87 \%$ of the variabilities for egg and larval stages, respectively. The upper developmental threshold for eggs was higher than that of larvae. The cumulative proportion against normalized time was explained well by the two-parameter Weibull function 
(Figure 2 and Table 3). Development from egg to larva was less variable, as indicated by the higher value for the $\beta$ parameter, than development from larva to prepupa. The temperature range for development from larva to prepupa was narrower than that for egg to larva. The thermal windows between $T_{L}$ and $T_{\text {Max }}$ of egg to larva, larva to prepupa and egg to prepupa were 17.99, 12.58 , and $12.71^{\circ} \mathrm{C}$, respectively.

\section{Validation of models for temperature-dependent development of O. cornifrons}

The developmental model of $O$. cornifrons from egg to prepupa was validated using the data collected from two fields. The data from both locations were within the upper and lower $95 \%$ prediction interval $\left(a=0.8794, b=236.2044, c=20.7655, \mathrm{r}^{2}=0.95, \mathrm{~F}_{2,15}=144.96, \mathrm{p}<0.0001\right)$ (Figure 3), indicating that the developed model was validated.

\section{Simulation of temperature-dependent development of O. cornifrons}

The output of the simulation for temperature-dependent development showed O. cornifrons development of egg-larva, larva-prepupa, and egg-prepupa over the entire range of temperatures at which O. cornifrons can survive and develop (Figure 4). Results of the simulation indicated that different life stages of $O$. cornifrons would require different temperature and degree day accumulation to finish their development. The highest frequencies found from the simulation were $98.2 \%$ in two days at $26^{\circ} \mathrm{C}$ and $14.7 \%$ in 25 days at $30^{\circ} \mathrm{C}$ for egg and larval development, respectively. 


\section{Discussion}

Previous research has elucidated the role temperature plays in $O$. cornifrons spring emergence (White, Son, \& Park, 2009) and in O. cornifrons nesting activity (McKinney \& Park, 2012a). Here we have shown that the range of the thermal window for $O$. cornifrons development from egg to prepupa is $12.71^{\circ} \mathrm{C}$. Dixon et al. (2009) showed the average range of the thermal window of 74 non-dormant populations of 66 species of insects was $19.8^{\circ} \mathrm{C}$ with $95 \%$ confidence interval $\left(19.1-20.5^{\circ} \mathrm{C}\right)$. The thermal windows found in our study were much narrower than the average value reported by Dixon et al. (2009), indicating that $O$. cornifrons is more sensitive to changes in temperature than the 66 insects measured in that study. Specifically, O. cornifrons larval stage (thermal window $=12.58^{\circ} \mathrm{C}$ ) is more sensitive to temperature change than the egg stage (thermal window $=17.99^{\circ} \mathrm{C}$ ). The results indicate that thermal requirements for egg and larval development are different, suggesting that $O$. cornifrons undergoes physiological changes to account for seasonal shifts in temperature from spring into summer.

A similar study with $O$. cornifrons was done in Japan. Maeta et al. (2006) studied the development and diapause temperature requirements for $O$. cornifrons for manipulating seasonal emergence in Japan. In their study $O$. cornifrons development was tracked at four temperatures $\left(18,22,26\right.$, and $\left.30^{\circ} \mathrm{C}\right)$. Our study did not share any temperature with Maeta et al. (2006) for comparison between studies, but 21 and $29^{\circ} \mathrm{C}$ used in our study were close enough to 22 and $30^{\circ} \mathrm{C}$ used in their study for comparative purposes. They found egg development times of $4.5 \pm$

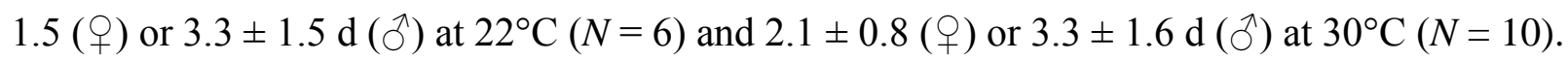
We found eggs developed in $2.31 \pm 0.094 \mathrm{~d}$ at $21^{\circ} \mathrm{C}(N=26)$ and $1.00 \pm 0.000 \mathrm{~d}$ at $29^{\circ} \mathrm{C}(N=$ 22). Differences in egg development are likely influenced by several factors. First, our experimental design did not separate out male vs. female development whereas Maeta et al. 
(2006) did. Second, there was higher variation and a lower number of observations in Maeta et al. (2006), leading to less confidence that their results are truly representative of $O$. cornifrons populations. Third, the way "hatched" eggs are defined in our study and in Maeta (2006) may not be the same, though Maeta (2006) does not clearly define hatching in that study. Finally, there may be regional differences in $O$. cornifrons populations in the two studies.

Osmia cornifrons larval development was found to be faster in the study by Maeta et al. (2006) when compared to ours. Female O. cornifrons developed from first instar to the cocooning stage in $11.9 \pm 1.9$ at $22^{\circ} \mathrm{C}(N=40)$ and $8.7 \pm 1.2 \mathrm{~d}$ at $30^{\circ} \mathrm{C}(N=45)$, while male $O$. cornifrons larvae developed to cocooning in $11.3 \pm 1.5$ at $22^{\circ} \mathrm{C}(N=17)$ and $8.4 \pm 1.3 \mathrm{~d}$ at $30^{\circ} \mathrm{C}$ $(N=30)$. Larvae from our study developed to prepupae in $26.74 \pm 0.600$ at $21^{\circ} \mathrm{C}(N=26)$ and $32.17 \pm 1.721 \mathrm{~d}$ at $29^{\circ} \mathrm{C}(N=22)$. The large differences in observed development time at similar temperatures between the two studies may be caused by genotypic differences in $O$. cornifrons populations in Japan and the United States.

Bosch and Kemp (2000) studied the effects of temperature on the development of $O$. lignaria, a managed pollinator closely related to $O$. cornifrons and the most popularly used pollinator in the United States. In their study, O. lignaria successfully developed to adulthood at temperatures from 18 to $29^{\circ} \mathrm{C}$ which were the minimum and maximum temperatures used in the study. Our study and that of Bosch \& Kemp (2000) used different temperatures, but did share $29^{\circ} \mathrm{C}$ as a common temperature for comparison between $O$. lignaria and $O$. cornifrons. At $29^{\circ} \mathrm{C}$ O. cornifrons eggs took a single day to hatch, where $O$. lignaria took $5.7(\circlearrowright)$ or 7.1 (†) days. This indicates $O$. cornifrons eggs develop faster than those of $O$. lignaria. Conversely, $O$. lignaria developed from first instar to cocooning $(11.7 \mathrm{~d} \widehat{\delta}$ and $12.4 \mathrm{~d} \uparrow)$ faster than $O$. cornifrons $(32.78 \mathrm{~d})$ at $29^{\circ} \mathrm{C}$. When these times are combined it is clear that $O$. lignaria develops 
from egg to cocooning faster than $O$. cornifrons, though differences in observed development times for each stage may be due to a number of factors including differences in the way each developmental stage was defined in the two studies.

Climate change has been shown to have effects on population dynamics of insects including spatial and temporal asynchrony of interacting populations (see Field, Lobell, Peters, \& Chiariello, 2007; Pautasso et al., 2010 for reviews). The Coupled Model Intercomparison Project Phase 5 (CMIP5; Taylor, Stouffer, \& Meehl, 2012) predicted that future climate changes would have severe impacts on various ecosystems. Among various events caused by climate change, temperature increase could be the most influential driver of ecosystem function (Schweiger, Settele, Kudrna, Klotz, \& Kühnet, 2008). While some social bees (e.g. honey bees) can control the temperature of their hives by fanning their wings, solitary bees such as $O$. cornifrons do not have the ability to manipulate temperature within their nests. The results of our study showed that $O$. cornifrons was unable to complete larval development under $13^{\circ} \mathrm{C}$ and no eggs hatched at $>37^{\circ} \mathrm{C}$, indicating that development of $O$. cornifrons is sensitive to temperature changes, especially in the case of larvae. White et al. (2009) showed that $O$. cornifrons emergence is also sensitive to temperature change. Therefore, small changes in temperature could result in spatial and temporal changes in $O$. cornifrons phenology. It should be noted that this interpretation is limited by the nature of the study design. That is, results from constant temperature experiments may not reflect developmental limitations due to the fluctuating thermal conditions of the natural environment.

The narrow thermal window found for $O$. cornifrons leads to some important considerations and complications for managing bees for agricultural production and for annual population increases. Bee release must be synchronized with the onset of crop bloom for 
agricultural production, but some crops bloom during times where temperatures fall outside the ideal range for $O$. cornifrons development. For example, apple (Malus domestica L.) bud break occurs in West Virginia in late March and the trees are in bloom by the third to fourth week of April. O. cornifrons are released approximately two weeks prior to bloom, and the first eggs are laid shortly after mating commences. The average high temperature in Kearneysville, West Virginia during April is $17.5^{\circ} \mathrm{C}$ and the average low is $3.8^{\circ} \mathrm{C}$, and the LDT we found for $O$. cornifrons eggs $\left(11.4 \pm 2.18^{\circ} \mathrm{C}\right)$ falls within this temperature range. While the average high temperature in April is acceptable for O. cornifrons egg development, the average low temperature is well below the LDT. Eggs laid during this period of apple bloom may experience higher mortality due to cold nighttime temperatures than those laid later during the season. At this time it may be advisable to protect $O$. cornifrons nests from low temperature.

Because $O$. cornifrons is an introduced species in the United States, and native to several eastern Palearctic countries, future work should be directed to understanding regional differences in the thermal requirements of $O$. cornifrons. Additionally, developing models of $O$. cornifrons temperature-dependent development for male and female bees separately, and for prepupa to adult life stage, would be worthwhile for determining optimal rearing conditions. Finally, because $O$. cornifrons development is sensitive to temperature change, investigating multitrophic interactions between $O$. cornifrons, its host plants (specifically those of agricultural importance), predators, parasites, and competitors will permit a much more realistic estimate of the effects of climate change on $O$. cornifrons populations. This holds true not only for $O$. cornifrons, but for any pollinator/host relationships, and especially for specialist (i.e. monolectic) pollinators. 


\section{Acknowledgements}

We thank Vicki Kondo (West Virginia University) and Bob McConnell (grower in Independence, West Virginia) for their help with construction of bee nests for this study. The work was supported by the United States Department of Agriculture, National Institute of Food and Agriculture under grant [NIFA 2014-67004-21631].

\section{References Cited}

Ahn, J.J., Park, Y.-L., \& Jung, C. (2014). Modeling spring emergence of Osmia cornifrons Radoszkowski (Hymenoptera: Megachilidae) females in Korea. Journal of Asia-Pacific Entomology, 17, 901-905.

Batra, S. (1978). Osmia cornifrons and Pithitis smaragdula, two Asian bees introduced into the United States for crop pollination. Proceedings of the IVth International Symposium on Pollination, 1, 307-312.

Bosch, J., \& Kemp, W.P. (2000). Development and emergence of the orchard pollinator Osmia lignaria (Hymenoptera: Megachilidae). Environmental Entomology, 29, 8-13.

Briére, J.P., Pracros, P., LeRoux, A.Y., \& Pierre, J.S. (1999). A novel rate model of temperaturedependent development for arthropods. Environmental Entomology, 28, 22-29.

Campbell, A., Frazer, B.D., Gilbert, N., Gutierrez, A.P., \& Mackauer, M. (1974). Temperature requirements of some aphids and their parasites. Journal of Applied Ecology, 11, 431438.

Dixon, A.F.G., Honěk, A., Kell, P., Kotela, M.A.A., Šizling, A.L., \& Jarošik, V. (2009). Relationship between the minimum and maximum temperature thresholds for development in insects. Functional Ecology, 23, 257-264. 
Field, C.B., Lobell, D.B., Peters, H.A., \& Chiariello, N.R. (2007). Feedbacks of terrestrial ecosystems to climate change. Annual Review of Environmental Resources, 32, 1-29.

Groh, C., Tautz, J., \& Rossler, W. (2004). Synaptic organization in the adult honey bee brain is influenced by brood-temperature control during pupal development. Proceedings of the National Academy of Sciences, 101, 4268-4273.

Ikemoto, T. (2005). Intrinsic optimum temperature for development of insects and mites. Environmental Entomology, 34, 1377-1387.

Ikemoto, T. (2008). Tropical malaria does not mean hot environments. Journal of Medical Entomology, 45, 963-969.

Kleynhans, E., Conlong, D.E. \& Terblanche, J.S. (2014). Direct and indirect effects of development temperature on adult water balance traits of Eldana saccharina (Lepidoptera: Pyralidae). Journal of Insect Physiology, 68, 69-75.

Lactin, D.J., Holliday, N.J., Johnson, D.L., \& Craigen, R. (1995). Improved rate model of temperature-dependent development by arthropods. Environmental Entomology, 24, 6875.

Logan, J.A., Wollkind, D.J., Hoyt, S.C., \& Tanigoshi, L.K. (1976). An analytic model for description of temperature dependent rate phenomena in arthropods. Environmental Entomology, 5, 1133-1140.

Maeta, Y., Nakanishi, K., Fuji, K., \& Kitamura, K. (2006). Exploitation of systems to use a univoltine Japanese Mason Bee, Osmia cornifrons (Radoszkowski), throughout the year for pollination of greenhouse crops (Hymenoptera, Megachilidae). Chugoku Kontyu, 20, $1-17$. 
McKinney, M. \& Park, Y.-L. (2012a). Nesting activity and behavior of Osmia cornifrons (Hymenoptera: Megachilidae) elucidated using videography. Psyche, 2012, 1-7.

McKinney, M. \& Park, Y.-L. (2012b). Distribution of Chaetodactylus krombeini (Acari: Chaetodactylidae) within Osmia cornifrons (Hymenoptera: Megachilidae) nests: implications for population management. Experimental and Applied Acarology, 60, 153161.

Pautasso, M., Dehnen-Schmutz, K., Holdenrieder, O., Pietravalle, S., Salama, N., Jeger, M.J. Lange, E., \& Hehl-Lange, S. (2010). Plant health and global change - some implications for landscape management. Biological Reviews, 85, 729-755.

Pitts-Singer, T.L., Cane, J.H., \& Trostle, G. (2014). Progeny of Osmia lignaria from distinct regions differ in developmental phenology and survival under a common thermal regime. Journal of Insect Physiology, 67, 9-19.

Roy, M., Brodeur, J., \& Cloutier, C. (2002). Relationship between temperature and developmental rate of Stethorus punctillum (Coleoptera: Coccinellidae) and its prey Tetranychus mcdanieli (Acarina: Tetranychidae). Environmental Entomology, 31, 177187.

SAS Institute. (2002). SAS user's guide; statistics version 9.1ed. SAS Institute, Cary, NC.

Schoolfield, R.M., Sharpe, P.J.H., \& Magnuson, C.E. (1981). Non-linear regression of biological temperature-dependent rate models based on absolute reaction-rate theory. Journal of Theoretical Biology 88, 719-731.

Sharpe, P.J.H., \&DeMichele, D.W. (1977). Reaction kinetics of poikilotherm development. Journal of Theoretical Biology, 64, 649-670. 
Shi, P., Ge, F., Sun, Y., \& Chen, C. (2011). A simple model for describing the effect of temperature on insect development rate. Journal of Asia-Pacifc Entomology, 14, 15-20.

Shi, P., Wang, B., Ayres, M.P., Ge, F., Zhong, L., \& Li, B.-L. (2012). Influence of temperature on the northern distribution limits of Scirpophagaincertulas Walker (Lepidoptera: Pyralidae) in China. Journal of Thermal Biology, 37, 130-137.

Stacey, D.A., \& Fellowes, M.D.E. (2002). Temperature and the development rates of thrips: evidence for a constraint on local adaptation? European Journal of Entomology, 99, 399404.

Schweiger, O., Settele, J., Kudrna, O., Klotz, S., \& Kühnet, I. (2008). Climate change can cause spatial mismatch of trophically interacting species. Ecology, 89, 3472-3479.

Tauber, C.A., Tauber, M.J., \& Nechols, J.R. (1987). Thermal requirements for development in Chrysopaoculata: A geographically stable trait. Ecology, 68, 1479-1487.

Tautz, J., Maier, S., Groh, C., Rossler, W., \& Brockmann, A. (2003). Behavioural performance in adult honey bees is influenced by the temperature experienced during their pupal development. Proceedings of the National Academy of Sciences, 100, 7343-7347.

Taylor, K. E., R. J. Stouffer, \& Meehl, G. A. (2012). An Overview of CMIP5 and the experiment design. Bulletin of the American Meteorological Society, 93, 485-498.

van der Have, T.M. (2002). A proximate model for thermal tolerance in ectotherms. Oikos, 98, 141-155.

Vasudeva, R., Deeming, D.C., \& Eady, P.E. (2014). Developmental temperature affects the expression of ejaculatory traits and the outcome of sperm competition in Callosobruchus maculatus. Journal of Evolutionary Biology, 27, 1811-1818. 
Wagner, T.L., Wu, H.I., Sharpe, P.J.H., Schoolfield, R.M., \& Coulson, B.N. (1984). Modeling insect development rates: A literature review and application of a biophysical model. Annals of the Entomological Society America, 77, 208-225.

Wang, L., Shi, P., Chen, C., \& Xue, F. (2013). Effect of temperature on the development of Laodelphax striatellus (Homoptera: Delphacidae). Journal of Economic Entomology, $106,107-114$.

Weibull, W. (1951). A statistical distribution functions of wide applicability. Journal of Applied Mechanics, 18, 293-297.

White, J,, Son, Y., \& Park, Y.-L. (2009). Temperature-dependent emergence of Osmia cornifrons (Hymenoptera: Megachilidae) adults. Journal of Economic Entomology, 102, 2026-2032.

Xu, J., \& James, R.R. (2012). Temperature stress affects the expression of immune response genes in the alfalfa leafcutting bee (Megachile rotundata). Insect Molecular Biology, 21, 269-280.

Yocum, G.D., Kemp, W.P., Bosch, J., \& Knoblett, J.N. (2006) Thermal history influences diapause development in the solitary bee Megachile rotundata. Journal of Insect Physiology, 52, 1113-1120. 
Table 1. Mean developmental times (days) (mean \pm SEM) and survivorship (\%) of Osmia cornifrons at different constant temperatures.

\begin{tabular}{lllll}
\hline Stage & $\begin{array}{l}\text { Temperature } \\
\left({ }^{\circ} \mathrm{C}\right)\end{array}$ & $\begin{array}{l}\text { Number of } \\
\text { bees }\end{array}$ & $\begin{array}{l}\text { Mean } \pm \text { SEM } \\
\text { developmental days }\end{array}$ & Survival (\%) \\
\hline Egg to Larva & 5 & 25 & $-^{*}$ & 0 \\
& 13 & 24 & $25.63 \pm 0.586 \mathrm{a}^{* *}$ & 100 \\
& 21 & 26 & $2.31 \pm 0.094 \mathrm{~b}$ & 100 \\
& 29 & 22 & $1.00 \pm 0.000 \mathrm{c}$ & 100 \\
Larva to Prepupa & 37 & 27 & - & 0 \\
& 45 & 23 & - & 0 \\
& 5 & 25 & - & 0 \\
& 13 & 24 & - & 0 \\
Egg to Prepupa & 21 & 26 & $26.74 \pm 0.600 \mathrm{~b}$ & 88.5 \\
& 29 & 22 & $32.78 \pm 1.721 \mathrm{a}$ & 45.5 \\
& 37 & 27 & - & 0 \\
& 45 & 23 & - & 0 \\
& 5 & 25 & - & 0 \\
& 13 & 24 & - & 0 \\
& 21 & 26 & $29.00 \pm 0.649 \mathrm{~b}$ & 88.5 \\
& 29 & 22 & $33.78 \pm 1.721 \mathrm{a}$ & 45.5 \\
& 37 & 27 & - & 0 \\
& 45 & 23 & - & 0 \\
\hline
\end{tabular}

* no individual survived.

** means with the same letter in a column are not significantly different $(p<0.05$, Tukey's HSD test). 
Table 2. Parameter estimates $( \pm$ SEM) for nonlinear developmental rate model (Briére 1 model) for Osmia cornifrons.

* an empirical constant

\begin{tabular}{llll}
\hline Stage & Parameter & Estimate \pm SEM & $\mathrm{r}^{2}$ \\
\hline Egg to Larva & $\mathrm{a}^{* *}$ & $0.0006 \pm 0.00011$ & \\
& $T_{L}^{* *}$ & $11.3834 \pm 2.18140$ & \\
& $T_{M}^{* * *}$ & $37.0000 \pm 2.99 \times 10^{-8}$ & \\
Larva to Prepupa & $\mathrm{a}$ & $0.00005 \pm 0.00002$ & \\
& $T_{L}$ & $10.5410 \pm 3.56800$ & 0.92 \\
& $T_{M}$ & $30.3219 \pm 1.41120$ & \\
Egg to Prepupa & $\mathrm{a}$ & $0.00004 \pm 0.00002$ & \\
& $T_{L}$ & $10.5410 \pm 3.56860$ & \\
& $T_{M}$ & $30.4856 \pm 1.56760$ & \\
\end{tabular}


Table 3. Estimated parameters of Weibull function for Osmia cornifrons.

\begin{tabular}{llll}
\hline Stage & Parameter & Estimate \pm SEM & $\mathrm{r}^{2}$ \\
\hline Egg to Larva & $\alpha^{*}$ & $1.0054 \pm 0.0021$ & 0.99 \\
& $\beta^{* *}$ & $8.8528 \pm 0.2261$ & \\
Larva to Prepupa & $\alpha$ & $1.0114 \pm 0.0039$ & 0.97 \\
& $\beta$ & $9.8419 \pm 0.6269$ & \\
Egg to Prepupa & $\alpha$ & $1.0071 \pm 0.0046$ & \\
& $\beta$ & $10.3622 \pm 0.8670$ & \\
* scale parameter & & \\
** & &
\end{tabular}




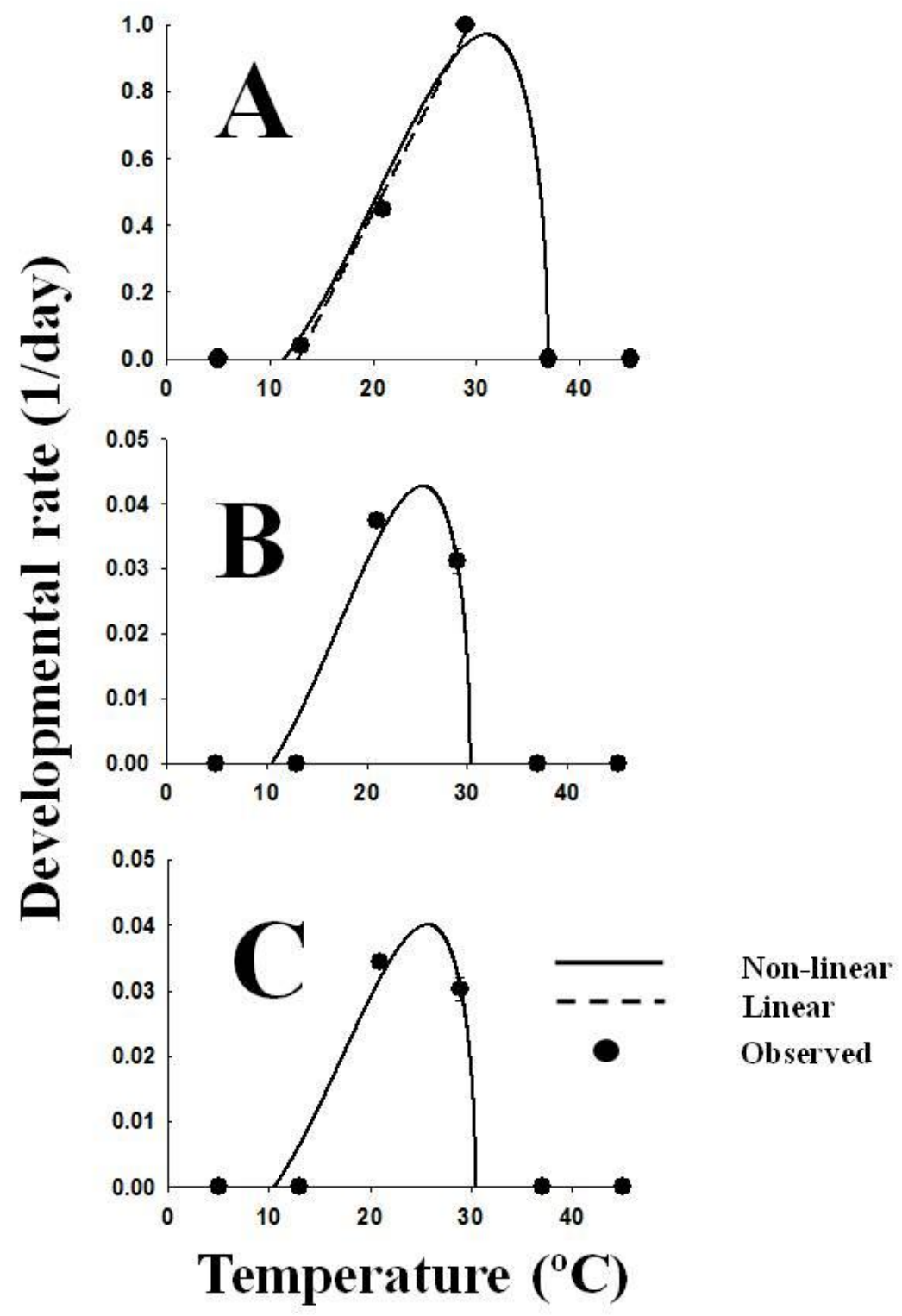

Figure 1. Relationships between temperature and developmental rates $\left(\right.$ day $\left.^{-1}\right)$ of Osmia cornifrons: A. Egg to larva, B. Larva to prepupa, C. Egg to prepupa. Linear regression of the egg stage is $r(T)=0.0600 T-0.7646$ with the lower developmental threshold of $12.73^{\circ} \mathrm{C}$ and thermal constant of 16.66 degree-days (DD). 


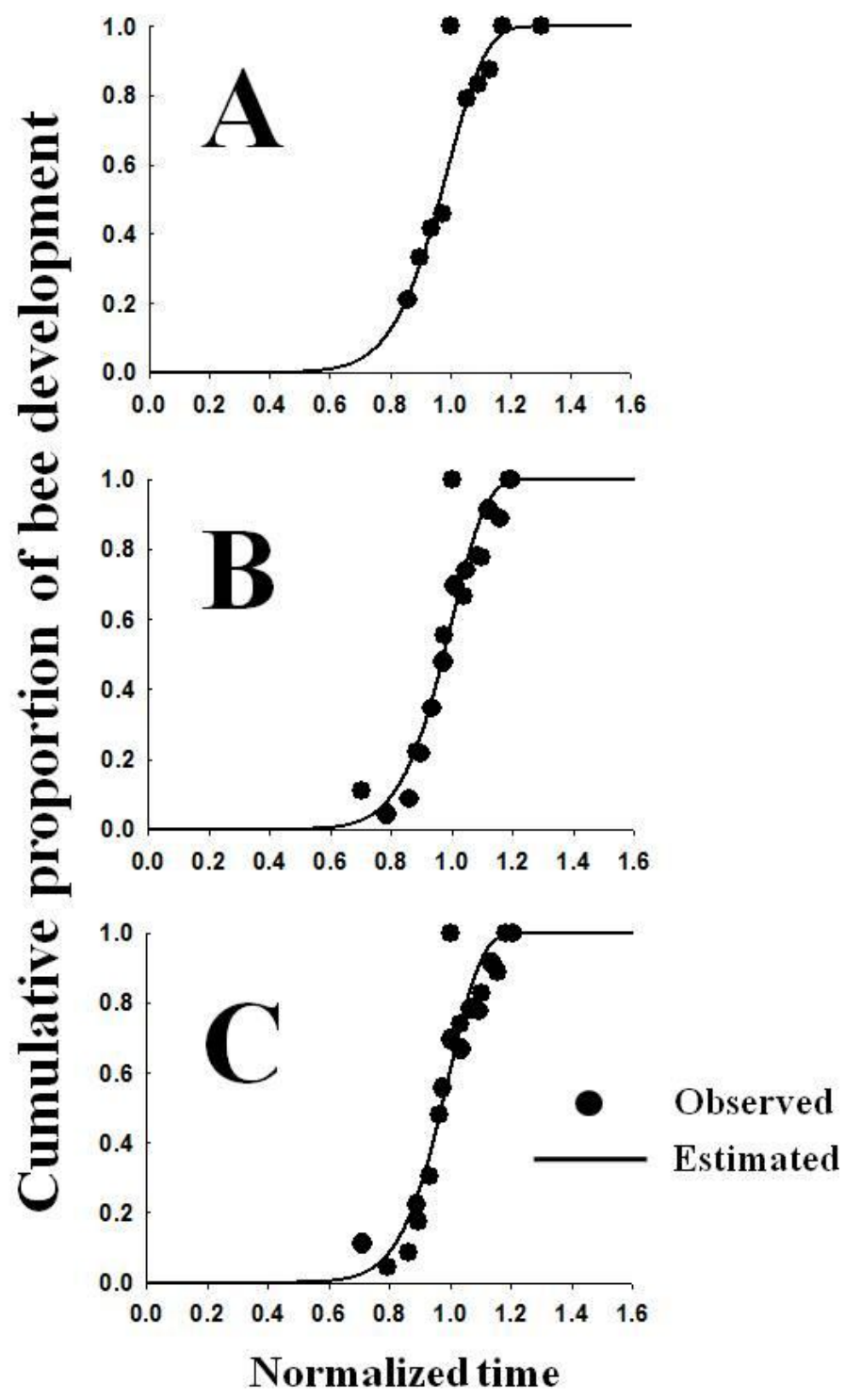

Figure 2. Observed cumulative proportions of Osmia cornifrons developed at normalized developmental time (i.e. developmental time / mean developmental time) fit with the twoparameter Weibull function: A. Egg to larva, B. Larva to prepupa, C. Egg to prepupa. 


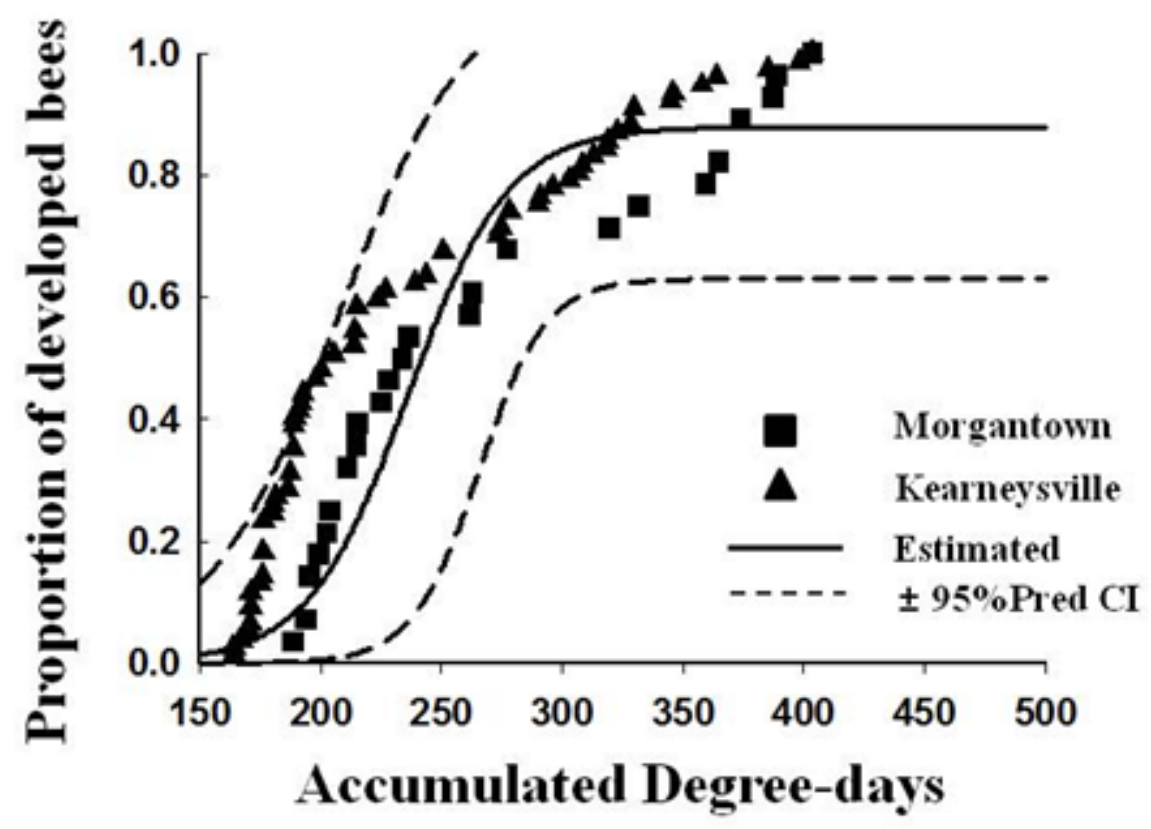

Figure 3. Validation of temperature-dependent development model for egg to prepupal stage of Osmia cornifrons by using field data collected from two locations (Morgantown and Kearneysville) in West Virginia. 


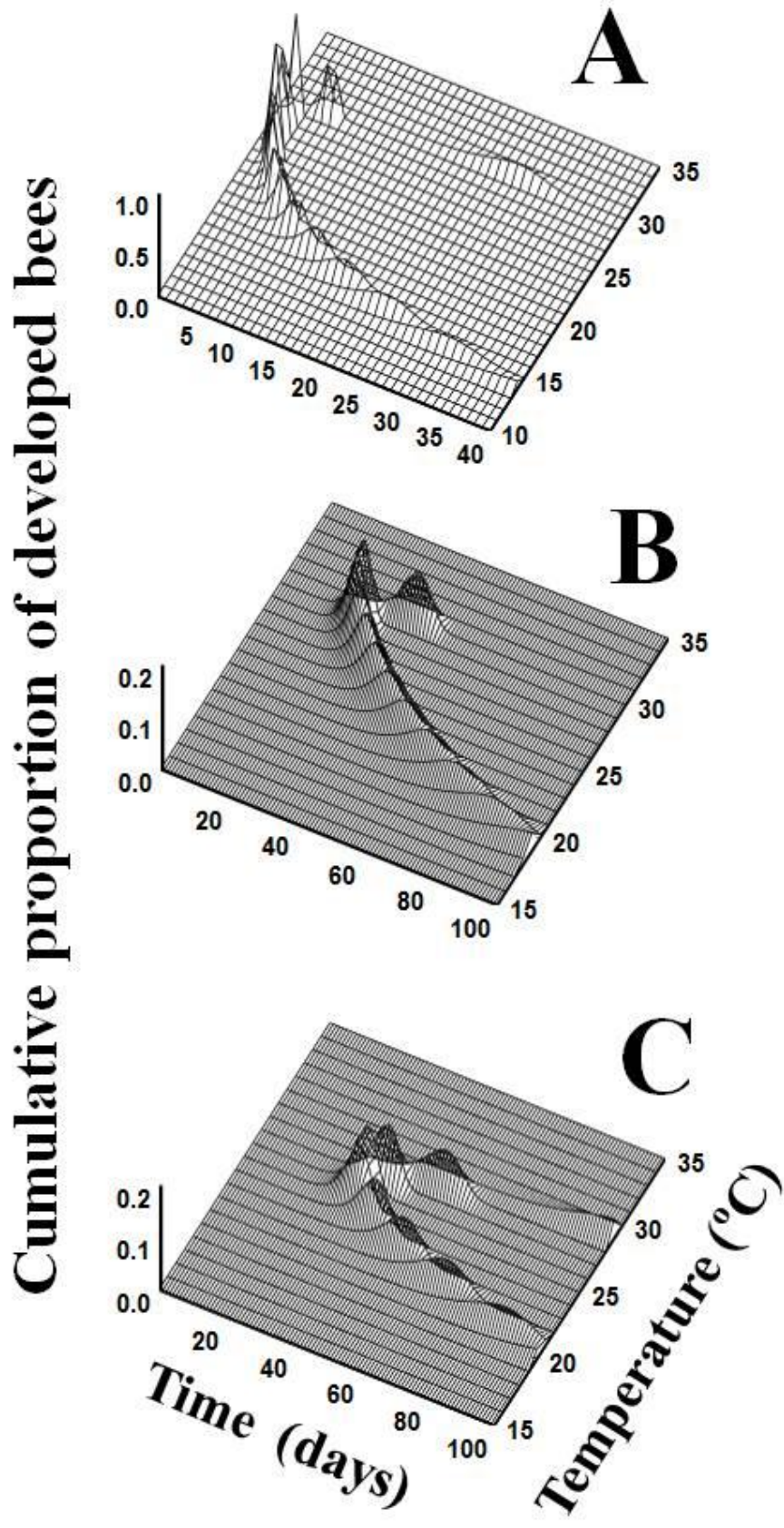

Figure 4. Simulated frequency of temperature-dependent development of Osmia cornifrons: A. Egg to larva, B. Larva to prepupa, and C. Egg to prepupa. 


\title{
CHAPTER 5: SPATIAL TRENDS OF Monodontomerus WESTWOOD (HYMENOPTERA: TORYMIDAE) IN NESTS OF Osmia cornifrons (HYMENOPTERA: MEGACHILIDAE)
}

\begin{abstract}
Monodontomerus (Hymenoptera: Torymidae) is a parasitic wasp of solitary

Megachilidae, such as Osmia (Hymenoptera: Megachilidae). Monodontomerus eggs are laid inside nest cells of the host bee and when the eggs hatch the host bee larvae is consumed by the developing wasps; this makes Monodontomerus a key pest in Osmia propagation for orchard pollination. To determine the intra-nest distribution of Monodontomerus in cardboard straws, 122 Mondontomerus infested cardboard straws were taken from a blueberry farm located in Independence, WV, and the number and position of emergence holes in each nest was recorded. To determine the distribution of Monodontomerus in bundles of nest straws, $10 \times 10$ square bundles of straws containing $O$. cornifrons were placed at the West Virginia University Organic Farm in Morgantown, West Virginia, and $12 \times 13$ bundles were placed at an apple orchard in Kearneysville, WV. Each nest in a bundle was assigned a geocoordinate and the number of Monodontomerus emergence holes was recorded. The intra-nest distribution of Monodontomerus emergence holes was found to be strongly skewed toward the outermost cells of $O$. cornifrons nests, and the spatial pattern of Monodontomerus exit holes in nest bundles showed strong anisotropy indicating Monodontomerus preferred nests on the outer perimeter of the nest bundle. The results of this study indicate that when managing Osmia in cardboard straw bundles, male Osmia bees located in nests on the outside of the bundle are highly susceptible to parasitism by Monodontomerus.
\end{abstract}

Keywords: Monodontomerus, Osmia, geostatistics, kriging, parasitism, bee nest 


\section{Introduction}

Osmia cornifrons Radoszkowski (Hymenoptera: Megachilidae) is a solitary bee managed in cardboard straws and wooden nest blocks which has been shown to be a highly effective pollinator in many fruit crop systems. In some instances $O$. cornifrons may be a more effective pollinator than Apis mellifera (Hymenoptera: Apidae) (Abel et al. 2003, Lee et al. 2008). One concern of growers utilizing $O$. cornifrons for pollination is the ability to maintain or increase population size every season. While some factors affecting $O$. cornifrons propagation are difficult or impossible to control (i.e., weather), there are a number of parasites and predators (Krunic et al. 2005, Bosch and Kemp 2001) which may be eliminated or reduced with the right management techniques. Each of these pests have specific ecological strategies which dictate the individual hosts in a population which are most likely to be negatively impacted. The linear nature of Osmia nests (and those of many other solitary bees) combined with the construction of intra-nest barriers (i.e., mud walls) creates an interaction between the life histories of Osmia and its parasites that drives predictable spatial patterns. Monodontomerus wasps are among the most commonly encountered of these pests in nests of $O$. cornifrons.

Members of Monodontomerus Westwood (Hymenoptera: Torymidae) are parasitoids of Lepidoptera, Diptera, and Hymenoptera, and may utilize the parasites of their primary hosts as secondary hosts (i.e., hyperparasitoids). For example, the primary hosts of Monodontomerus aereus Walker are members of the families Tortricidae, Lymantriidae, and Lasiocampidae, but

they have also been recorded from the secondary hosts Tachinidae, Braconidae, Ichnuemonidae, and Chalcidae. Another species, M. aeneus, parasitizes Osmia, Megachile, and Stelis, but also has been reared from Chrysididae found in nests of Megachile and Trypoxylon (see Grissel 2000, 
2007 for a comprehensive list of primary and secondary hosts). These parasitoid wasps are of economic importance both as biological control agents (Howard and Fiske 1911, McGugan and Coppel 1962, Washburn 1984, Digweed et al. 2009, Van Driesche et al 2010) and as pests (Eves 1970, Parker and Torchio 1980), and may be found in nearly all faunal regions of the world (Gahan 1941).

Previous studies which described the biology of Monodontomerus on aculeate Hymenopteran hosts include studies on M. aeneus (misidentified as M. obscurus, see Grissel 2000) (Eves 1970, Hobbs and Krunic 1971, Goodpasture 1975) and M. mandibularis (Rau 1947). Monodontomerus first locates a host through volatile cues such as acetic acid produced by bee frass (Fillela et al. 2011) and pine tree resin used in bee nest building (Macivor and Salehi 2014). Once located, Monodontomerus enters the bee nest and locates a prepupal host in a cocoon. The wasp then saws through the cocoon with its ovipositor and injects the host with paralytic fluids (Eves 1970). Once the host is paralyzed, Monodontomerus lays a number of eggs (Eves 1970 observed between 3-51 eggs laid on a single host) which will begin hatching within a couple days into hemolymph feeding first instar larvae. The number of generations per year varies by species, for example M. obscurus has 2-4 generations per year (Bosch and Kemp 2001) whereas Monodontomerus dentipes has two generation per year (Grissel 2000). Monodontomerus overwinters in nests as mature larvae and emerges with hosts the following spring. Sex ratio is most often skewed toward females (Hobbs and Krunic 1971).

The goal of this study was to determine the potential impact of Monodontomerus parasitism on $O$. cornifrons populations. To do this we first determined the distribution of male and female $O$. cornifrons in cardboard straws. We then determined the distribution of Monodontomerus emergence holes to elucidate the impact Monodontomerus had on male and 
female $O$. cornifrons. Finally, we used geostatistics to determine the spatial distribution of Monodontomerus in cardboard straw bundles. No previous studies have investigated the spatial interactions of $O$. cornifrons and Monodontomerus.

\section{Materials and Methods}

Intra-nest distribution Osmia cornifrons and Monodontomerus.

To determine the intra-nest gender distribution of immature $O$. cornifrons, 22 cardboard straw nests were gathered from a blueberry farm located in Independence, WV (N 39.469870, W -79.934519). Nests were dissected using a Dremel ${ }^{\circledR}$ tool (Robert Bosch Tool Corporation, Gerlingen, Germany) and the length of each bee cell was determined using calipers. Bee gender in each cell was determined by cutting a " $\mathrm{v}$-shaped" incision at the nipple of the cocoon, and using facial features (i.e., presence/absence of horns) and antennal length as separating features. The location of each male or female bee in the nest was plotted against cell position using nonlinear regression.

To determine the intra-nest distribution of Monodontomerus in cardboard straws, 122 Mondontomerus infested cardboard straws were taken from a blueberry farm located in Independence, WV. Cardboard straws were determined to be infested if they had Mondontomerus emergence holes. The distance of each emergence hole from the rear of the nest was recorded, and then plotted against cell position (based on average cell length) using nonlinear regression.

Inter-nest distribution of Monodontomerus in Straw Bundles. 
To determine the distribution of Monodontomerus in bundles of nest straws, $10 \times 10$ square bundles of straws containing $O$. cornifrons were placed at the West Virginia University Organic Farm (N 39.644666, W -79.937157) in Morgantown, West Virginia, and $12 \times 13$ bundles were placed at an apple orchard (N 39.397196, W -77.895350) in Kearneysville, WV. Each nest (i.e., cardboard straw) was assigned an X, Y coordinate. Monodontomerus emergence holes were recorded for each nest coordinate in an individual bundle, and then data was summed across all bundles for each nest position.

The resulting spatial data was analyzed using GS+ version 9 (Gammadesign, Plainwell, MI). The GS+ software generates a semivariogram which has several useful properties for elucidating spatial trends of $O$. cornifrons nest infestation by Monodontomerus. First, the parameters of the semivariogram include the sill (the highest level of semivariance, or asymptote, of the spatial model), nugget (the y-axis and measurement of microscale variation and error in the model), and the range (the point at which the model reaches its asymptote, which describes the distance of spatial dependency among data points). Second, the semivariogram can be used to create a map through a process known as kriging, which is a form of interpolation that may be used to predict values at unsampled locations or to explore the spatial pattern of a set of measurements.

\section{Results}

Intra-nest distribution Osmia cornifrons and Monodontomerus.

The distribution of female and male $O$. cornifrons in cardboard straws was similar to that reported previously for $O$. cornifrons in wooden observation nest blocks (McKinney and Park 2012). The distribution of female $O$. cornifrons was best described by a logarithmic distribution 
with a greater occurrence of females deeper inside cardboard straws $\left(y=-1.2806 x^{2}+22.965 x+-\right.$ 31.859, $P<0.05, r^{2}=0.8169$ ), while the distribution of male $O$. cornifrons was highest in the middle of the nest $\left(y=-31.4 \ln (x)+82.927, r^{2}=0.7936\right)$ (Figure $2 \mathrm{a}$ and $\left.\mathrm{b}\right)$. The average length of O. cornifrons nest cells was $9.13 \mathrm{~mm}$. The distribution of Monodontomerus emergence holes was best described by a fourth order polynomial distribution $\left(y=0.0084 x^{4}-0.2308 x^{3}+1.94 x^{2}-\right.$ 4.3502x $\left.+4.5385, P<0.05, r^{2}=0.9685\right)$ with the greatest occurrence of these holes being in the outermost cells of the nest (Figure 1).

\section{Inter-nest distribution of Monodontomerus in Straw Bundles.}

Anisotropic semivariograms for both Morgantown and Kearneysville showed a spatial trend in all directions (Figure 2), which is indicative of one of two spatial arrangements. In the first arrangement, a spatial distribution in which there were many wasps located centrally in cardboard straw bundles, but whose numbers gradually lowered toward the outside of the bundle, could cause the observed pattern. In the second arrangement, a spatial distribution in which a "hole" (low number of Monodontomerus) was present in the center of the cardboard straw bundle, with numbers of Monodontomerus gradually increasing towards the outside of the bundle, could also cause the pattern . Interpolation of the distribution of Monodontomerus in nest bundles revealed that the second situation was the case (Figure 3). See Table 1 for specific parameters of the anisotropic semivariograms.

\section{Discussion}

This study showed that Monodontomerus parasitism in Osmia cornifrons nests managed in cardboard straws occurs more frequently in the outermost nest cells (i.e., those near the nest 
entrance) which are typically occupied by male bees. We also showed that the outermost nests in a nest bundle is more susceptible to Monodontomerus parasitism. To the authors' knowledge, no previous studies have described the intra- and inter-nest distribution of Monodontomerus parasites. Several studies have previously discussed the distribution of male and female $O$. cornifrons in nests (Maeta 1978, McKinney and Park 2012), and our study corroborates the findings of those previous studies

Of the 25 species of New World Monodontomerus listed by Grissel (2000), eight are known from the eastern United States, and of these eight, four species have aculeate Hymenopteran hosts. Those species are M. aeneus (Fonscolmbe), M. mandibularis Gahan, M. montivagus Ashmead, and M. obscurus Westwood. Of the species listed, only M. obscurus has been recorded as a parasite of Osmia cornifrons Radozskowski, the bee host used in this study. This information leads us to believe the species in our study was M. obscurus. It is worth consideration though that $M$. aeneus, $M$. mandibularis, and $M$. montivagus are all confirmed parasites of other Osmia species (Grissel 2007).

The intra-nest distribution of Monodontomerus showed a skewed distribution with many exit holes present in the very last nest cell. There are two possible causes for this result. First, because Monodontomerus in our study area are active late in the nesting season of Osmia cornifrons, their access to Osmia prepupae at the back of the nest is restricted by nest partitions (i.e., mud walls) built by the adult host. Breaking through the cell partitions may have a high energy cost, and therefore Monodontomerus would choose to lay eggs on the closest (i.e., easiest access) Osmia prepupa available. These prepupa would be near the front of the nest. The second possible cause for the high occurrence of Monodontomerus exit holes is associated with Monodontomerus emergence. When Monodontomerus develop to adulthood in a nest cell, they 
are trapped in a cell surrounded by mud walls and nesting materials. To exit the nest they must chew through either one of these substances. Rau (1947) observed that one Monodontomerus would begin to chew through a mud wall, and others would follow through. Rau 1947 called this individual the "pioneer." It is possible that the pioneer wasp chooses to continue through mud walls until it reaches the vestibular cell (i.e., the last cell) of the nest. If this is the case it would be presented with the daunting task of chewing through the cell cap, which is much thicker than the other mud walls in the nest (McKinney and Park 2012). Given the choice between exiting through the cell cap or the cardboard nest material, the latter option may simply be the easier choice. If this second option is the case, then intra-nest distribution of Monodontomerus is being skewed as an artifact of the pioneer wasp's behavior. The distribution holds true for emergencehole locations regardless. Additionally, excluding the Monodontomerus emergence holes in the final cells of the Osmia cornifrons nests used in this study would still leave the same interpretation regarding the spatial pattern of Monodontomerus, only it would be less exaggerated.

The spatial pattern of Monodontomerus was the same for both $10 \times 10$ and $12 \times 13$ bundles of cardboard straw nests at two study sites. In both cases, the trend revealed higher probabilities of Monodontomerus presence in nests located on the perimeter of the bundles. The corners of the bundles, in particular, showed high numbers of Monodontomerus infestation. These results may be caused by one of two possibilities. First, Osmia cornifrons may have a preference to nest in straws on the outside of a bundle (presumably to make nest location between foraging trips easier). If this is the case, Monodontomerus must go where its host is, so the distribution of Osmia cornifrons would dictate the distribution of Monodontomerus. The second possibility is that because nests on the outside of a bundle are more exposed (especially 
the corner nests), those nests are easier for Monodontomerus to locate. Because

Monodontomerus locates hosts using volatile cues (Fillela et al. 2011) rather than echolocation (i.e., vibrational sounding [Broad and Quicke 2000]), the latter is likely the case. In a nest bundle populated with many Osmia cornifrons nests, there would likely be overwhelming volatile signals at the nest site, especially in the middle of the bundle. The volatile cloud should be smaller at the edge of the nest, making host location easier for Monodontomerus.

These described spatial patterns have important implications for the management of Osmia cornifrons for pollination. McKinney and Park (2013) described the spatial distribution of another Osmia cornifrons pest, Chaetodactylus krombeini (Acari: Chaetodactylidae). That parasite was shown to have an intra-nest spatial trend toward the rearmost cells of the nest, where most female Osmia are found. There are many more male than female bees in $O$. cornifrons populations (White et al. 2009), and the number of females are likely an important limiting factor in annual population growth. Additionally, female bees are responsible for the majority of pollination that occurs. For these reasons Chaetodactylus may be considered a more important pest to manage than Monodontomerus.

We found that nests on the outside of a bundle of nests were the most susceptible to Monodontomerus parasitism, and that within a nest, those cells closest to the entrance had the highest number of Monodontomerus emergence holes. Further investigation on the emergence and host location strategies of Monodontomerus is needed to determine the definitive reason for these spatial trends. Traditional management techniques for Monodontomerus such as using wooden nesting blocks as opposed to cardboard straws, and removing bees from the field after nesting, are advisable. A method of masking the volatile signal used by Monodontomerus for host location may prove useful in reducing wasp populations. 


\section{Acknowledgements}

The author would like to thank Vicki Kondo and Jacob Goldner for their assistance in gathering data for this project, and the researchers of the WVU Tree Fruit Research and Education Center for their assistance in managing the Kearneysville, WV Osmia cornifrons population.

\section{References Cited}

Abel, C.A., R. L. Wilson, R. L. Luhman. 2003. Pollinating efficacy of Osmia cornifrons and Osmia lignaria, subsp. lignaria (Hymenoptera: Megachilidae) on three Brassicaceae species grown under field cages. J. Entomol. Sci. 38(4): 545-552.

Bosch, J., and W. P. Kemp. 2001. How to manage the blue orchard bee, Osmia lignaria, as an orchard pollinator. Washington, DC, Sustainable Agriculture Networks.

Broad, G. R., and D. L. J. Quicke. 2000. The adaptive significance of host location by vibrational sounding in parasitoid wasps. Proc. Biol. Sci. 267(1460): 2403-2409.

Digweed, S. C., C. J. K. MacQuarrie, D. W. Langor, D. J. M. Williams, J. R. Spence, K. L. Nystrom, and L. Morneau. 2009. Current status of invasive alien birch-leafmining sawflies (Hymenoptera: Tenthredinidae) in Canada, with keys to species. Can. Entomol. 141: 201-235.

Eves, J.D. 1970. Biology of Monodontomerus obscurus Westwood, a parasite of the alfalfa leafcutting bee, Megachile rotundata (Fabricius) (Hymenoptera: Torymidae; Megachilidae). Melandaria 4: 1-18.

Fillela, I., J. Bosch, J. Llusià, R. Seco, and J. Peñuelas. 2011. The role of frass and coccoon volatiles in host location by Monodontomerus aeneus, a parasitoid of megachilid solitary bees. Environ. Entomol. 40(1): 126-131. 
Gahan, A.B. 1941. A revision of the chalcid-flies of the genus Monodontomerus in the United States National Museum. Proc. U. S. Natl. Mus. 90: 461-482.

Goodpasture, C. 1975. Comparative courtship behavior and karyology in Monodontomerus (Hymenoptera: Torymidae). Ann. Entomol. Soc. Am. 68(3): 391-397.

Grissel, E.E. 2000. A revision of the New World Monodontomerus (Hymenoptera: Chalcidoidea: Torymidae). Contrib. Am. Entomol. Inst. 32(1): 1-90.

Grissel, E.E. 2007. Torymidae (Hymenoptera: Chalcidoideae) associated with bees (Apoidea), with a list of chalcidoid bee parasitoids. J. Hymenopt. Res. 16(2): 234-265

Hobbs, G.A., and M. D. Krunic. 1971. Comparative behavior of three chalcidoid (Hymenoptera) parasites of the alfalfa leafcutter bee, Megachile rotundata, in the laboratory. Can. Entomol. 103: 674-685.

Howard, L. O., and W. F. Fiske. 1911. The importation into the United States of the parasites of the gypsy moth and the browntail moth. U. S. Dep. Agric. Bur. Entomol. Bull 91.

Krunić, M., L. Stanisavljević, M. Pinzauti, and A. Felicioli. 2005. The accompanying fauna of Osmia cornuta and Osmia rufa and effective measures of protection. Bull. Insectol. 58(2): 141-152.

Lee, S. -B., D. -K. Seo, K. -H. Choi, S. -W. Lee, H. -J. Yoon, H. -C. Park, Y. -D. Lee. 2008. The visited insects on apple flowers, and the characteristics on pollinating activity of pollinators released for pollination of apple orchards. Kor. J. Apicult. 23(4): 275-282.

Macivor, J.S., and B. Salehi. 2014. Bees species-specific nesting material attracts a generalist parasitoid: Implications for co-occurring bees in nest box enhancements. Chem. Ecol. 43(4): 1027- 1033. 
McGugan, B. M., and H. C. Coppel. 1962. Biological control of forest insects - 1910-1958. In: A Review of the Biological Control Attempts Against Insects and Weeds in Canada. Commonwealth Agricultural Bureaux Franham Royal, UK, pp. 35-127.

McKinney, M.I., and Y. -L. Park. 2013. Distribution of Chaetodactylus krombeini (Acari: Chaetodactylidae) within Osmia cornifrons (Hymenoptera: Megachilidae) nests: implications for population management. Exp. Appl. Acarol. 60(2): 153-161.

Parker, F. D., and P. F. Torchio. 1980. Management of wild bees, pp. 144-160. Beekeeping in the United States. United States Department of Agriculture. Handbook 335: 1-193.

Rau, P. 1947. Bionomic and Monodontomerus mandibularis Gahan, with notes on other chalcids of the same genus. Ann. Entomol. Soc. Am. XL: 221-226.

Van Driesche, R. G., R. I. Carruthers, T. Center, M. S. Hoddle, J. Hough-Goldstein, L. Morin, L. Smith, D. L. Wagner, B. Blossey, V. Brancatini, R. Casagrande, C. E. Causton, J. A. Coetzee, J. Cuda, J. Ding, S. V. Fowler, J. H. Frank, R. Fuester, J. Goolsby, M. Grodowitz, T. A. Heard, M. P. Hill, J. H. Hoffmann, J. Huber, M. Julien, M. T. K. Kairo, M. Kenis, P. Mason, J. Medal, R. Messing, R. Miller, A. Moore, P. Neuenschwander, R. Newman, H. Norambuena, W. A. Palmer, R. Pemberton, A. Perez Panduro, P. D. Pratt, M. Rayamajhi, S. Salom, D. Sands, S. Schooler, M. Schwarzländer, A. Sheppard, R. Shaw, P. W. Tipping, R. D. van Klinken. 2010. Classical biological control for the protection of agricultural ecosystems. Biol. Contr. 54: S2-S33.

Washburn, J.O. 1984. The gypsy moth and its parasites in North America: A community in equilibrium? Am. Nat. 124(2): 288-292.

White, J., Y. Son, and Y-L. Park. 2009. Temperature-dependent emergence of Osmia cornifrons (Hymenoptera: Megachilidae) adults. J. Econ. Entomol. 102(6): 2026-2032. 
Table 1. Anisotropic semivariogram parameters for the distribution of Monodontomerus spp. emergence holes in Osmia cornifrons nest bundles.

\begin{tabular}{lllllll}
\hline Site & Direction & Model & $\mathbf{R}^{2}$ & Nugget & Range & Sill \\
\hline Morgantown & $134^{\circ}$ & $\mathrm{y}=0.1382 \mathrm{x}+2.4504$ & 0.9424 & 2.450 & $-*$ & $-*$ \\
& $179^{\circ}$ & $\mathrm{y}=0.3538 \mathrm{x}+2.0511$ & 0.9919 & 2.051 & - & - \\
& $224^{\circ}$ & $\mathrm{y}=0.4743 \mathrm{x}+2.7218$ & 0.9516 & 2.722 & - & - \\
& $269^{\circ}$ & $\mathrm{y}=0.3293 \mathrm{x}+2.3613$ & 0.9795 & 2.361 & - & - \\
\hline Kearneysville & $76^{\circ}$ & $\mathrm{y}=0.3592 \mathrm{x}+8.6845$ & 0.4052 & 8.684 & - & - \\
& $121^{\circ}$ & $\mathrm{y}=1.6546 \mathrm{x}+7.7005$ & 0.9676 & 7.700 & - & - \\
& $166^{\circ}$ & $\mathrm{y}=1.8036 \mathrm{x}+6.9815$ & 0.9787 & 6.981 & - & - \\
& & & & & & \\
& $211^{\circ}$ & $\mathrm{y}=0.6398 \mathrm{x}+9.0719$ & 0.7132 & 9.072 & -
\end{tabular}

* Range and sill are not applicable to linear semivariograms. 

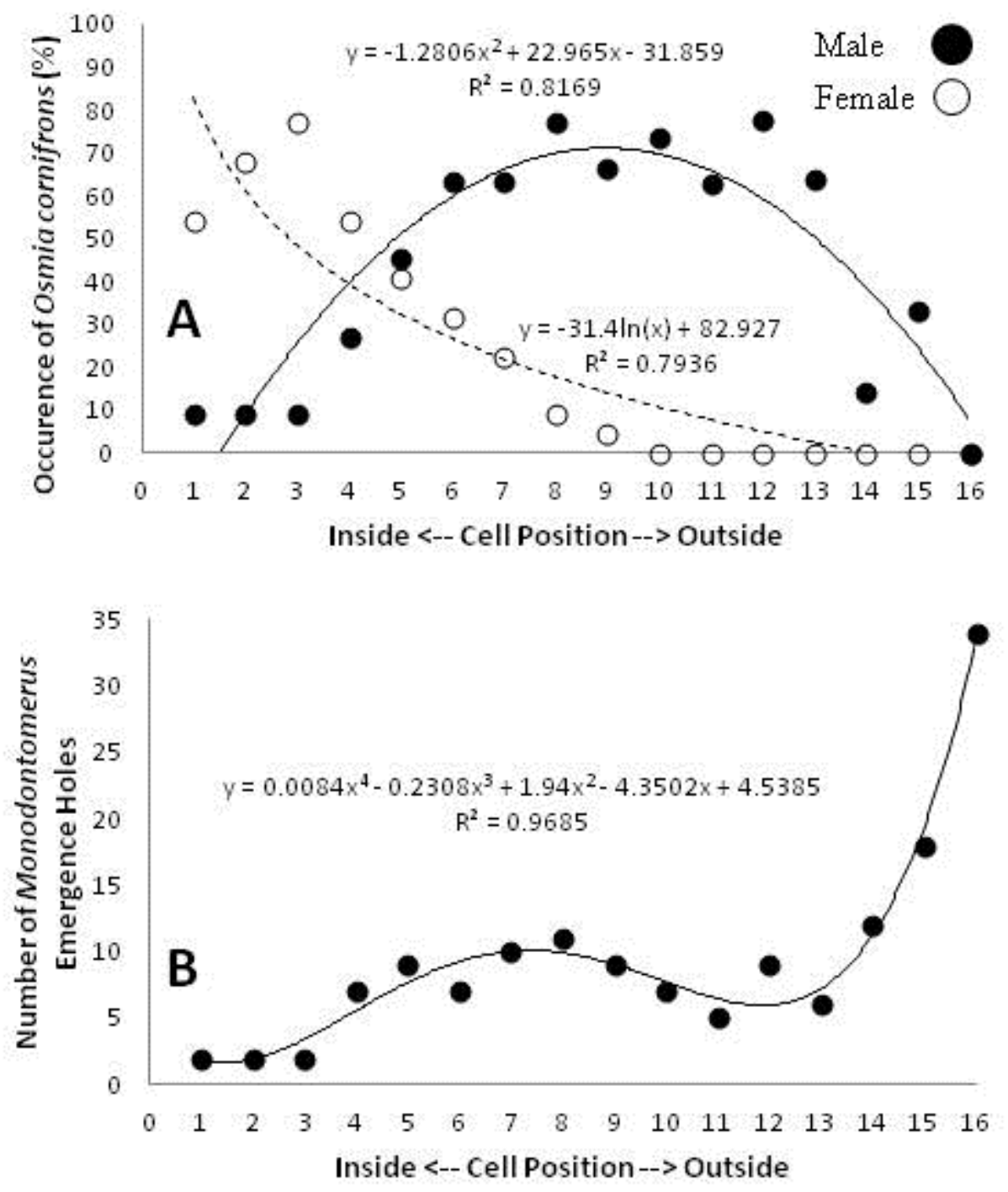

Figure 1. Distribution of Osmia cornifrons (A) and Monodontomerus (B) shows male O. Cornifrons may be more susceptible than females to wasp parasitism. 

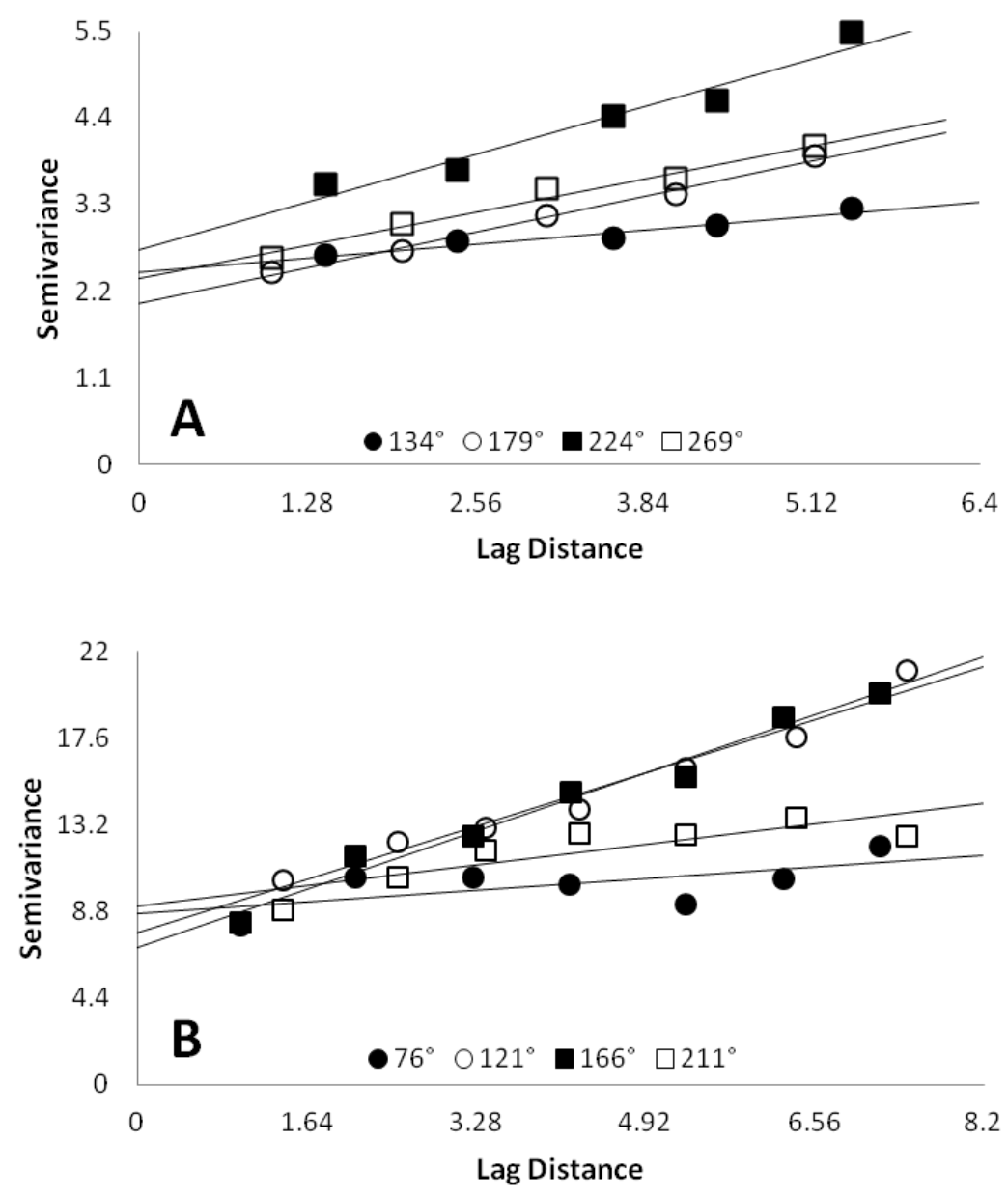

Figure 2. Anisotropic semivariograms for Morgantown (A) and Kearneysville (B) showing the linear relationship between semivariance and lag distance of Monodontomerus parasitism in cardboard straw bundles. 


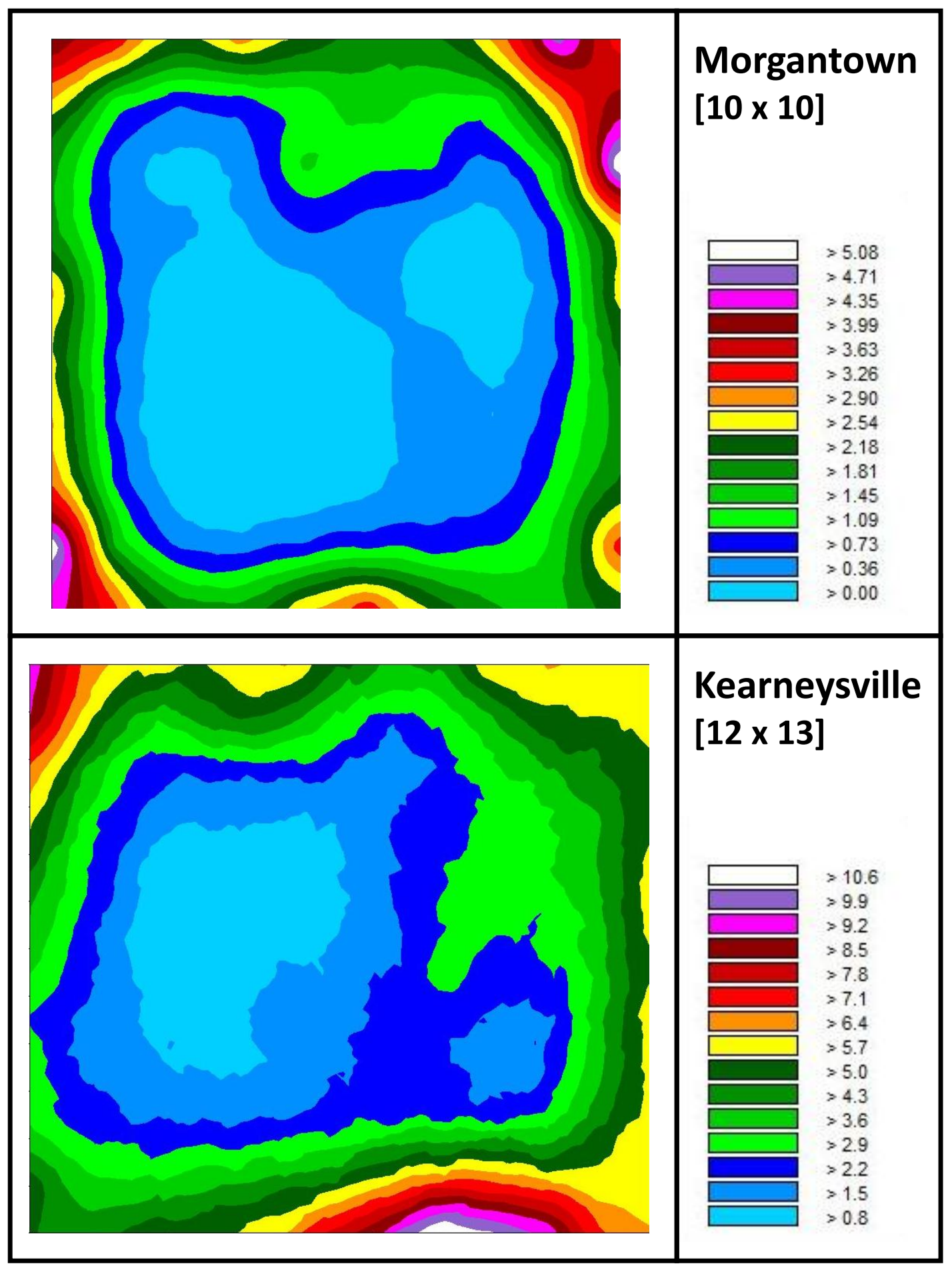

Figure 3. Interpolation of Monodontomerus spp. emergence holes in nests of Osmia cornifrons generated by using kriging. 


\section{CHAPTER 6: CONCLUSIONS}

This dissertation aimed to describe the bee fauna of West Virginia, and to aid of bee monitoring, conservation, and management practices in West Virginia. Chapter 1 served as the first compiled checklist of the state's bees, complete with valuable life history and collection information. It is my hope that chapter 1 will serve not as a definitive list of West Virginia's bee fauna, but as a starting point for future conservation and bee monitoring work. Chapter 2 furthers this goal by enhancing bee monitoring sampling plans. Through a geostatistical approach, I was able to determine the distance at which pan traps used for collecting bees would gather samples independently from one-another. This will certainly be key information in bee monitoring programs, not only in West Virginia, but anywhere that a bee monitoring program is desired. In chapter 4 I determined the thermal window of Osmia cornifrons and developed models of the bee's development using both time and temperature as predictors. This work provides key parameter values for predicting the effects of climate change on bees, and serves as a model for bee conservation efforts when temperature is an important factor. Chapter 5 described the spatial distribution of the parasitic wasp Monodontomerus within O. cornifrons linear nests and within bundles of nests. Because it is common for linear, tube-shaped nests to be used to attract native bees to small agricultural lands, flower gardens, and similar areas, it is desirable to understand how common parasites may interact with bees utilizing these nests (i.e., Megachilidae). This work will be useful in protecting megachilid bees from parasites in areas where pollination is needed, but pollinators are scarce.

Monitoring, conservation, and management of bees in West Virginia is still greatly understudied. There have been few previous entomologists in the state who published works related to these endeavors, perhaps because historically the importance of native (non-Apis) bees 
was not fully realized, and because Apis mellifera was not being placed under such great strain to provide our agricultural pollination needs. Future work in the state should focus on continuous monitoring of the states bee fauna, with special effort given to those understudied areas determined in chapter 2. Additionally, temperature-dependent modelling for bees outside of the Megachilidae (and their host plants) would be beneficial for developing holistic models of climate changes impact on pollinators in our region. Finally, additional work on bee parasite-host interactions for abundant taxa may make management of non-Apis bees for conservation and pollination more feasible.

If I could make a single suggestion that I feel would have the greatest impact on the conservation of native bees and agricultural production here in the United States, my suggestion would be to develop a fully standardized bee monitoring program at the national scale on a tenyear cycle. That is to say, sampling would occur over ten-year periods and reports would be generated at the end of each ten-year cycle. These reports would be given to a national council responsible for compiling the information and comparing data from each cycle with that of the previous cycle. Standardized methods would permit comparisons of faunal data over space and time and detection of shifts in species abundance, evenness, and other population indices. By utilizing a ten-year cycle the national monitoring program could act as a census of our pollinator fauna and aid in the detection of invasive pollinators. Being vigilante in the detection of pollinator related issues is the only way to ensure we conserve this valuable national asset. It is my hope that this small contribution to our understanding of West Virginia's bees will inspire greater interest in the monitoring and conservation of our native bees at the national scale. 